\section{Arkivoc

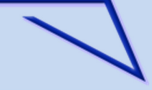

Archive for

Organic Chemistry
The Free Internet Journal

for Organic Chemistry
Review

Arkivoc 2018, part i, 0-0

\title{
2-Chloroquinoline-3-carbaldehydes: synthesis and reactions (2012-2017)
}

\section{Mohammed A. M. Massoud ${ }^{a}$, Waleed A. Bayoumi $^{\mathrm{a}, \mathrm{b}}$, Abdelbasset A. Farahat ${ }^{\mathrm{a}}$, Magda A. El-Sayed ${ }^{\mathrm{a}, \mathrm{c}}$, and Basem Mansour ${ }^{\text {b }}$}

${ }^{a}$ Pharmaceutical Organic Chemistry Dept., Faculty of Pharmacy, Mansoura University, Mansoura 35516, Egypt

${ }^{b}$ Pharmaceutical Chemistry Dept., Faculty of Pharmacy, Delta University for Science and Technology, Gamasa, Egypt

'Pharmaceutical Chemistry Dept., Faculty of Pharmacy, Horus University, New Damietta, Egypt Email: massoudmam@yahoo.fr

\section{Abstract}

This review discuss in details the synthesis and reactions of 2-chloroquinoline-3-carbaldehydes during years of 2012-2017. The reactions are subdivided into groups, according to type of reaction, including reactions of both chloro and/or aldehyde substituents. Most applied reactions have been successfully utilized for synthesis of different biologically and pharmacologically active derivatives.

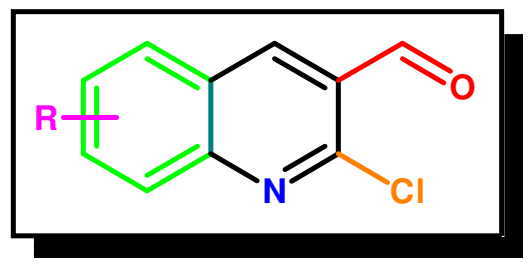

Keywords: Aldehydes, aromatic nucleophilic substitution, 2-chloroquinoline-3-carbaldehydes, cyclization, quinolines, Vilsmeier-Haack reaction 


\section{Table of Contents}

1. Introduction

2. Synthetic Methods

2.1. The classical Vilsmeier-Haack reaction

2.2. Oxidation of the corresponding alcohol

3. Chemical Reactions

\subsection{Cyclization reactions}

3.1.1. Cyclization at both aldehyde and chloro groups

3.1.2. Cyclization at aldehyde group

3.1.3. Cyclization via multicomponent reaction

3.1.3.1. Cyclization via three component reaction

3.1.3.2. Cyclization via four component reaction

3.2. Reduction of the aldehyde group

3.3. Oxidation of the aldehyde group

3.4. Condensation reactions

3.4.1. Reactions with active methylene compounds

3.4.2. Reactions with hydrazine, hydroxylamine, hydrazides, (thio)semicarbazide, and urea

3.4.3. Reactions with amines and amides

3.4.4. Miscellaneous reactions

4. Conclusions

References

\section{Introduction}

In the recent years, the chemistry of 2-chloroquinoline-3-carbaldehydes has received considerable attention owing to their synthetic and reactions versatility, in addition to a wide variety of biological activity. ${ }^{1}$ These aldehydes were also used as synthetic intermediates for the preparation of large numbers of heterocyclic systems ${ }^{2}$ and stereoselective ligands $s^{3,4}$ of various importance.

Polyfunctionalized heterocyclic compounds play important roles in the drug discovery and drug analysis processes; about $68 \%$ of available market drugs are containing heterocyclic ring system. ${ }^{5}$ Hence it is not surprising that research on the synthesis of polyfunctionalized heterocyclic compounds has received significant attention. The quinolone or 1-azanaphthalene ring system represent a wide occurrence in the nature as substituted and fused ring derivatives, Its derivatives have been known to display a wide range of pharmacological activities such as antimalarial ${ }^{6}$, anti-bacterial ${ }^{7}$, anticancer ${ }^{8}$, antifungal ${ }^{9}$, anthelmintic ${ }^{10}$, cardiotonic $^{11}$, anticonvulsant and antihypertensive ${ }^{12}$, anti-inflammatory and analgesic activity. ${ }^{13}$ In addition, Quinoline has a privileged scaffold in cancer drug discovery. ${ }^{14}$ 2-Chloroquinoline-3-carbaldehydes has been reviewed during the period from 1979 to $1999^{15}$ and from 1999 to $2011^{16}$, Herein, in this review, we cover the versatile synthetic methods and reactions from 2012 until 2017.

\section{Synthetic Methods}

Two important general procedures are used for synthesis of 2-chloroquinoline-3-carbaldehyde and its derivatives $\mathbf{I}$. 


\subsection{The classical Vilsmeier-Haack reaction}

It is the most convenient and traditional route for the synthesis of 2-chloroquinoline-3-carbaldehydes. This strategy consists of a multicomponent reaction that involves processes of chlorination, formylation and cyclization of acetanilides by the action of the Vilsmeier's reagent $\mathrm{DMF} / \mathrm{POCl}_{3}$ to afford 2-chloroquinoline-3carbaldehydes I (Scheme 1). ${ }^{17,18}$

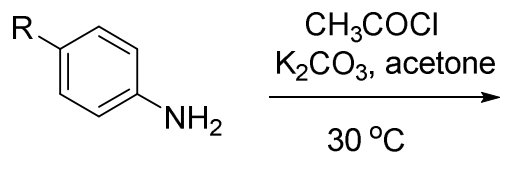

1a-c
(2)

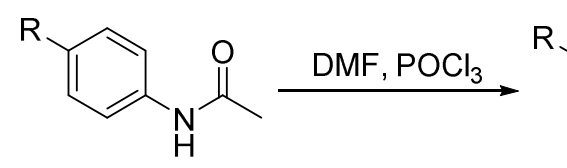

2a-c

a: $\mathrm{H}, \mathbf{b}: \mathrm{Me}, \mathbf{c}: \mathrm{MeO}$<smiles>[R]c1ccc2nc(Cl)c(C=O)cc2c1</smiles>

I

\section{Scheme 1}

Vilsmeier formylation of acetamide derivatives 4a-j afforded the corresponding 2-chloroquinoline-3carbaldehydes I, which upon treatment with $\mathrm{CH}_{3} \mathrm{COCl}$ and triethyl amine in DMF, furnished the respective 4oxazetidin-1-yl derivatives I' (Scheme 2). ${ }^{19}$

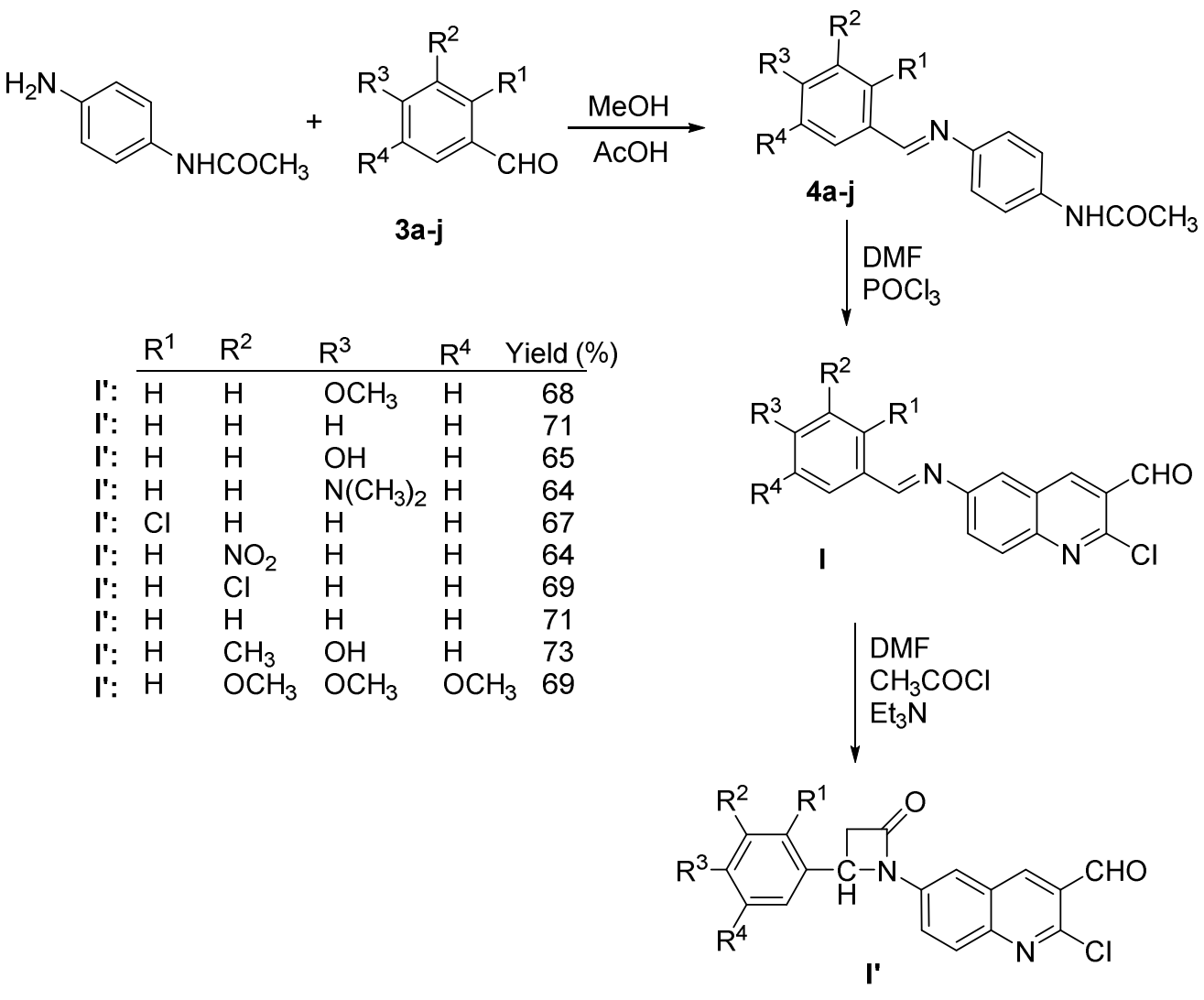

\section{Scheme 2}

An alternative, convenient and efficient procedure for the synthesis of 2-chloroquinoline-3carbaldehydes I was carried out by the action of Vilsmeier's reagent on acetanilides $5 a-i$ using $\mathrm{PCl}_{5}$ as chlorinating agent in place of $\mathrm{POCl}_{3}$ was developed (Scheme 3). ${ }^{20}$ 
<smiles>[R]=CC=C(C)NC(C)=O</smiles>

5a-i

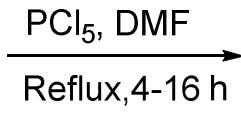<smiles>O=Cc1cc2cc[R1]cc2nc1Cl</smiles>

I
I: $\frac{\mathrm{R} \quad \text { Yield (\%) }}{\mathrm{H} \quad 78}$

I: $6-\mathrm{CH}_{3} \quad 74$

I: $7-\mathrm{CH}_{3} \quad 66$

I: $8-\mathrm{CH}_{3} \quad 67$

I: $6-\mathrm{OCH}_{3} \quad 54$

I: $7-\mathrm{OCH}_{3} \quad 65$

I: $6-\mathrm{Br} \quad 30$

I: $7-\mathrm{Cl} \quad 35$

I: $6-\mathrm{NO}_{2} \quad 0$

\section{Scheme 3}

\subsection{Oxidation of the corresponding alcohol}

2-Chloroquinoline-3-carbaldehyde I was obtained by oxidizing its corresponding alcohol 6 using diethyldiazene-1,2-dicarboxylate (DEAD) and catalytic $\mathrm{ZnBr}_{2}$ in refluxing toluene (Scheme 4). ${ }^{21}$<smiles>OCc1cc2ccccc2nc1Cl</smiles>

6

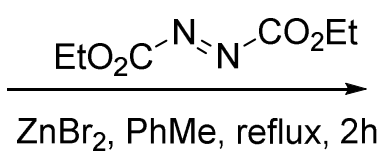

$\mathrm{ZnBr}_{2}, \mathrm{PhMe}$, reflux, $2 \mathrm{~h}$<smiles>O=Cc1cc2ccccc2nc1Cl</smiles>

I, $86 \%$

\section{Scheme 4}

\section{Chemical Reactions}

\subsection{Cyclization reactions}

3.1.1. Cyclization at both aldehyde and chloro groups. A mixture of 2-chloroquinoline-3-carbaldehyde I and heterocyclic ketene $7 \mathrm{a}-\mathrm{o}$ was stirred for $20 \mathrm{~h}$ at $75^{\circ} \mathrm{C}$ in 1,4-dioxane and in presence of catalytic piperidine to afford of 1,3-diazaheterocycle fused naphthyridine derivatives $8 a-y$, regioselectively (Scheme 5). ${ }^{22}$

A series of pyrazolo[3,4-b]quinolines 10a-h was synthesized using one-pot water mediated synthetic route under microwave irradiation involving the condensation of 2-chloroquinoline-3-carbaldehydes I with semicarbazide or 2,4-dinitrophenyl hydrazine (Scheme 6). ${ }^{23}$ 


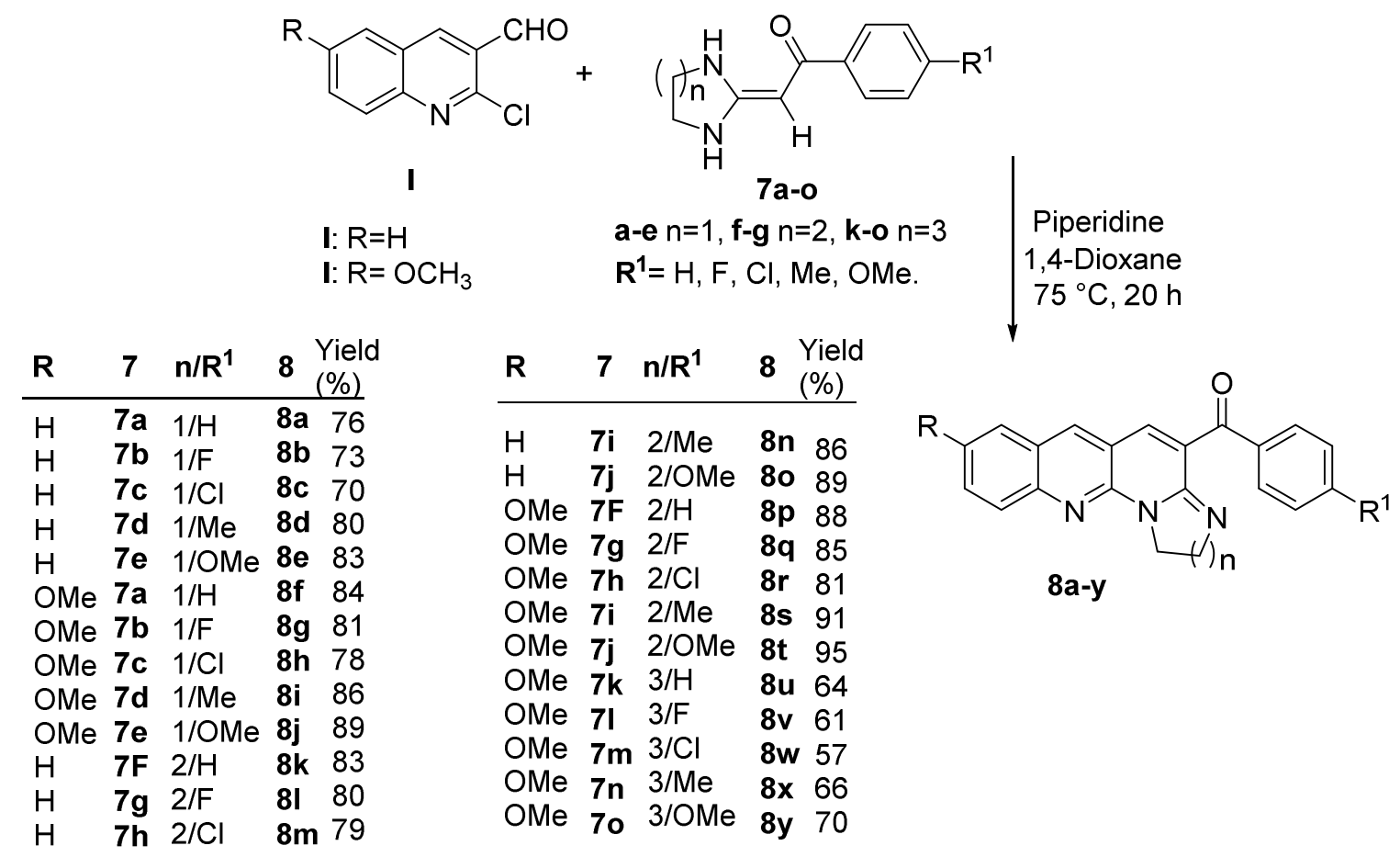

\section{Scheme 5}<smiles>[R][X]1cccc(C(C)=O)c1</smiles><smiles>[R][X]1ccccc1C(C)=NO</smiles><smiles>[CH-]=C</smiles><smiles>[R]c1ccc2nc(Cl)c(C=O)cc2c1</smiles>

I<smiles>[3H]C#[13CH]</smiles><smiles></smiles>

$\mathrm{R}$<smiles>NC(=O)n1ncc2cc3cc(I)ccc3nc21</smiles>

10a-d $92-96 \%$

Reagents and Condition: i. Sodium acetate, Hydroxylamine, $\mathrm{HCl}$; ii.DMF, $\mathrm{POCl}_{3}$; iii. Semicarbazide, water, MW -1000 W; iv. 2,4-dinitrophenylhydrazine, water, MW -1000 W.

\section{Scheme 6}

Both thiopyrano[2,3-b]quinoline-3-carbaldehydes $13 \mathbf{a}$ and $13 \mathbf{b}$ were obtained as new Domino Knoevenagel/hetero-Diels-Alder (dienophile-tethered-aldehyde substrates) in $73 \%$ and $81 \%$ yields, respectively. The reaction proceeds through thia-Michael-Aldol reaction of the corresponding 2mercaptoquinoline-3-carbaldehydes $\mathbf{1 1 a}$ and $\mathbf{1 1 b}$ with 12 in ethylenediamine diacetate (EDDA) in refluxing toluene (Scheme 7). ${ }^{24-29}$ 


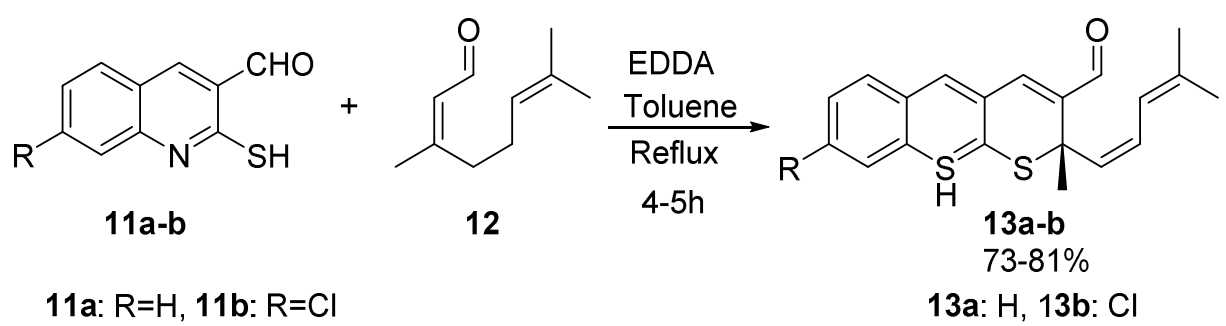

\section{Scheme 7}

2-Alkenylthiopyranoquinoline-3-carbaldehydes $13 a-b$ with heterocyclic mono- or diketones in tetrabutylammonium hydrogensulfate, under solvent-free conditions, afforded a new class of thiochromenoquinoline-fused heterocycles in good yields. The reaction is highly diasteroselective (Scheme $8) .^{30}$

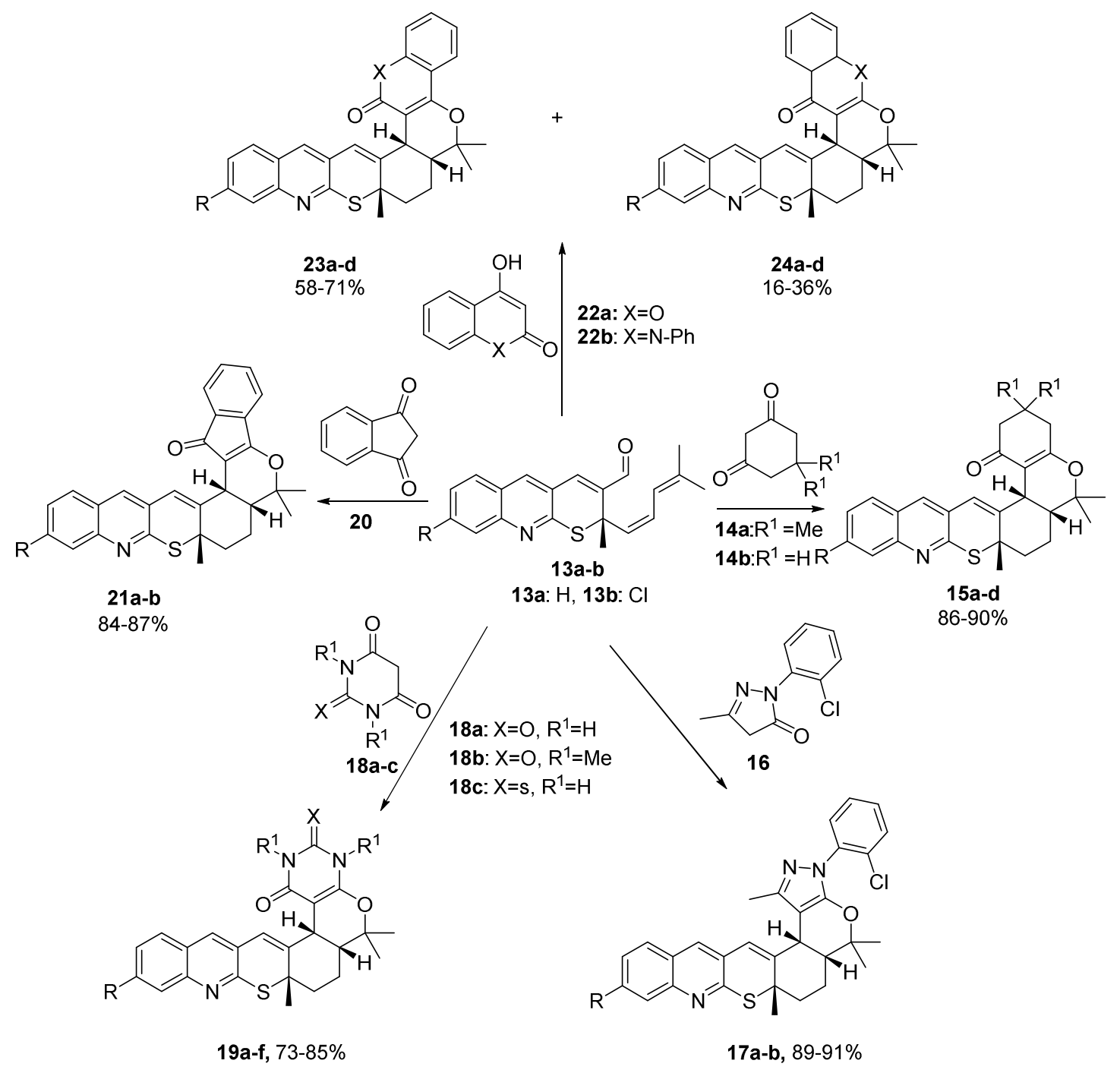

\section{Scheme 8}


3.1.2. Cyclization at aldehyde group. A new series of quinoline-based azetidinones 26a-I and thiazolidinone 27a-I analogs was developed by a simple and efficient synthetic protocol. The thione nucleus was obtained from 2-chloroquinoline-3-carbaldehyde I using sodium sulphide in DMF followed by reaction with various substituted amine to form the corresponding Schiff base intermediates 25a-i. Attempt has been made to derive final azetidinone and thiazolidinone analogs from Schiff's bases by using chloroacetyl chloride and 2mercaptoacetic acid, respectively (Scheme 9). ${ }^{31}$
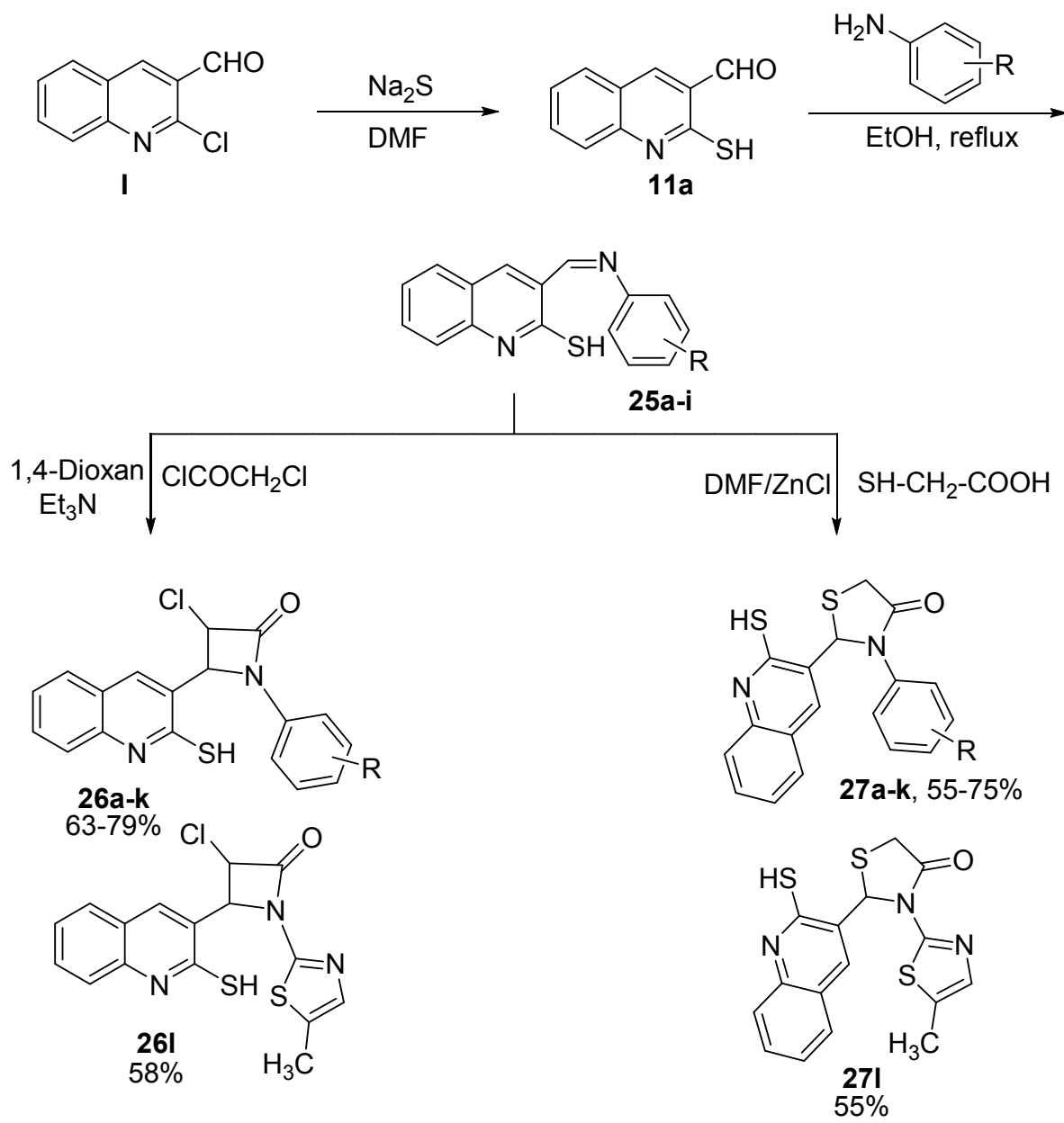

Coupled (Ar. amines)

$\mathrm{R}$ (substituted aniline)

a:H

b: $2-\mathrm{Me}$

c: $3-\mathrm{Me}$

d: $4-\mathrm{Me}$

e: 2 -nitro,

f: 3-nitro

g: 4-nitro

h: $2-\mathrm{Cl}$

i: $3-\mathrm{Cl}$

j: $4-\mathrm{Cl}$

k: 4-F

or

I: 2-Amino 5-methyl

thaizole

\section{Scheme 9}

Efficient synthesis of 3-(6'-amidoindazoloquinoline-2-(1H)-ones 29a-e was reported via addition of $1 \mathrm{H}$ Indazole-6-amine to a solution of the corresponding acid chlorides 28a-e in dry benzene in presence of few drops of pyridine under reflux. Benzo[b]-1H-indazolo[6,7- $h][1,6]$ naphthyridin-7-(6H)-ones 30a-e were prepared by heating $29 a-e$ in polyphosphoric acid (PPA) at $150{ }^{\circ} \mathrm{C}$ for $5 \mathrm{~h}$ (Scheme 10). ${ }^{32}$ 


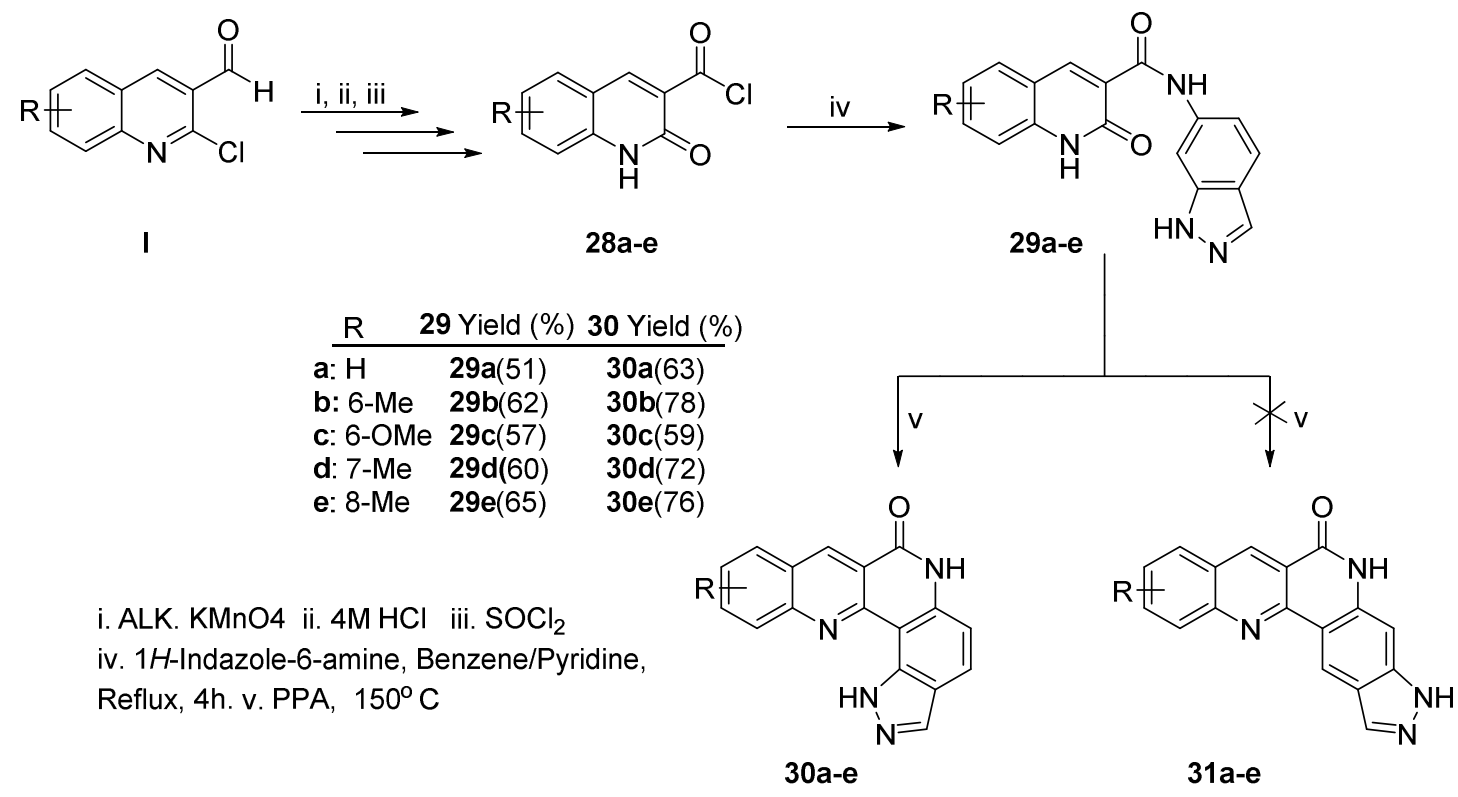

\section{Scheme 10}

The parent epoxide 3-(2-chloroquinolin-3-yl)oxiran-2-yl)(phenyl)methanone 33a and ten of its substituted derivatives 33b-k were synthesized via Darzens reaction of 2-chloroquinoline-3-carbaldehydes and its 6-substituted derivatives I with phenacyl bromide and its 2- and 4-mono- and 2,4-disubstituted derivatives 32a-c. (Scheme 11). ${ }^{33}$

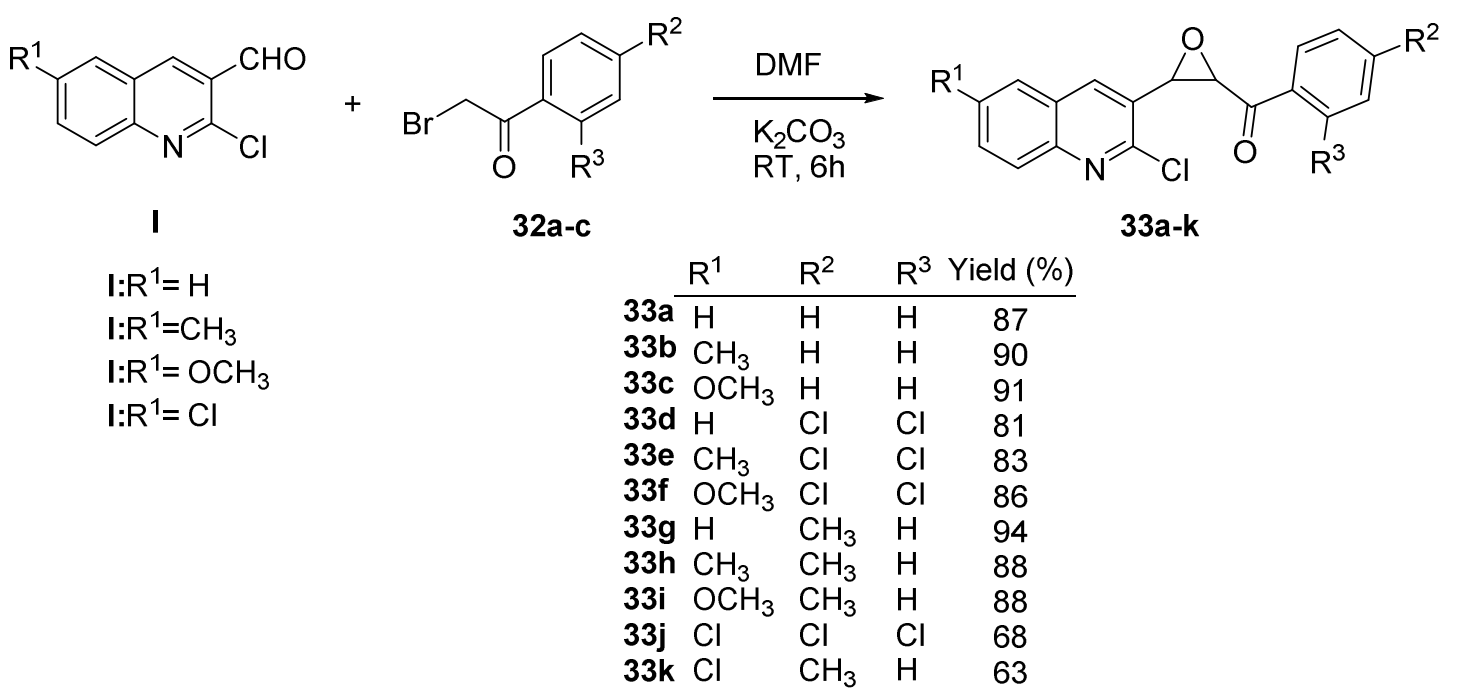

\section{Scheme 11}

3.1.3. Cyclization via multicomponent reactions (MCRs). 3.1.3.1. Three component reactions. A facile and efficient one-pot procedure for the preparation of functionalized benzo[b][1,8]naphthyridine $\mathbf{3 6}\{\mathbf{1 , 1} \mathbf{1}\}$ by three-component reaction of 2-chloroquinoline-3-carbaldehyde I(1), 1,3-dicarbonyl compound (4hydroxycoumarin) $\mathbf{3 4 ( 1 )}$, and enaminone $\mathbf{3 5 ( 1 )}$ catalyzed by L-proline was described (Scheme 12). ${ }^{34}$ 


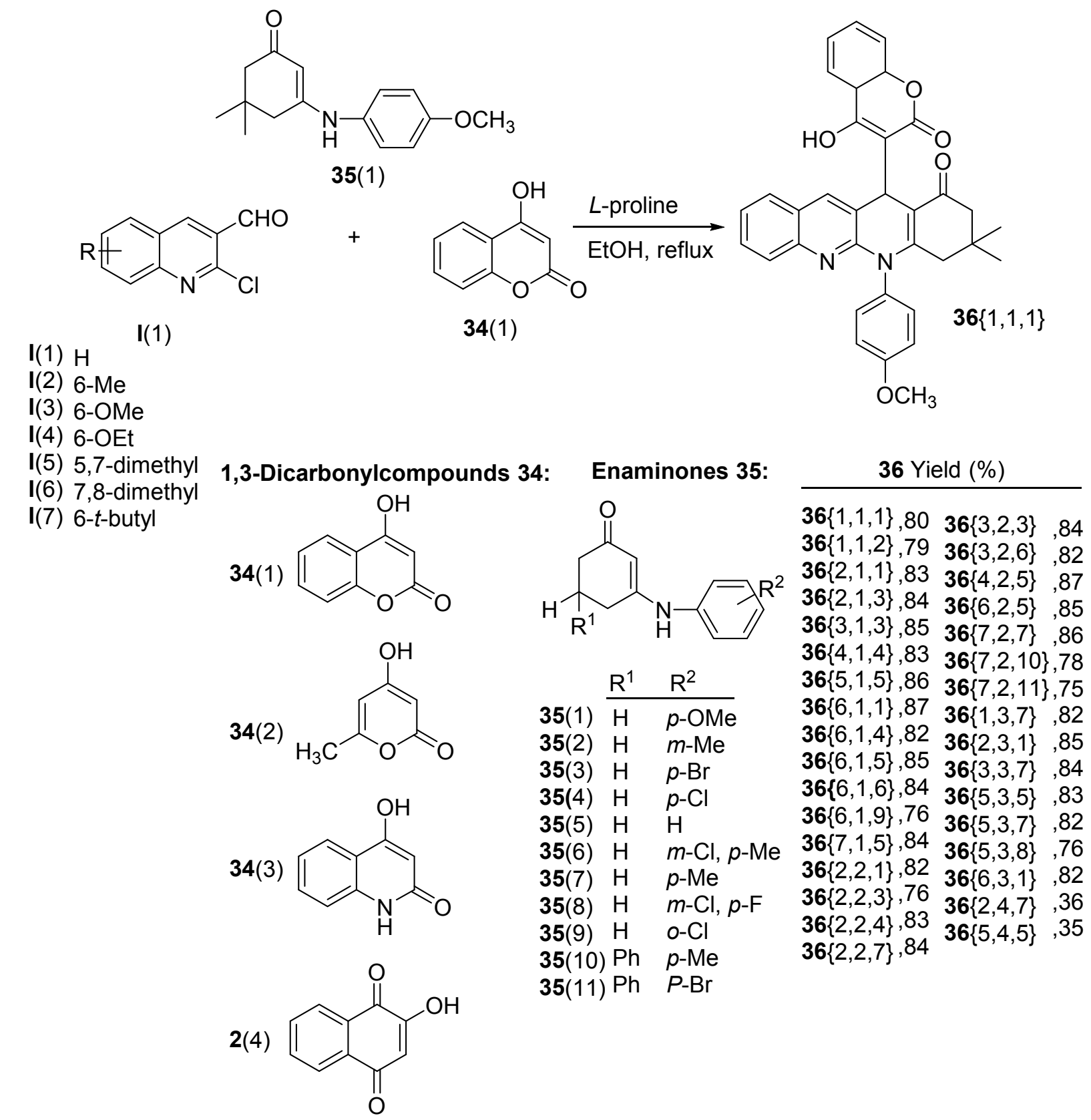

\section{Scheme 12}

The aforementioned procedure was further applied via one-pot reaction of 2-chloroquinoline-3carbaldehydes I, 6-aminouracils 38 and dimedone 37. Interestingly, the 6-Me and 6-OMe substituted quinoline aldehydes gave rise to products $40 a-c$ proceeding through intramolecular nucleophilic attack by nitrogen, while the other aldehydes gave products 39a-d resulting from attack by oxygen. The exact reason for this selectivity is at present unclear (Scheme 13). ${ }^{35}$ 


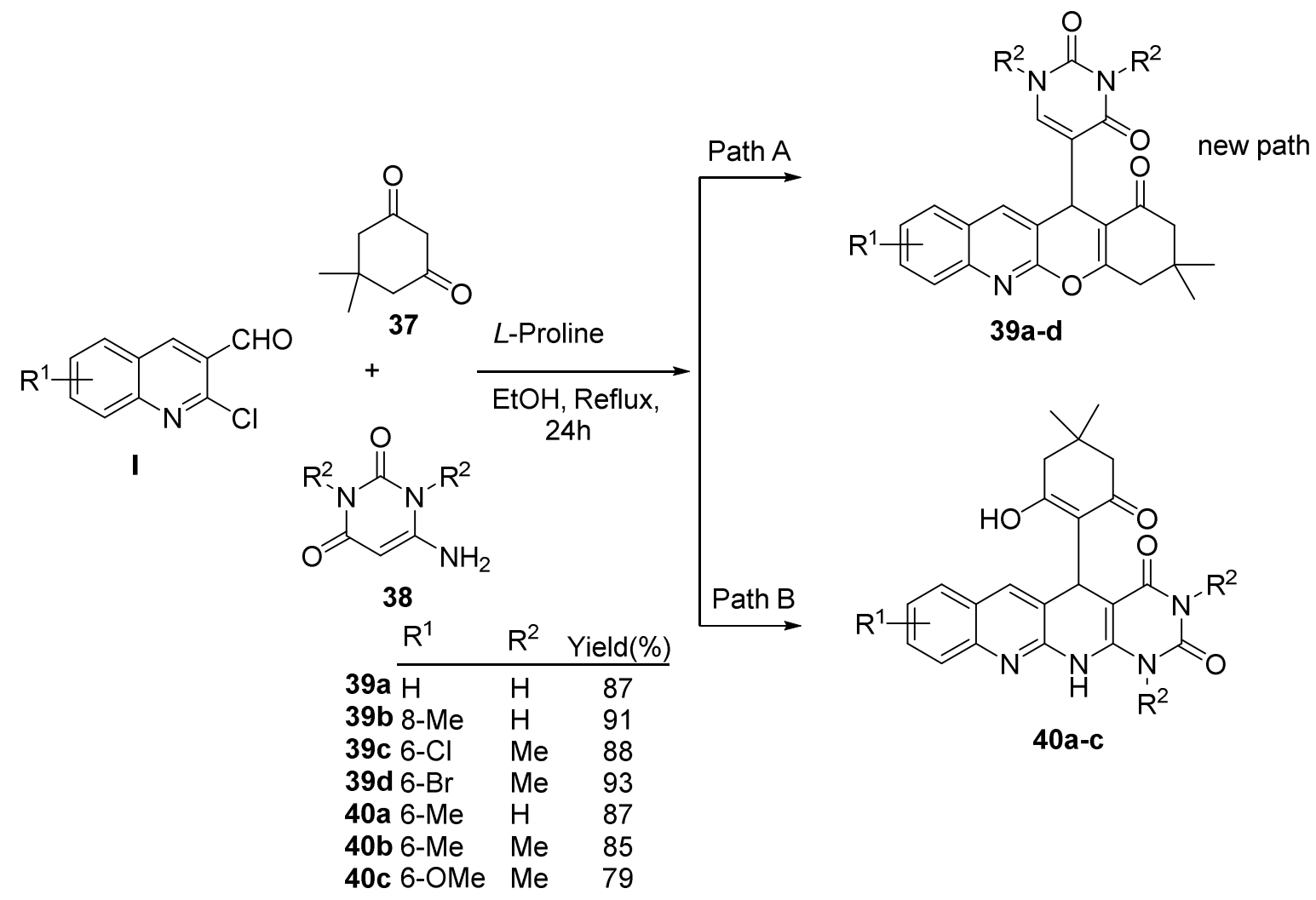

\section{Scheme 13}

An environmentally benign strategy to the synthesis of 2-amino-3-cyano 4- $H^{-c h r o m e n e s ~} 42 a-d$, via one-pot reaction involving malononitrile, various $\alpha$ - or 6 -naphthol and aldehydes $\mathbf{I}$ in the presence of morpholine in water was developed. It was found that employing this approach, aromatic aldehydes bearing electron-withdrawing groups gave higher yields of the corresponding products in shorter reaction times. (Scheme 14). ${ }^{36}$

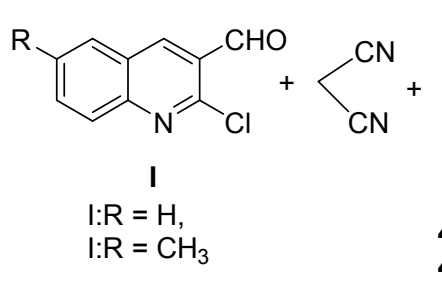

41a: $X=\mathrm{OH}, Y=\mathrm{H}$ 41b: $X=\mathrm{H}, \mathrm{Y}=\mathrm{OH}$

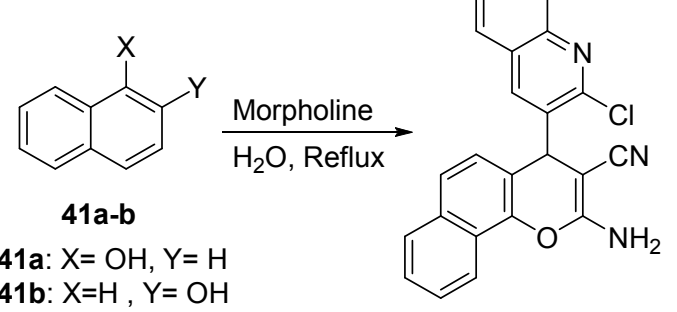

42a-b 42a: $\frac{\mathrm{R} \text { Yield(\%) }}{\mathrm{H} \quad 88}$ 42b $\quad \mathrm{CH}_{3} \quad 85$<smiles>[R]c1ccc2nc(Cl)c(C3C(C#N)=C(N)Oc4ccc5ccccc5c43)cc2c1</smiles>

42c-d 42c: $\begin{array}{ll}\frac{\mathrm{R}}{\mathrm{H}} & \text { Yield(\%) } \\ \text { 42d } & \mathrm{CH}_{3} 80\end{array}$

Scheme 14

Synthesis of highly substituted cyclopentadienes containing quinoline nucleus 46a-j, was described. The initially prepared Knöevenagel adducts 43a-b of 2-chloroquinoline-3-carbaldehydes and malononitrile or ethyl cyanoacetate underwent reaction with acetylenecarboxylates $44 a-b$ and isocyanide $45 a-b$ in dichloromethane at room temperature within $12 \mathrm{~h}$, affording the products in moderate to good yields. Mild reaction condition and prompt isolation of the products are some advantages of this protocol (Scheme 15). ${ }^{37}$ 


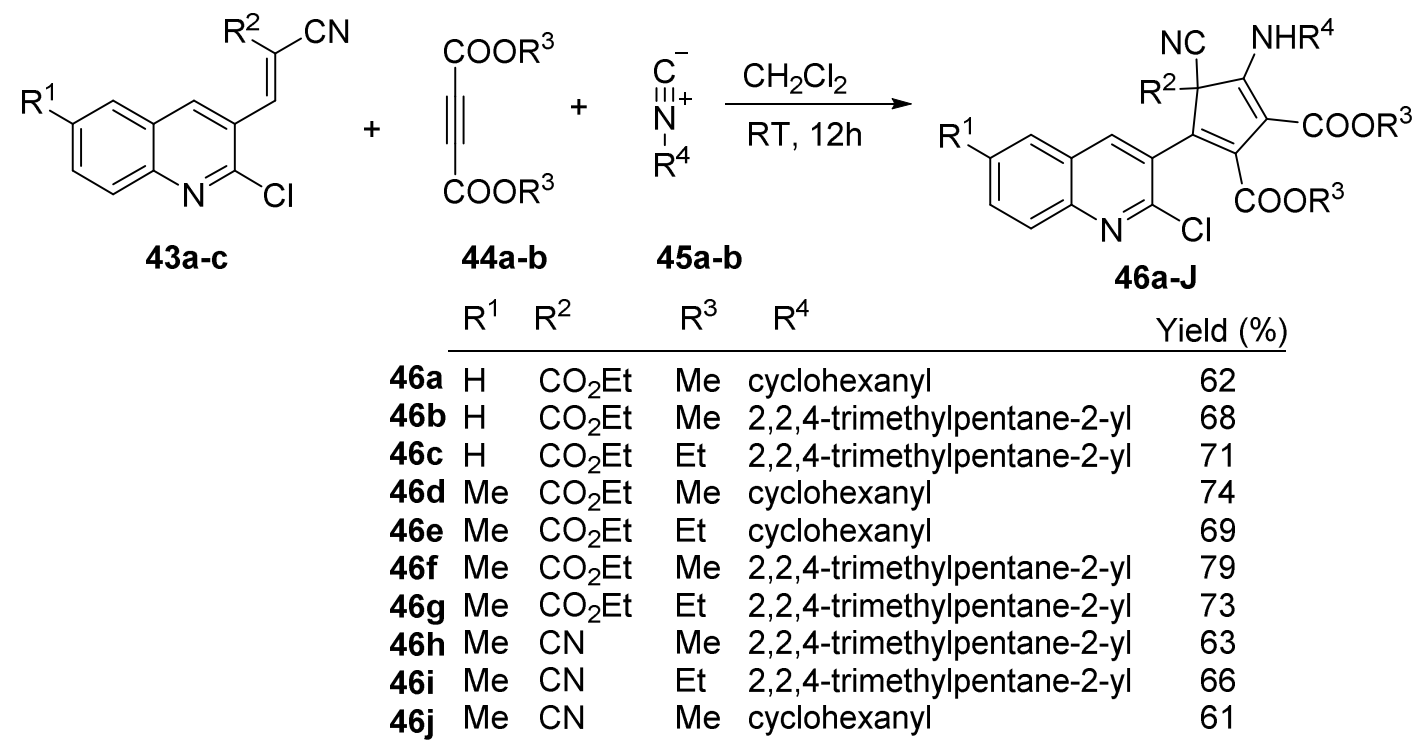

\section{Scheme 15}

When the aldehyde I was reacted with urea or thiourea $47 \mathbf{a}, \mathbf{b}$ and active methylene compounds $48 a-b$ in ethanol and in the presence of drops of acetic acid as a catalyst in one-pot reaction namely Biginelli reaction $^{38,39}$, the corresponding compounds $49 \mathbf{a}-\mathbf{b}$ and $54 \mathbf{a}-\mathbf{b}$ was obtained respectively. The carbohydrazide $\mathbf{5 0}$ was afforded by reaction of hydrazine hydrate with the ester derivative $\mathbf{4 9 b}$. Moreover, condensation of 49a with 2-aminophenol $\mathbf{5 1}$ in the presence of acetic acid afforded the tetrahydropyrimidine-5-carboxamide derivative 52; while the carbimidate derivative $\mathbf{5 3}$ was afforded, if the same reaction was carried out in ethanol (Scheme 16). ${ }^{40}$<smiles>[X]C(N)=[N+]=C(Cl)c1cc2ccccc2nc1C=O</smiles>

47<smiles>[X]C1=NC(c2cc3ccccc3nc2Cl)C(C(C)=O)=C(C)N1</smiles>

54a: $0,65 \%$

54b: S, $61 \%$<smiles>[R]C(=O)CC(C)=O</smiles>

48a-b 48a if $\mathbf{R}=\mathrm{OEt}$

(1)<smiles>CCC</smiles><smiles>[134IH]</smiles><smiles>CC1=C(C(=O)Nc2ccccc2O)C(c2cc3ccccc3nc2Cl)NC(=O)N1</smiles>

52, $73 \%$<smiles>[X]C1=NC(c2cc3ccccc3nc2Cl)C(C(=O)OCC)=C(C)N1</smiles><smiles>CCOC(=Nc1ccccc1O)C1=C(C)NC(=O)NC1c1cc2ccccc2nc1Cl</smiles>

53, $48 \%$ 
A series of 2-chloroquinoline-based imidazopyridines $\mathbf{5 7 a - i}$ and imidazothiazoles $\mathbf{5 7}$-o bearing a bulky alkylamine side chain were synthesized as soybean 15-LOX inhibitors. The target compounds of quinolinebased imidazole-fused heterocycles 57a-0 were prepared via one-pot reaction of 2-chloroquinoline-3carbaldehyde I, heteroaromatic amine $\mathbf{5 5}$, and alkyl isocyanides 56, in the presence of $\mathrm{NH}_{4} \mathrm{Cl}$ (Scheme 17). ${ }^{41}$

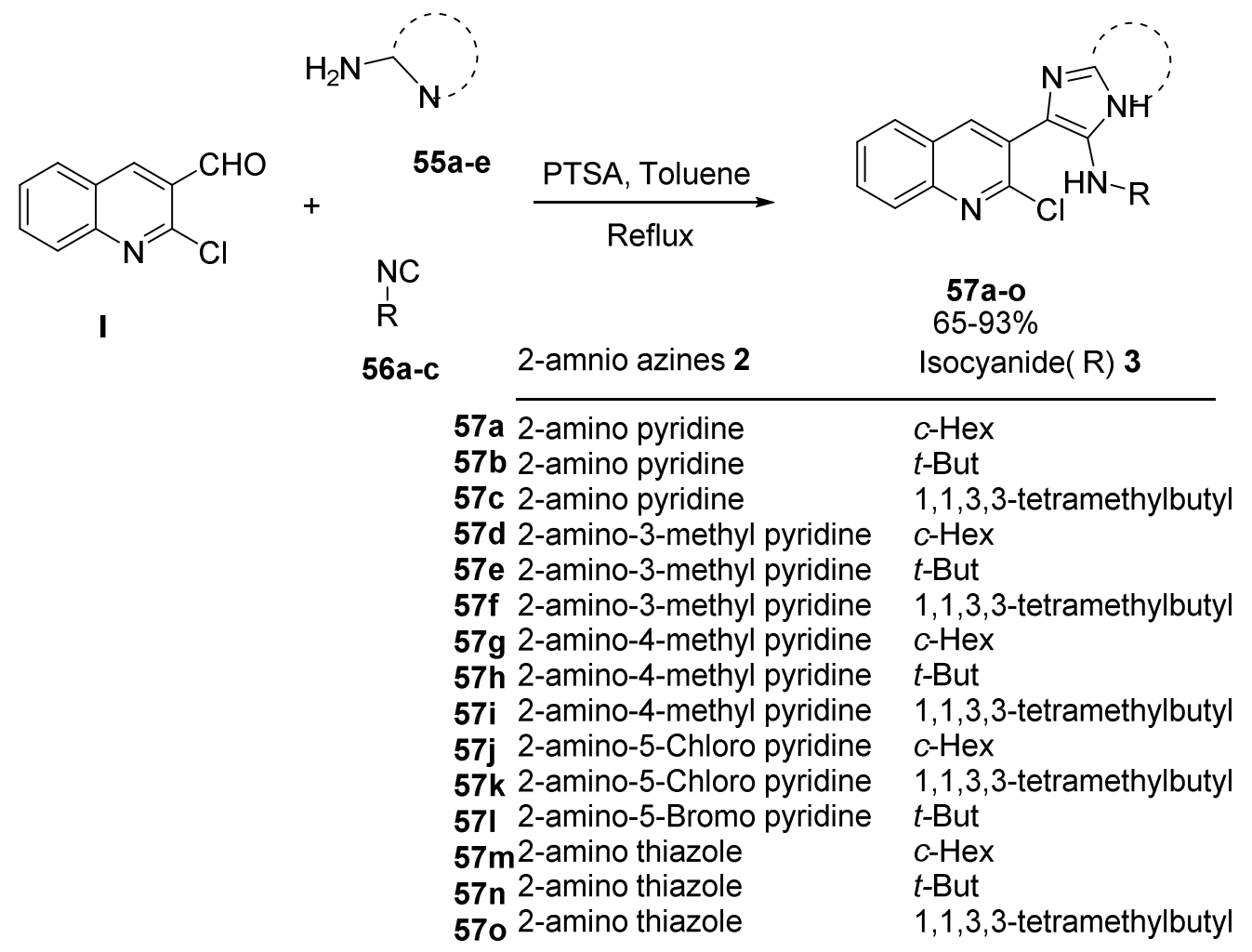

\section{Scheme 17}

Via Sonogashira conjoined electrophilic cyclization, the three component reaction of $o$-halo aldehydes I, alkynes 58a-e and tert-butylamine $\mathbf{5 9}$ led to the synthesis of biologically active benzo[b][1,6]naphthyridine derivatives 60a-h, using a bimetallic Pd/Cu catalytic system. (Scheme 18). ${ }^{42}$

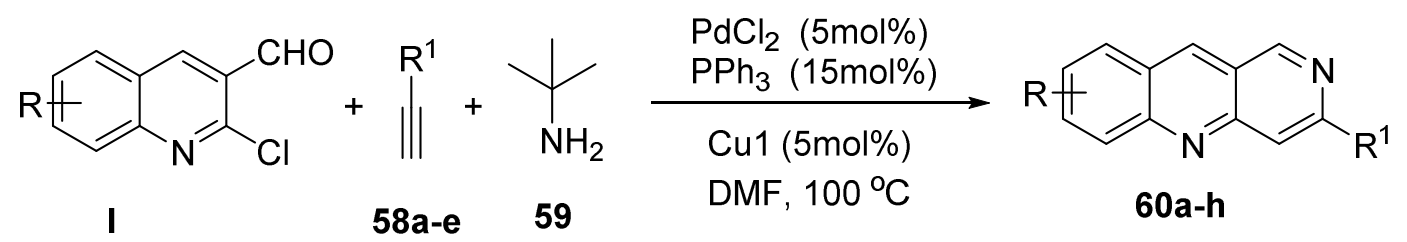

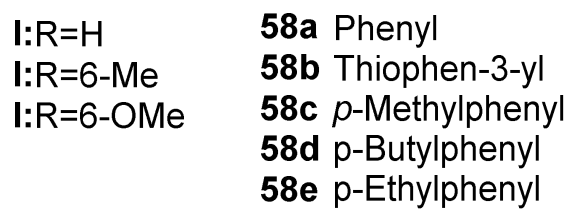

Scheme 18

\begin{tabular}{llc}
\multicolumn{1}{c}{$\mathrm{R}$} & $\mathrm{R}^{1}$ & Yield (\%) \\
\hline 60a H & Phenyl & 75 \\
60b H & $p$-Methylphenyl & 82 \\
60c H & $p$-Butylphenyl & 76 \\
60d H & Thiophen-3-yl & 80 \\
60e 6-Me & $p$-Methylphenyl & 78 \\
60f 6-Me & $p$-Ethylphenyl & 77 \\
60g 6-OMe & Phenyl & 77 \\
60h 6-OMe & p-Butylphenyl & 78
\end{tabular}


A facile and efficient method for synthesis of novel furylquinolines 62a-r was developed via the condensation of 2-chloroquinoline-3-carbaldehydes I with acetylenecarboxylates $\mathbf{4 4 a - b}$ and isocyanides $\mathbf{5 6} \mathbf{a}-\mathbf{b}$ and 61 . The mixture was stirred in acetonitrile for $12 \mathrm{~h}$ at $40{ }^{\circ} \mathrm{C}$. After completion of the reaction, the mixture was cooled to room temperature to afford 62a-r (Scheme 19). ${ }^{43}$

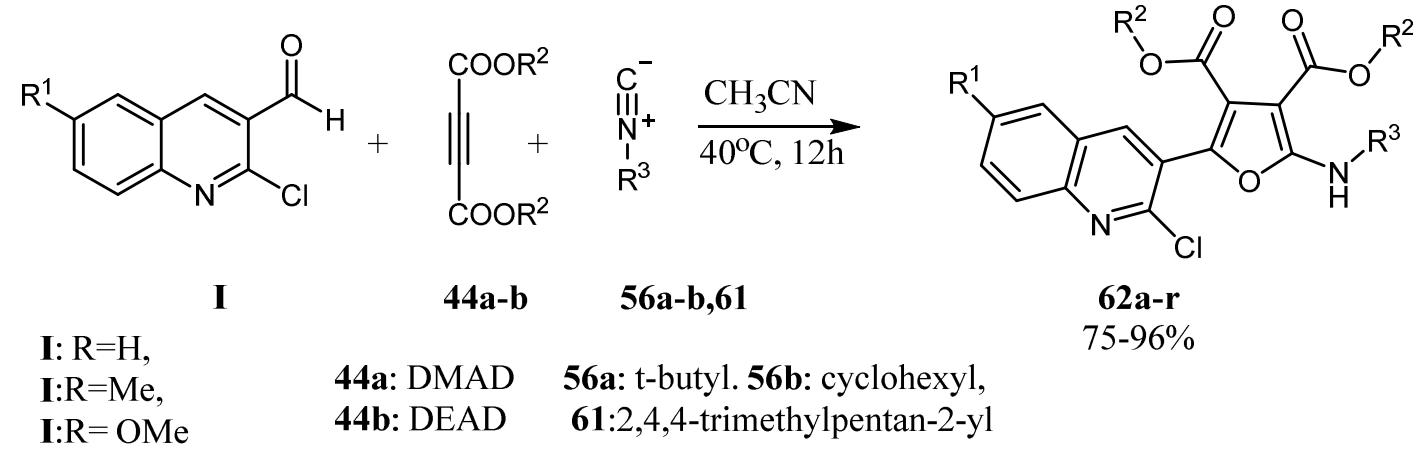

\section{Scheme 19}

3.1.3.2. Four component reactions. A convenient and facile method for synthesis of diverse quino[2,3b][1,5]benzoxazepines 66a-t was developed. The reaction proceeds through a one-pot sequential Ugi$4 \mathrm{CR} /$ base-free intramolecular aromatic nucleophilic substitution reaction $\left(S_{N} A r\right)$ in moderate to good yields from readily available starting materials. Upon treating 2-chloroquinoline-3-carbaldehyde $\mathbf{I}$ with 2aminophenol 63a, acetic acid 64a, and cyclohexyl isocyanide, 65a was directly obtained as a sole product in $83 \%$ yield (Scheme 20$){ }^{44}$

Also benzo[b][1,8]naphthyridine derivative $36\{7,1,5\}$ were obtained in low yield (42\% yield) via fourcomponent reaction of 6-tert-butyl-2-chloroquinoline-3-carbaldehyde 1(7), 4-hydroxycoumarin 34(1), dimedone, and aniline. After completion of the reaction, the reaction mixture was cooled to room temperature. The crystalline solids were collected and purified by recrystallization from DMF and water to give pure products $\mathbf{3 6}\{\mathbf{7 , 1 , 5 \}}$ (Scheme 21$) .^{34}$

In an extended work at the same context, the synthesis of diverse naphthyridinone derivatives 68a-h was presented. When 2-chloroquinoline-3-carbaldehydes I, 3-methyl-1H-pyrazol-5(4H)-one 67, enaminone 35, $L$-proline was stirred and refluxed in ethanol (Scheme 22). ${ }^{45}$ 
<smiles>[R]c1ccc2nc(Cl)c(C=O)cc2c1</smiles>

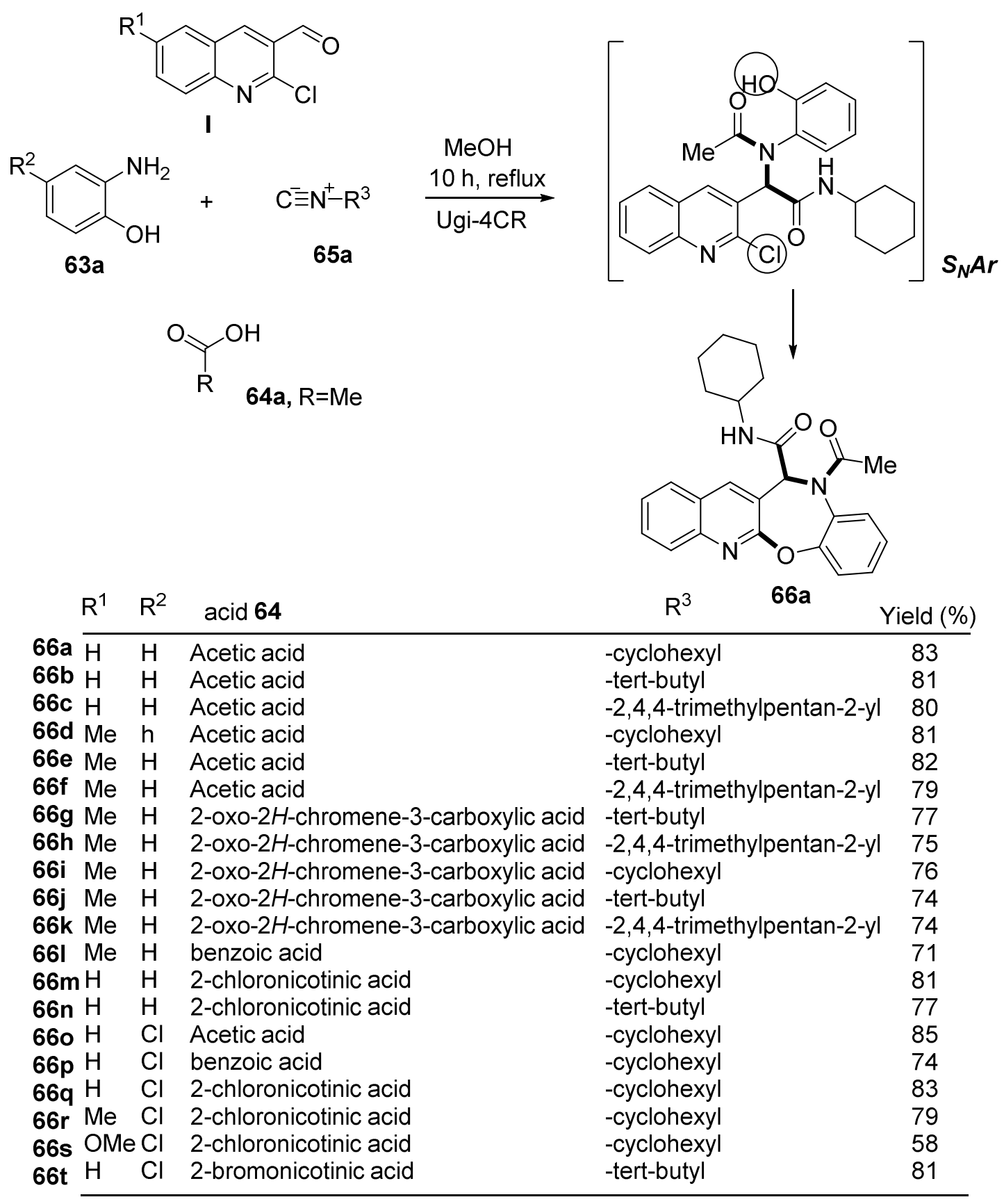

\section{Scheme 20}

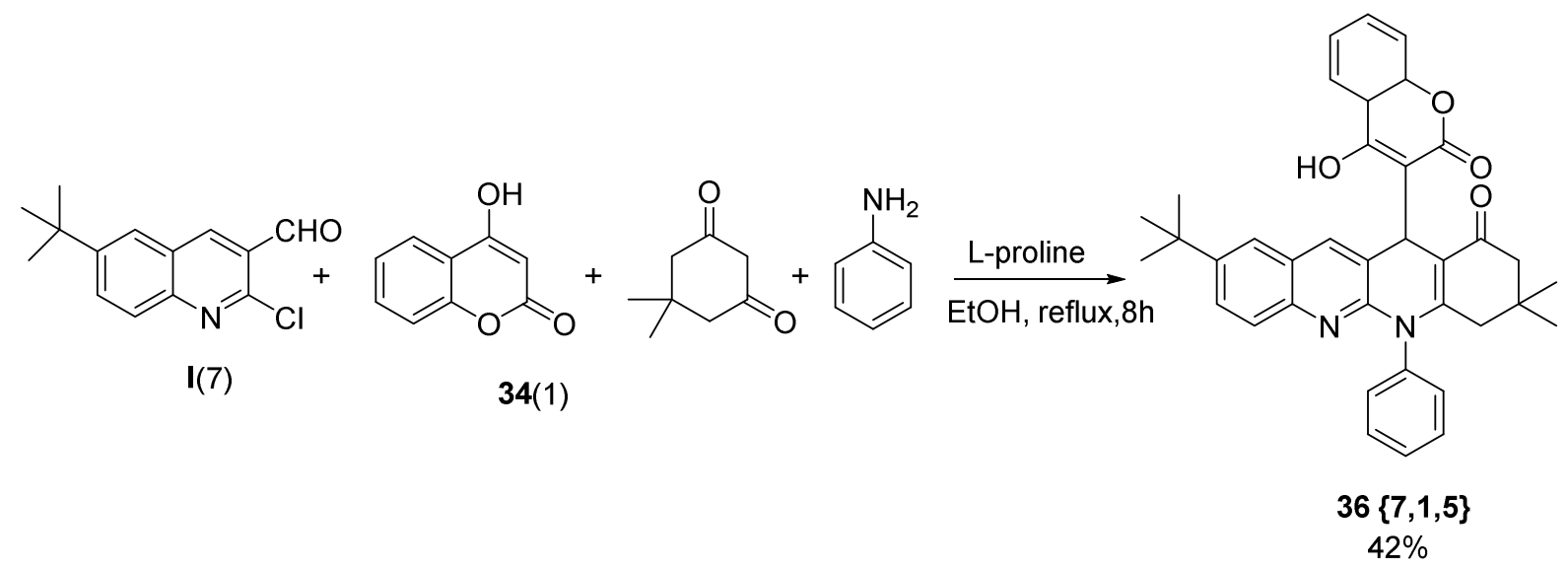




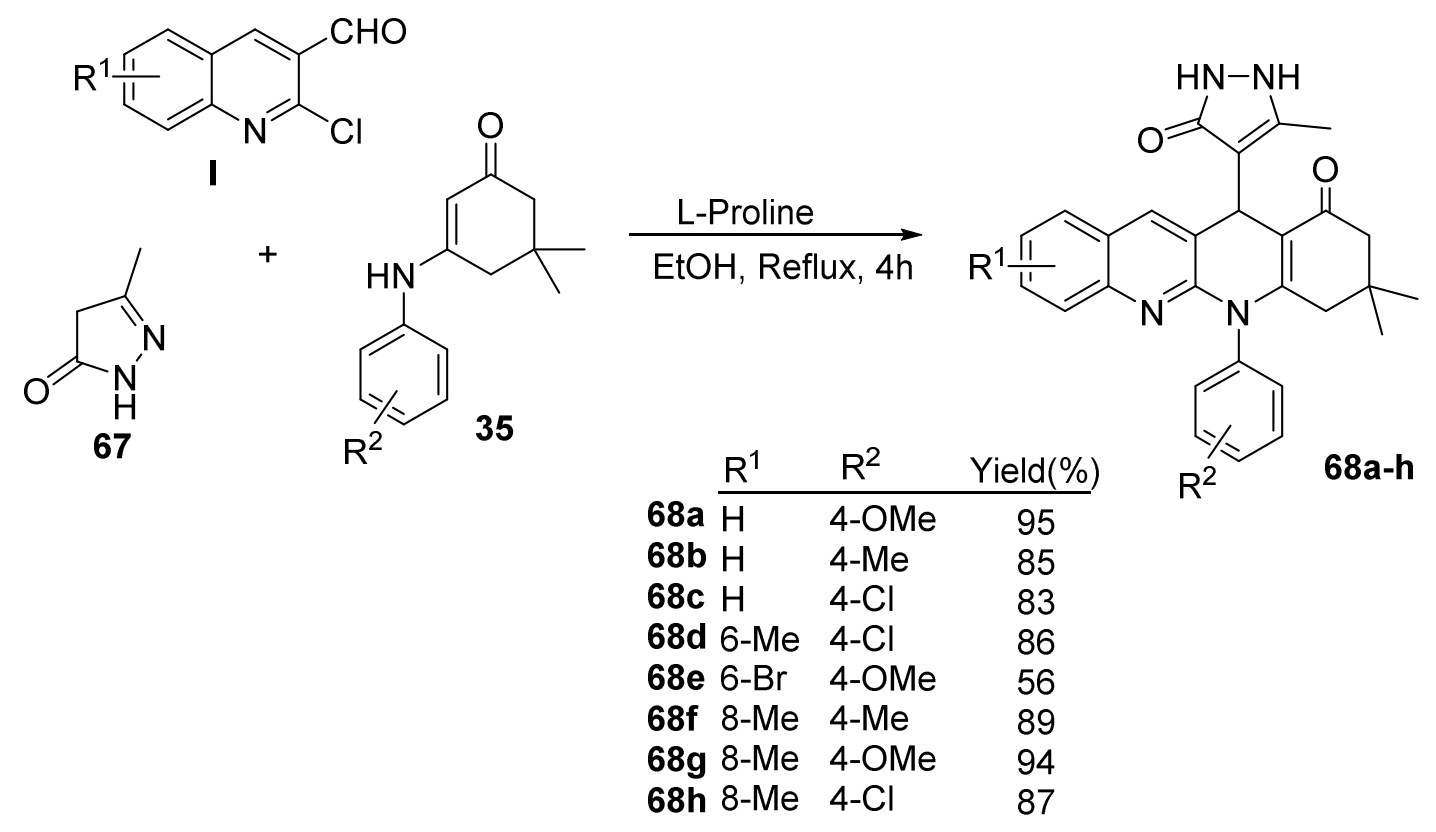

\section{Scheme 22}

A one-pot reaction providing quinoline-based 1,4-dihydropyridines 71a-t was developed through reaction of 2-chloroquinoline-3-carbaldehyde I and malononitrile or ethyl cyanoacetate 69a-b in EtOH, TEA followed by addition of amine 70a-f and dialkylacetylenedicarboxylate 44a-b (Scheme 23 ). ${ }^{46}$

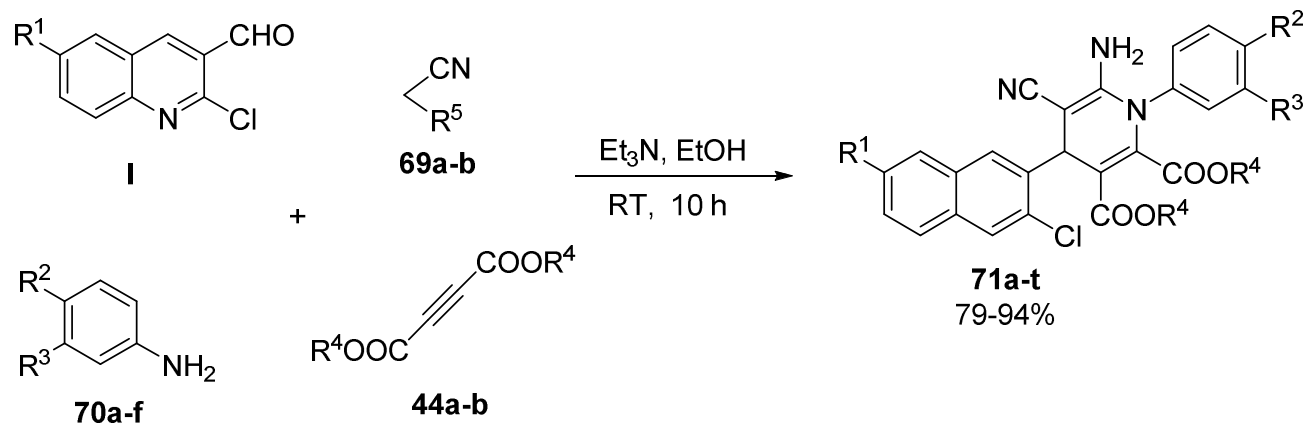

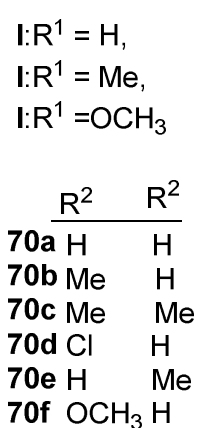

\begin{tabular}{llllll} 
& $\mathrm{R}^{1}$ & $\mathrm{R}^{2}$ & $\mathrm{R}^{3}$ & $\mathrm{R}^{4}$ & $\mathrm{R}^{5}$ \\
\cline { 2 - 5 } 71a & $\mathrm{H}$ & $\mathrm{H}$ & $\mathrm{H}$ & $\mathrm{Me}$ & $\mathrm{CN}$ \\
71b & $\mathrm{H}$ & $\mathrm{Me}$ & $\mathrm{H}$ & $\mathrm{Me}$ & $\mathrm{CN}$ \\
71c & $\mathrm{H}$ & $\mathrm{Me}$ & $\mathrm{Me}$ & $\mathrm{Me}$ & $\mathrm{CN}$ \\
71d & $\mathrm{Me}$ & $\mathrm{Cl}$ & $\mathrm{H}$ & $\mathrm{Me}$ & $\mathrm{CN}$ \\
71e & $\mathrm{Me}$ & $\mathrm{Me}$ & $\mathrm{Me}$ & $\mathrm{Me}$ & $\mathrm{CN}$ \\
71f & $\mathrm{OCH}_{3}$ & $\mathrm{H}$ & $\mathrm{H}$ & $\mathrm{Me}$ & $\mathrm{CN}$ \\
71g & $\mathrm{OCH}_{3}$ & $\mathrm{H}$ & $\mathrm{Me}$ & $\mathrm{Me}$ & $\mathrm{CN}$ \\
71h & $\mathrm{OCH}_{3}$ & $\mathrm{Cl}$ & $\mathrm{H}$ & $\mathrm{Me}$ & $\mathrm{CN}$ \\
71i & $\mathrm{H}$ & $\mathrm{OCH}$ & $\mathrm{H}$ & $\mathrm{Et}$ & $\mathrm{CN}$ \\
71j & $\mathrm{H}$ & $\mathrm{Me}$ & $\mathrm{H}$ & $\mathrm{Et}$ & $\mathrm{CN}$
\end{tabular}

\begin{tabular}{|c|c|c|c|c|}
\hline $\mathrm{R}^{1}$ & $\mathrm{R}^{2}$ & $\mathrm{R}^{3}$ & $\mathrm{R}^{4}$ & $\mathrm{R}^{5}$ \\
\hline 71k H & $\mathrm{H}$ & $\mathrm{H}$ & Me & $-\mathrm{CO}_{2} \mathrm{Et}$ \\
\hline $71 \mathrm{H}$ & $\mathrm{Me}$ & $\mathrm{H}$ & $\mathrm{Me}$ & $-\mathrm{CO}_{2} \mathrm{Et}$ \\
\hline $71 \mathrm{~m} \mathrm{H}$ & Me & $\mathrm{Me}$ & Me & $\mathrm{O}_{2} \mathrm{Et}$ \\
\hline 71n Me & $\mathrm{Cl}$ & $\mathrm{H}$ & $\mathrm{Me}$ & $-\mathrm{CO}_{2} \mathrm{Et}$ \\
\hline $710 \mathrm{Me}$ & $\mathrm{Me}$ & $\mathrm{Me}$ & $\mathrm{Me}$ & \\
\hline $71 p \mathrm{OCH}_{3}$ & $\mathrm{H}$ & $\mathrm{H}$ & $\mathrm{Me}$ & $\mathrm{O}_{2} \mathrm{Et}$ \\
\hline $71 q \mathrm{OCH}_{3}$ & $\mathrm{H}$ & $\mathrm{Me}$ & $\mathrm{Me}$ & $-\mathrm{CO}_{2} \mathrm{Et}$ \\
\hline $71 \mathrm{r} \mathrm{OCH}_{3}$ & $\mathrm{Cl}$ & $\mathrm{H}$ & $\mathrm{Me}$ & $-\mathrm{CO}_{2} \mathrm{Et}$ \\
\hline 71s $\mathrm{H}$ & $\mathrm{OCH}_{3}$ & $\mathrm{H}$ & Et & $-\mathrm{CO}_{2} \mathrm{Et}$ \\
\hline 71t $\mathrm{H}$ & Me & $\mathrm{H}$ & Et & \\
\hline
\end{tabular}

\section{Scheme 23}

Recently, an one-pot method has been used for the synthesis of new polycyclic compounds articulated around 3-cyanopyridine derivatives 73a-f and 74a-d from 2-chloroquinolin-3- carbaldehydes I, acetophenone derivatives 72, active methylene compounds 69a-b, and ammonium acetate as a source of ammonia in the presence of catalytic amounts of $\mathrm{PPh}_{3}$ at room temperature (Scheme 24). ${ }^{47}$ 
<smiles>[R]c1c([R])c([R])c2nc(Cl)c(C=O)cc2c1[R]</smiles><smiles>CCOC(=O)OCCC(C)[Y]=N</smiles><smiles>[R]c1c([R])c([R])c2nc(Cl)c(-c3cc(-c4cc[R]([H])cc4)nc(N)c3C)cc2c1[R]</smiles><smiles>[R]c1ccc(-c2cc(-c3cc4c([R])c([R])c([R])c([R])c4nc3Cl)c(C#N)c(=N)[nH]2)cc1</smiles><smiles>[R]c1c([R])c([R])c2nc(Cl)c(-c3cc(-c4cc[R1]([H])cc4)[nH]c(=O)c3C#N)cc2c1[R]</smiles>

\begin{tabular}{|c|c|c|c|}
\hline $\mathrm{R}^{1}$ & $\mathrm{R}^{2}$ & $\mathrm{R}^{3}$ & $\mathrm{R}^{4}$ \\
\hline I: H & $\mathrm{H}$ & $\mathrm{H}$ & $\mathrm{H}$ \\
\hline I: H & OMe & $\mathrm{H}$ & $\mathrm{H}$ \\
\hline I: H & $\mathrm{H}$ & OMe & $\mathrm{H}$ \\
\hline I: OMe & $\mathrm{H}$ & $\mathrm{H}$ & $\mathrm{OMe}$ \\
\hline I: H & $\mathrm{H}$ & $\mathrm{Me}$ & $\mathrm{H}$ \\
\hline
\end{tabular}

\begin{tabular}{llllllc} 
& $\mathrm{R}^{1}$ & $\mathrm{R}^{2}$ & $\mathrm{R}^{3}$ & $\mathrm{R}^{4}$ & $\mathrm{R}$ & Yield (\%) \\
\cline { 2 - 6 } 73a & $\mathrm{H}$ & $\mathrm{H}$ & $\mathrm{H}$ & $\mathrm{H}$ & $\mathrm{H}$ & 28 \\
73b & $\mathrm{H}$ & $\mathrm{OMe}$ & $\mathrm{H}$ & $\mathrm{H}$ & $\mathrm{H}$ & 40 \\
73c & $\mathrm{H}$ & $\mathrm{H}$ & $\mathrm{Me}$ & $\mathrm{H}$ & $\mathrm{H}$ & 43 \\
73d & $\mathrm{OMe}$ & $\mathrm{H}$ & $\mathrm{H}$ & $\mathrm{OMe}$ & $\mathrm{OMe}$ & 38 \\
73e & $\mathrm{H}$ & $\mathrm{H}$ & $\mathrm{H}$ & $\mathrm{H}$ & $\mathrm{OMe}$ & 36 \\
73f & $\mathrm{H}$ & $\mathrm{OMe}$ & $\mathrm{H}$ & $\mathrm{H}$ & OMe & 33 \\
74a & $\mathrm{H}$ & $\mathrm{H}$ & $\mathrm{Me}$ & $\mathrm{H}$ & $\mathrm{OMe}$ & 44 \\
74b & $\mathrm{H}$ & $\mathrm{OMe}$ & $\mathrm{H}$ & $\mathrm{H}$ & OMe & 36 \\
74c & $\mathrm{H}$ & $\mathrm{H}$ & $\mathrm{OMe}$ & $\mathrm{H}$ & OMe & 33 \\
74d & OMe & $\mathrm{H}$ & $\mathrm{H}$ & OMe & OMe & 31
\end{tabular}

\section{Scheme 24}

\subsection{Reduction of the aldehyde group}

A simple and high yielding method was developed for the synthesis of new (2-chloroquinolin-3-yl)methyl diethyl phosphate 76a-h from the corresponding alcohol derivatives $\mathbf{7 5 a} \mathbf{h}$, that obtained from the aldehydes $\mathrm{I}$, by using 0,0 -diethyl chlorophosphate in the presence of $\mathrm{NaOH}$ and methylene chloride at ambient temperature (Scheme 25). ${ }^{48}$ 
<smiles>[R]c1cc2cc(C=O)c(Cl)nc2c([R])c1[R]</smiles><smiles>[R]c1cc2cc(CO)c(Cl)nc2c([R])c1[R]</smiles>

\begin{tabular}{|c|c|c|c|c|}
\hline & $\mathrm{R}^{1}$ & $\mathrm{R}^{2}$ & $\mathrm{R}^{3}$ & Yield 76(\%) \\
\hline $76 a$ & $\mathrm{H}$ & $\mathrm{H}$ & $\mathrm{H}$ & 92 \\
\hline $76 b$ & $\mathrm{CH}_{3}$ & $\mathrm{H}$ & $\mathrm{H}$ & 93 \\
\hline $76 c$ & & $\mathrm{CH}_{3}$ & $\mathrm{H}$ & 93 \\
\hline $76 d$ & & $\mathrm{H}$ & $\mathrm{CH}_{3}$ & 91 \\
\hline $76 e$ & $\mathrm{OCH}_{3}$ & $\mathrm{H}$ & $\mathrm{H}^{\circ}$ & 90 \\
\hline & & $\mathrm{OCH}_{3}$ & $\mathrm{H}$ & 92 \\
\hline & $\mathrm{OC}_{2} \mathrm{H}_{5}$ & $\mathrm{H}$ & $\mathrm{H}$ & 94 \\
\hline & & $\mathrm{H}$ & $\mathrm{OC}_{2} \mathrm{H}_{5}$ & 591 \\
\hline
\end{tabular}<smiles>[R]c1cc2cc(COP(=O)(OCC)OCC)c(Cl)nc2c([R])c1[R]</smiles>

\section{Scheme 25}

The synthesis of a new series of 2-chloroquinolin-3-yl ester derivatives 77a-i was reported via a twosteps protocol from 2-chloroquinoline-3-carbaldehydes I. Firstly, I was reduced using $\mathrm{NaBH}_{4}$ in methanol to yield the corresponding alcohol derivatives $\mathbf{7 5 a}, \mathbf{b}, \mathbf{e}$, which is then reacted with acid chloride in DMF along with activated $\mathrm{K}_{2} \mathrm{CO}_{3}$ at room temperature to afford target compounds (Scheme 26). ${ }^{49}$

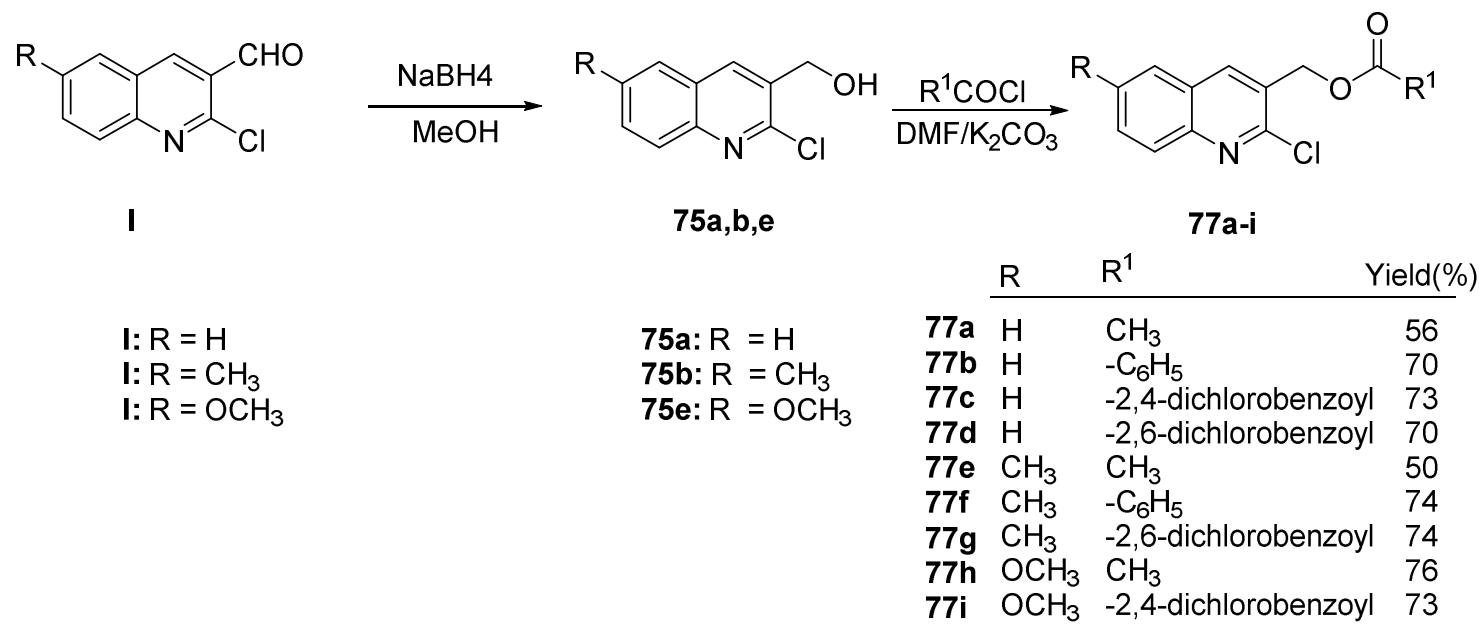

\section{Scheme 26}

Condensation of 2-chloroquinoline-3-carbaldehyde I with $\mathrm{NaN}_{3}$ in DMF afforded tetrazolo[1,5a]quinoline-4-carbaldehyde 78, which was reduced by $\mathrm{NaBH}_{4}$ to obtain tetrazolo[1,5-a]quinolin-4-yl methanol 79 (Scheme 27). ${ }^{50,51}$

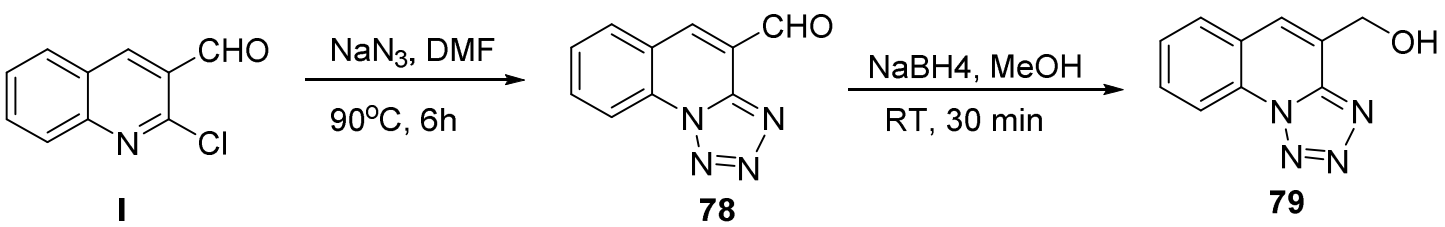

Scheme 27 
The quinolinyl methanol 79, when allowed to react with methane sulfonyl chloride in presence of TEA in $\mathrm{DCM}$ at $0{ }^{\circ} \mathrm{C}$, yielded the corresponding tetrazolo[1,5-a]quinolin-4-ylmethyl methanesulfonate ester 80, which on condensation with p-hydroxybenzaldehyde in DMF in presence of $\mathrm{K}_{2} \mathrm{CO}_{3}$, afforded the required precursor aldehyde $\mathbf{8 1}$. One-pot cyclocondensation of the $\mathbf{8 1}$ with anilines $\mathbf{8 2 a - I}$ and mercaptoacetic acid $\mathbf{8 3}$ was carried out in PEG-400 at $110{ }^{\circ} \mathrm{C}$ to obtain the thiazolidin-4-ones $84 a-I$ in moderate to good yield (Scheme 28). ${ }^{52}$

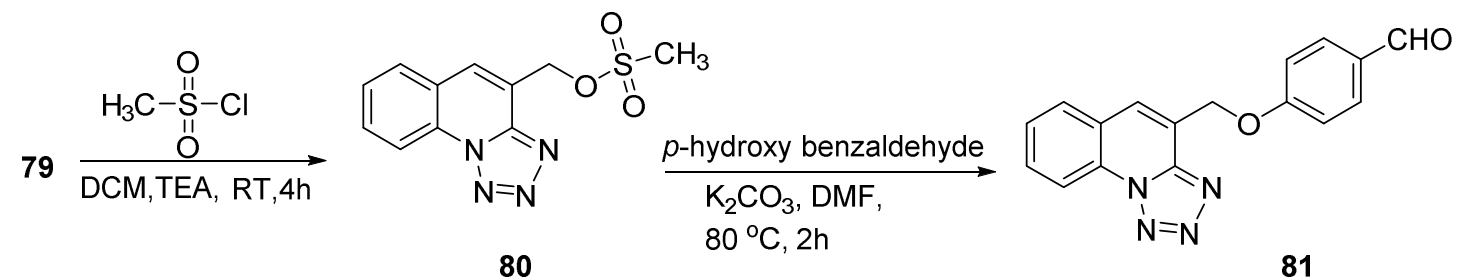

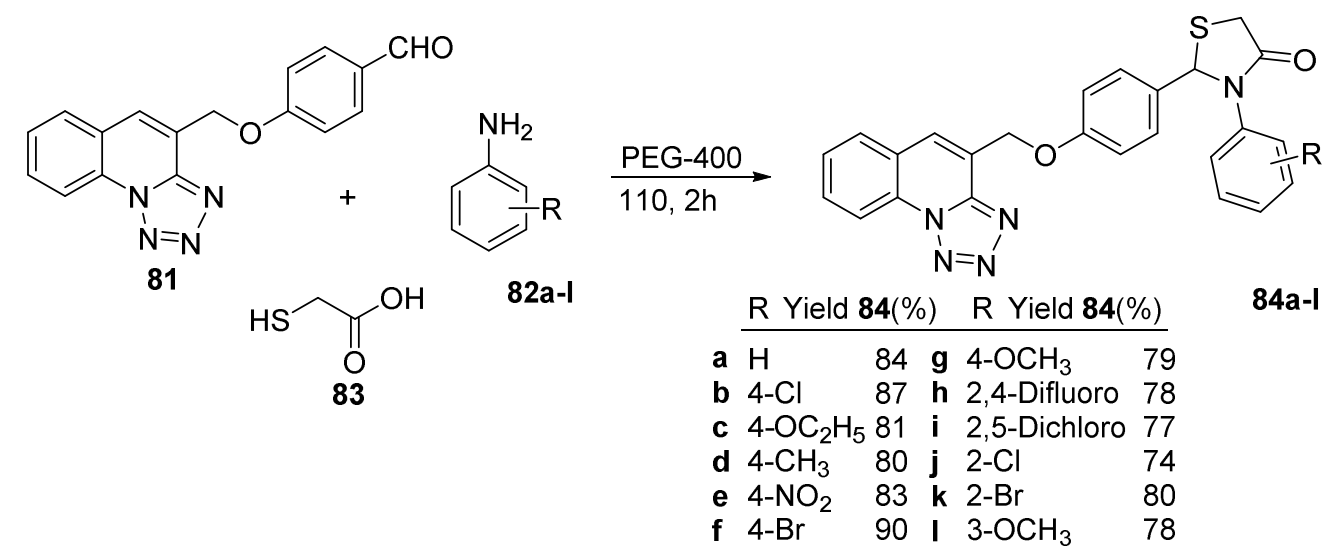

\section{Scheme 28}

\subsection{Oxidation of the aldehyde group}

2-Chloroquinoline-3-carboxylic acid was prepared by oxidation of I using silver nitrate in the presence of sodium hydroxide. ${ }^{53}$ Esterification of the carboxylic acid derivative $\mathbf{8 5}$ using absolute ethanol and sulfuric acid afforded the ester derivative $\mathbf{8 6}$, in a good yield, followed by subsequent hydrazinolysis in boiling ethanol to afford 2-chloroquinoline-3-carbohydrazide 87. The later compound $\mathbf{8 7}$ was subjected to react with carbon disulfide in ethanol in the presence of $\mathrm{KOH}$ under reflux followed by acidification using diluted $\mathrm{HCl}$ to give 5-(2chloro-quinolin-3-yl)-1,3,4- oxadiazole-2-thiol 88 (Scheme 29). ${ }^{6}$
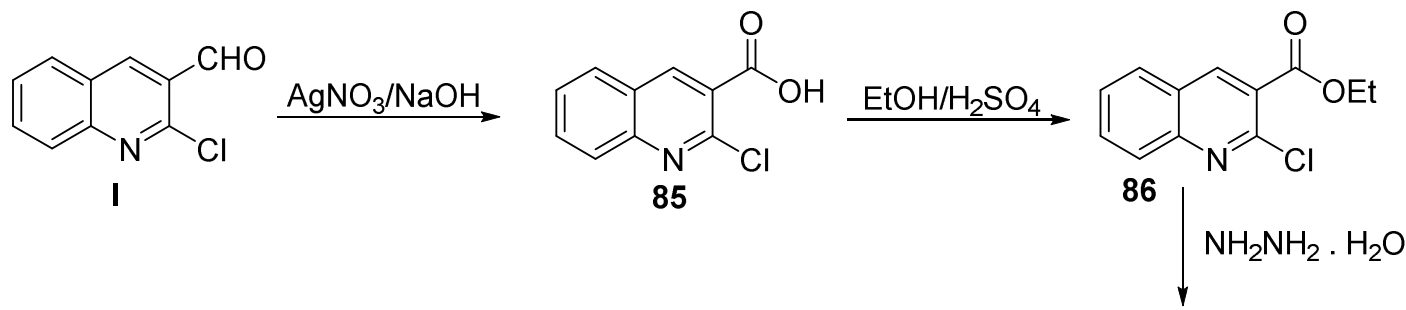<smiles>Sc1nnc(-c2cc3ccccc3nc2Cl)o1</smiles>

$88,78 \%$

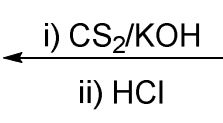

ii) $\mathrm{HC}$

Scheme 29 


\subsection{Condensation reactions}

3.4.1. Reactions with active methylene compounds. A series of $\alpha, \beta$-unsaturated carbonyl compounds 89a-b and $\mathbf{9 0}$ were synthesized through the Claisen-Schmidt condensation of equimolar amounts of the aldehydes $\mathbf{I}$ with different active methylene compounds and methyl respectively, in ethanol in the presence of $\mathrm{NaOH}$ as catalyst. In addition, the Knoevenagel condensation of I with 2-methyl-4H-benzo[d][1,3]oxazin-4-one led to the formation of non-isolable quionoline intermediate 91', which underwent cyclization via elimination of $\mathrm{HCl}$ to afford 2-(2- oxobenzo[b][1,8]naphthyridin-1(2H)-yl)benzoic acid $\mathbf{9 1}$. While the azlactone 92 was obtained by reaction of $\mathrm{N}$-acetylglycine with $\mathrm{I}$ in the presence of acetic anhydride and sodium acetate (Scheme 30). ${ }^{54}$

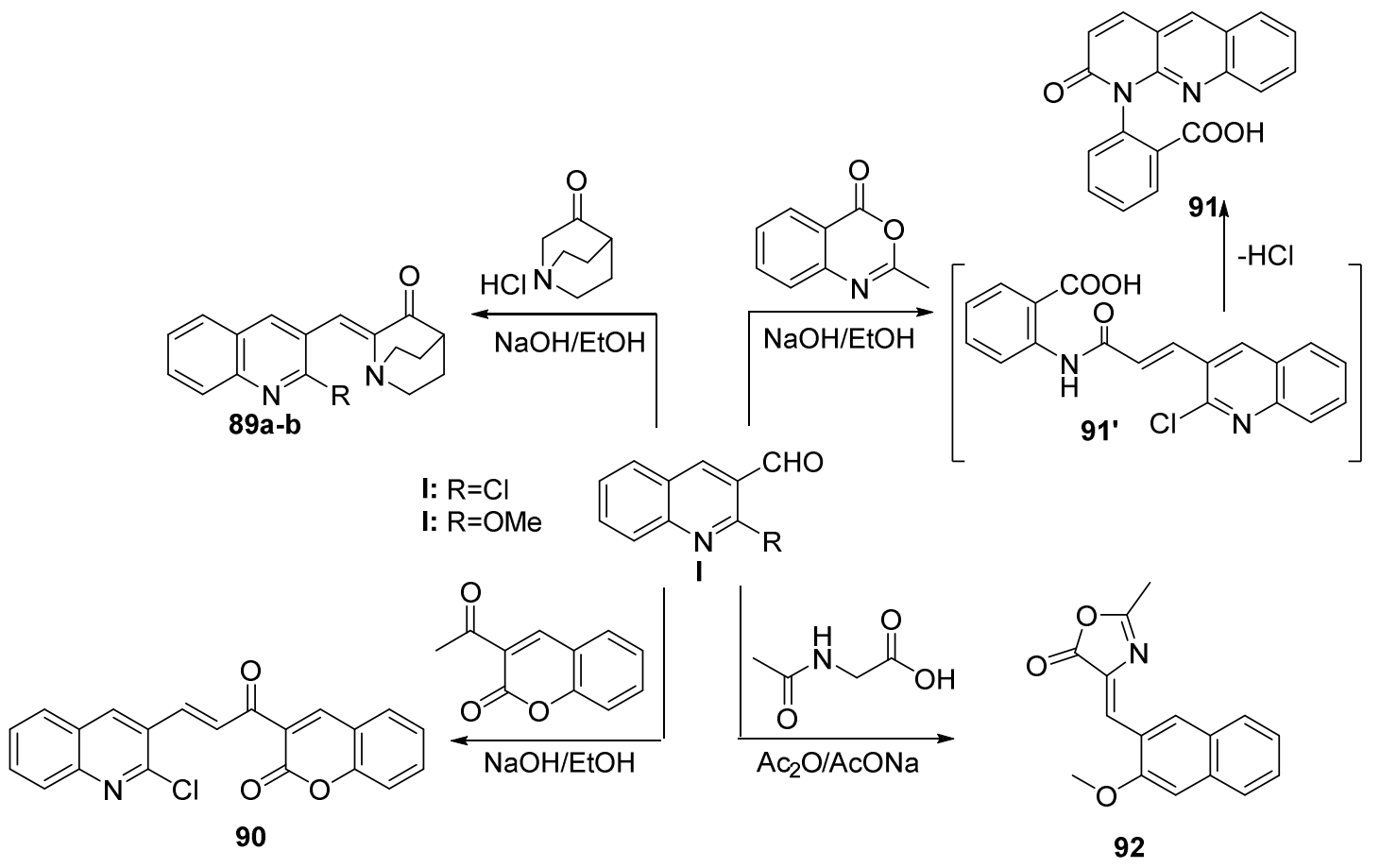

\section{Scheme 30}

Substituted 2-chloroquinoline-3-carbaldehydes I, or substituted 2-(piperidin-1-ylmorpholino) quinoline3-carbaldehydes $\mathbf{9 3 ( 1 - 5 )}$ were reacted with rhodanine derivatives $\mathbf{9 4 ( 1 - 3 )}$ in the presence of sodium acetate and acetic acid to afford new rhodanine analogs 95(1-31) as anticancer agents. Replacement of chlorine group with piperazine or morpholine, improved anticancer activity. While, replacement of rhodanine with rhodanine acetic acid does not change much in the anticancer activity (Scheme 31). ${ }^{55}$ 
<smiles>[R]c1nc2c([R])c([R])c([R])c([R7])c2cc1C=O</smiles><smiles>CC(=O)CCCCOC(C)=O</smiles>

\begin{tabular}{lll} 
& \multicolumn{2}{c}{$\mathrm{R}^{1}$} \\
\cline { 2 - 3 } $94(1)$ & $\mathrm{H}$ \\
$94(2)$ & $-\mathrm{CH}_{2} \mathrm{COOH}$ \\
$94(3)$ & $-\mathrm{ph}$ & \\
& \multicolumn{1}{c}{$\mathrm{I}$} & 94 \\
\cline { 2 - 3 } $95(1)$ & $\mathrm{I}(1)$ & $94(1)$ \\
$95(2)$ & $\mathrm{I}\left(2^{\prime}\right)$ & $94(1)$ \\
$95(3)$ & $\mathrm{I}\left(3^{\prime}\right)$ & $94(1)$ \\
$95(4)$ & $\mathrm{I}\left(4^{\prime}\right)$ & $94(1)$ \\
$95(5)$ & $\mathrm{I}\left(5^{\prime}\right)$ & $94(1)$ \\
$95(6)$ & $\mathrm{I}\left(6^{\prime}\right)$ & $94(1)$ \\
$95(7)$ & $\mathrm{I}\left(7^{\prime}\right)$ & $94(1)$ \\
$95(8)$ & $\mathrm{I}\left(8^{\prime}\right)$ & $94(1)$ \\
$95(9)$ & $\mathrm{I}\left(9^{\prime}\right)$ & $94(1)$ \\
$95(10)$ & $\mathrm{I}\left(10^{\prime}\right)$ & $94(1)$ \\
$95(11)$ & $\mathrm{I}\left(11^{\prime}\right)$ & $94(1)$ \\
$95(12) \mathrm{I}\left(12^{\prime}\right)$ & $94(1)$
\end{tabular}

95(11) l(11') 94(1)<smiles>[R]c1nc2c([R])c([R])c([R5])c([R7])c2cc1/C=C1\SC(=S)N([R1])C1=O</smiles>

95(1-31) $66-85 \%$

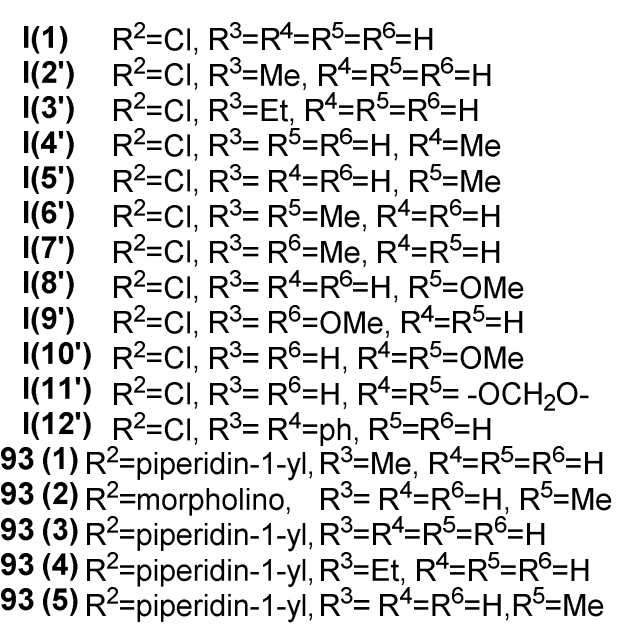

I(1) $R^{2}=\mathrm{Cl}, \mathrm{R}^{3}=\mathrm{R}^{4}=\mathrm{R}^{5}=\mathrm{R}^{6}=\mathrm{H}$

(12) $R^{2}=C l, R^{3}=M e, R^{4}=R^{5}=R^{6}=H$

$R^{2}=C R^{3}=R^{5}=R^{6}=H^{4} R^{4}=$

I(5) $\quad R^{2}=\mathrm{Cl}, \mathrm{R}^{3}=\mathrm{R}^{4}=\mathrm{R}^{6}=\mathrm{H}, \mathrm{R}^{5}=\mathrm{Me}$

I(6') $R^{2}=\mathrm{Cl}, \mathrm{R}^{3}=\mathrm{R}^{5}=\mathrm{Me}, \mathrm{R}^{4}=\mathrm{R}^{6}=\mathrm{H}$

$R^{2}=C l, R^{3}=R^{6}=M e, R^{4}=R^{5}=H$

I(10') $R^{2}=\mathrm{Cl}, \mathrm{R}^{3}=\mathrm{R}^{6}=\mathrm{H}, \mathrm{R}^{4}=\mathrm{R}^{5}=\mathrm{OM}$

I(11') $\mathrm{R}^{2}=\mathrm{Cl}, \mathrm{R}^{3}=\mathrm{R}^{6}=\mathrm{H}, \mathrm{R}^{4}=\mathrm{R}^{5}=-\mathrm{OCH}_{2} \mathrm{O}$

I(12') $R^{2}=\mathrm{Cl}, \mathrm{R}^{3}=\mathrm{R}^{4}=\mathrm{ph}, \mathrm{R}^{5}=\mathrm{R}^{6}=\mathrm{H}$

93 (3) $R^{2}=$ piperidin-1-yl, $R^{3}=R^{4}=R^{5}=R^{6}=H$

93 (5) $R^{2}=$ piperidin-1-yl, $R^{3}=R^{4}=R^{6}=H, R^{5}=M e$

\section{Scheme 31}

An efficient, eco-friendly method for rapid Knoevenagel condensation of 2-chloroquinoline-3carbaldehydes I with ethyl cynoacetate 69b under ultrasonic irradiation in solvent-free medium by using $N$ ethyl diisopropyl amine (NEDA) as catalyst within short time period (14-20 min) at room temperature was reported. Compared with traditional method, this method is more convenient and reaction can be carried out in higher yield, shorter reaction time and milder condition, without generation of pollution and safer to analyst (Scheme 32). ${ }^{56}$

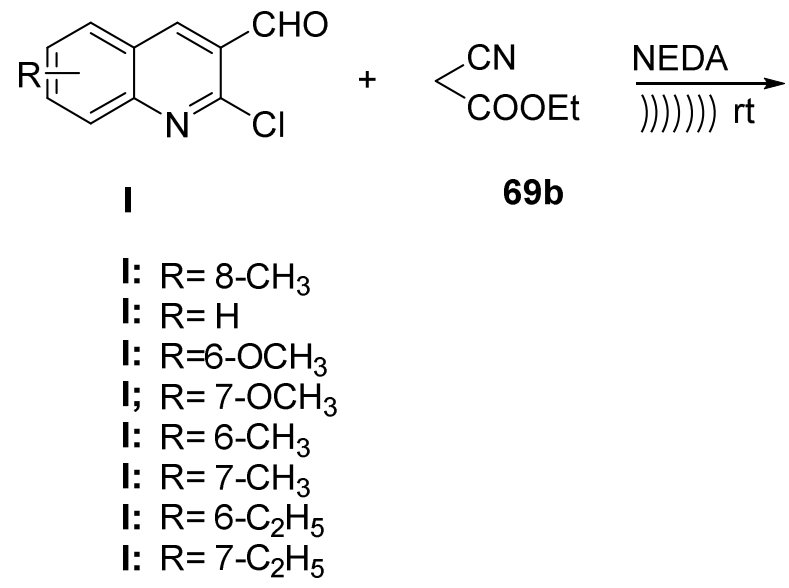<smiles></smiles>

\begin{tabular}{|c|c|c|}
\hline 96a-h & $\mathrm{R} \quad \mathrm{Y}$ & Yield (\%) \\
\hline $96 a$ & $8-\mathrm{CH}_{3}$ & 89 \\
\hline $96 b$ & $\mathrm{H}$ & 85 \\
\hline $96 c$ & $6-\mathrm{OCH}_{3}$ & 88 \\
\hline 96d & $7-\mathrm{OCH}_{3}$ & 84 \\
\hline $96 e$ & $6-\mathrm{CH}_{3}$ & 87 \\
\hline $96 f$ & $7-\mathrm{CH}_{3}$ & 89 \\
\hline $96 \mathrm{~g}$ & $6-\mathrm{C}_{2} \mathrm{H}_{5}$ & 82 \\
\hline $96 h$ & $1-2+15$ & 91 \\
\hline
\end{tabular}

\section{Scheme 32}

In addition, a facile, efficient and green methodology for the Knoevenagel condensation reaction was reported by grinding a mixture of hetero aryl aldehydes I and various active methylene compounds $69 \mathbf{a}, \mathbf{b}$ and $\mathbf{9 7 a , b}$ with catalytic amount of $[\mathrm{bnmim}] \mathrm{OH}$, at room temperature. The product was extracted twice from 
diethyl ether, leaving behind [bnmim]OH. Organic layer washed by brine solution $(2 \times 10 \mathrm{~mL})$ and dried over sodium sulfate and the solvent was evaporated under reduced pressure (Scheme 33$).{ }^{57}$

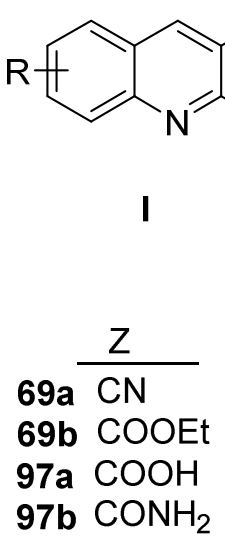<smiles>[X]CC#N</smiles>

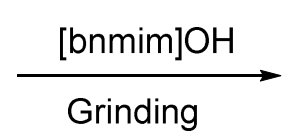

$69(a, b), 97 a, b$<smiles>[Z]/C(C#N)=C\c1cc2ccccc2nc1Cl</smiles>

$98 \mathrm{a}-0$

\begin{tabular}{llll}
\multicolumn{1}{c}{$\mathrm{R}$} & \multicolumn{2}{c}{$\mathrm{Z}$} & Yield (\%) \\
\cline { 2 - 4 } 98i & 7-Me, 8-Cl & $\mathrm{CONH}_{2}$ & 95 \\
98i $\mathrm{H}$ & $\mathrm{COOEt}$ & 92 \\
98k 7-OMe & COOEt & 90 \\
98I 6-Me & COOEt & 85 \\
98m 7-Me & COOEt & 92 \\
98n 8-Me & COOEt & 93 \\
980 6-Et & COOEt & 90
\end{tabular}

98i 7-Me, 8-Cl $\mathrm{CONH}_{2} 95$ 98i $\mathrm{H} \quad$ COOEt 92 98k 7-OMe COOEt 90 98I 6-Me COOEt 85 98m 7-Me 980 6-Et COOEt 90

\section{Scheme 33}

Description of the synthesis of novel 1,4-dihydropyrazolo-pyrano-[2,3-b]quinoline derivatives 99a-i, by reacting 2-chloroquinoline-3-carbaldehydes 1 with 3-substituted-1H-pyrazol-5(4H)-one 67a-c in refluxing ethanol mediated by $L$-proline was reported. This procedure was found efficient for various 2-chloroquinoline3-carbaldehydes la-e bearing electron donating and electron withdrawing groups (Scheme 34). ${ }^{45}$

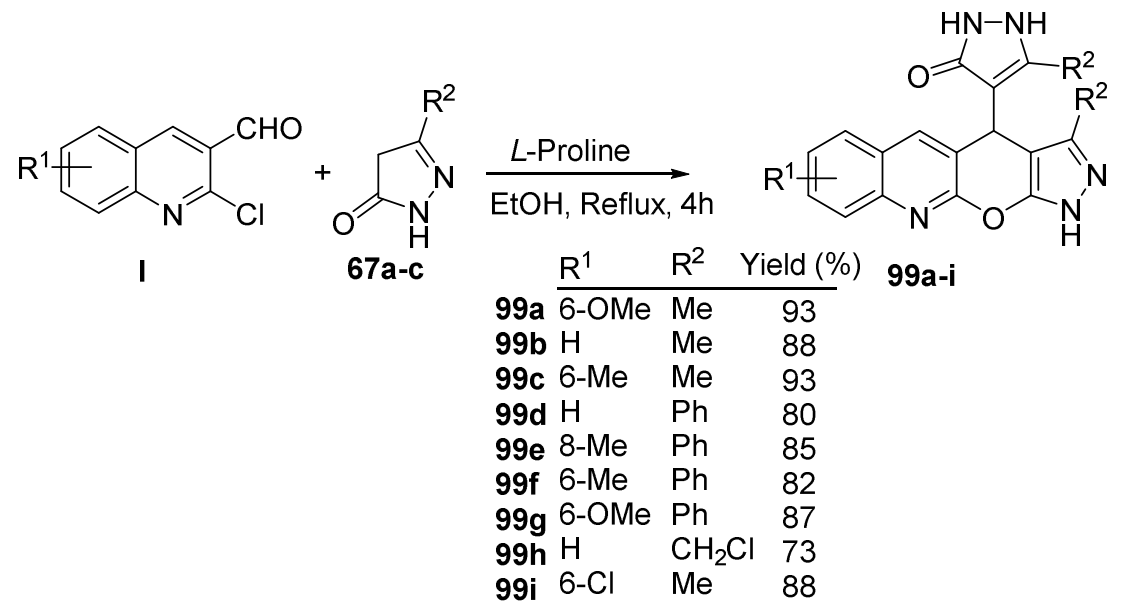

\section{Scheme 34}

The reaction of 2-chloroquinoline-3-carbaldehyde derivatives I and dimedone in the presence of KF$\mathrm{Al}_{2} \mathrm{O}_{3}$ to afford new pyranoquinolines 100a-d was reported. In this approach, a mechanism was proposed for the reaction course. Reasonable yields (41-50\%), easily available starting materials and less expensive efficient catalyst are the key features of this method (Scheme 35$).{ }^{58}$ 


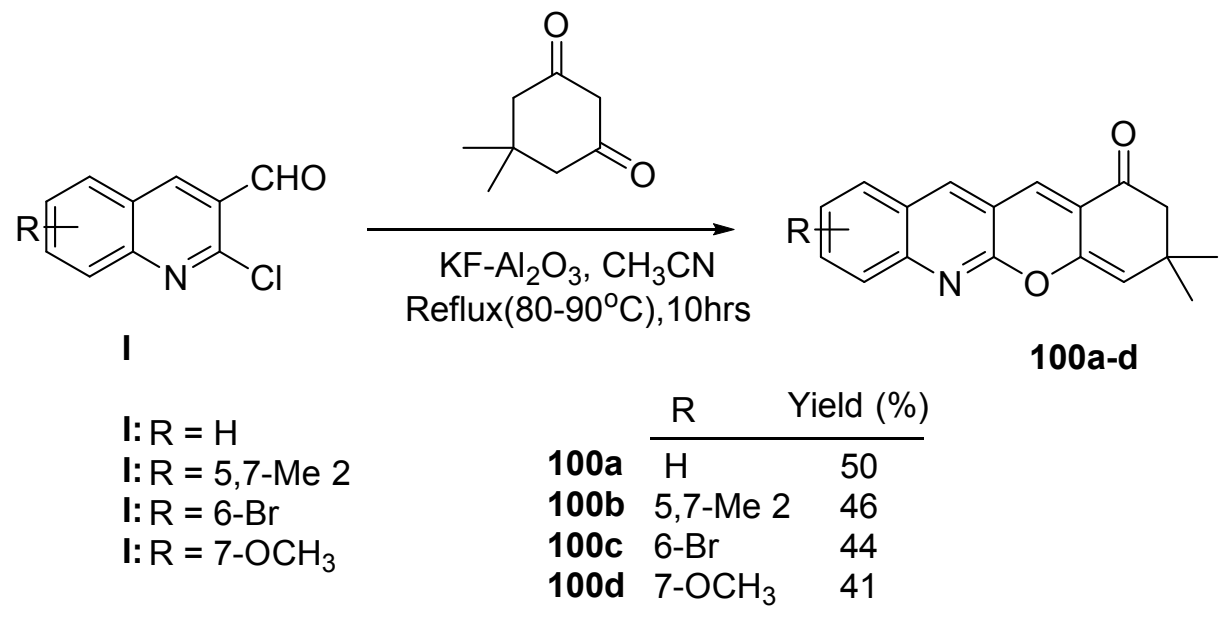

\section{Scheme 35}<smiles>[R]C=C1C=c2cc(C=O)c(Cl)nc2=C1</smiles>

I(1-9)

$\mathrm{I}(\mathrm{R})$

I(1): $\mathrm{H}$

I(2): $6-\mathrm{Me}$

I(3): $6-\mathrm{OMe}$

I(4): 6 -OEt

I(5): $5,7-\mathrm{Me} 2$

I(6): $7,8-\mathrm{Me} 2$

I(7): 6-t-butyl

I(8): 6-isopropyl I(9): 8-Et

$$
\text { 35( } \left.\mathrm{R}^{1}\right)
$$

35(1): $p-\mathrm{Me}$

35(2): H

35(3): $p$-OMe

35(4): $p-t$-butyl

35(5): $p-\mathrm{Cl}$

35(6): $p-\mathrm{Br}$

35(7): $p-\mathrm{NO}_{2}$

35(8): $p-\mathrm{F}, m-\mathrm{Cl}$

35(9): $m-\mathrm{Me} 2$

35(10): $\mathrm{o}-\mathrm{Cl}$

35(11): o-Et

35(12): benzo[2-3]

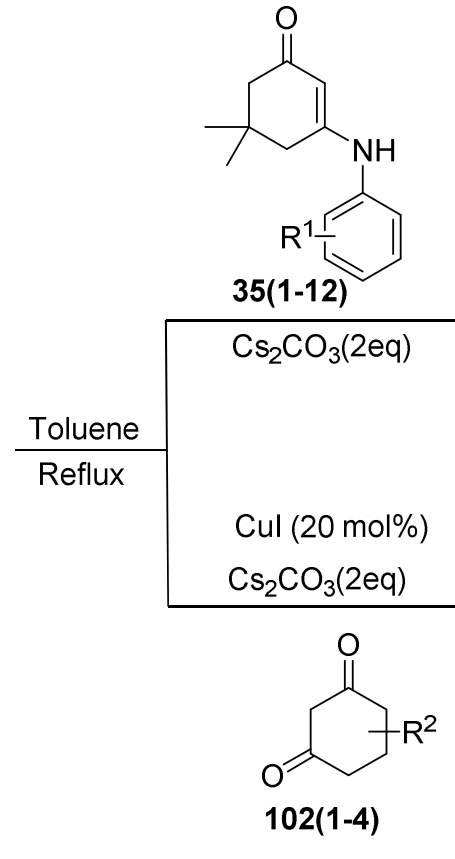

Entry $3\left(R, R^{1}\right)$ Yield(\%) Entry $3\left(R, R^{1}\right)$ Yield(\%)

\begin{tabular}{lll}
\hline 1 & $101(1,1)$ & 79 \\
2 & $101(1,2)$ & 80 \\
3 & $101(1,6)$ & 83 \\
4 & $101(1,7)$ & 84 \\
5 & $101(1,10)$ & 85 \\
6 & $101(1,12)$ & 83 \\
7 & $101(2,1)$ & 87 \\
8 & $101(2,8)$ & 82 \\
9 & $101(3,1)$ & 84 \\
10 & $101(3,2)$ & 85 \\
11 & $101(4,2)$ & 82 \\
12 & $101(4,3)$ & 76 \\
13 & $101(5,1)$ & 83 \\
14 & $101(6,1)$ & 84 \\
15 & $101(6,5)$ & 84
\end{tabular}

$\begin{array}{lll}16 & 101(7,1) & 87 \\ 17 & 101(7,2) & 82 \\ 18 & 101(7,3) & 85 \\ 19 & 101(7,4) & 86 \\ 20 & 101(7,5) & 78 \\ 21 & 101(7,6) & 75 \\ 22 & 101(7,7) & 82 \\ 23 & 101(7,8) & 85 \\ 24 & 101(7,9) & 84 \\ 25 & 101(7,11) & 83 \\ 26 & 101(7,12) & 82 \\ 27 & 101(8,1) & 84 \\ 28 & 101(8,2) & 83 \\ 29 & 101(8,7) & 82 \\ 30 & 101(9,9) & 84\end{array}$<smiles>[R]C=C1C2=Cc3cc4cc[R]([R1])cc4nc3N(c3cccc[R1]3[H])C2=CC1(C)C</smiles>

101(1-30)<smiles>[R]C/C=C1/Oc2nc3cc[R]([Y10])cc3cc2C=C1C(C)=O</smiles>

4( $\left.R^{2}\right) \quad$ Entry $5\left(R, R^{2}\right)$ Yield $(\%)$ 102(2): $5-\mathrm{Me} \quad 2 \quad 103(2,1)$ 102(3): $5-p \quad 3 \quad 103(3,1)$ 102(4): 5-propyl 4 103(4,1) 92

$\begin{array}{llll}5 & 103(5,1) & 89\end{array}$

$\begin{array}{lll}6 & 103(7,1) \quad 85\end{array}$

$\begin{array}{lll}7 & 103(8,1) & 94\end{array}$

$\begin{array}{lll}8 & 103(9,1) & 87\end{array}$

$\begin{array}{lll}9 & 103(7,2) & 81\end{array}$

$10 \quad 103(7,3) \quad 84$

\section{Scheme 36}


An efficient synthesis of novel functionalized 1,8-naphthyridine 101(1-30) and chromeno[2,3b]quinolines 103(1-10) derivatives via cascade reaction of 2-chloroquinoline-3-carbaldehyde $\mathbf{1 ( 1 - 9 )}$ and enaminones 35(1-12) or cyclic 1,3-dicarbonyl compounds 102(1-4) was introduced. The crude products of 101(1-30) were purified by recrystallization from $95 \%$ ethanol, while the crude products of 103(1-10) were purified by column chromatography to afford the pure products. All of the newly synthesized compounds were evaluated for their in vitro antiproliferative properties against cancer; several compounds were found to have high activities (Scheme 36). ${ }^{59}$

A synthetic route of novel highly substituted cyclopentadienes containing quinoline nucleus was described, in which a Knoevenagel adducts 104a-c of 2-chloroquinoline-3-carbaldehydes I and malononitrile or ethyl cyanoacetate were prepared. The reaction mixture was stirred in ethanol for 15 min at room temperature. After completion of the reaction, the solid was separated by filtration (Scheme 37 ). ${ }^{37}$

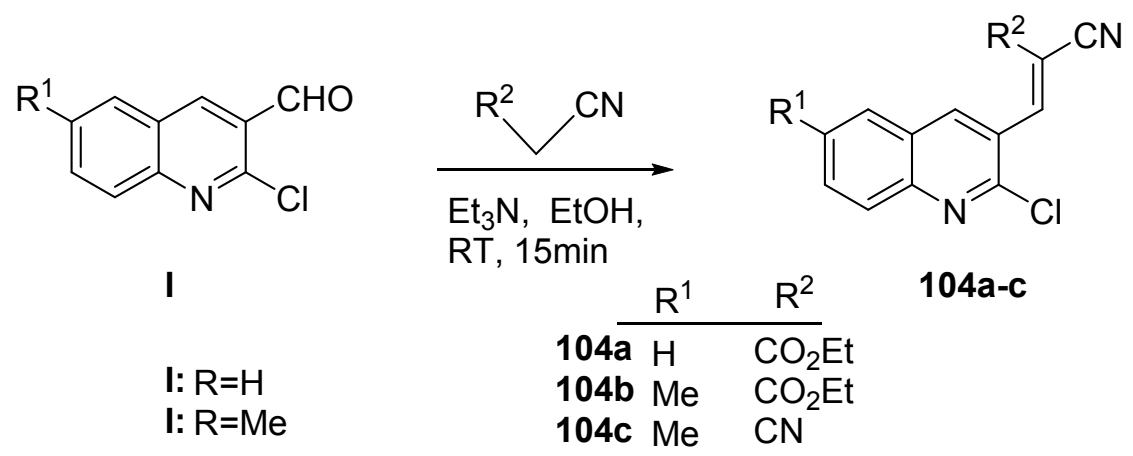

\section{Scheme 37}

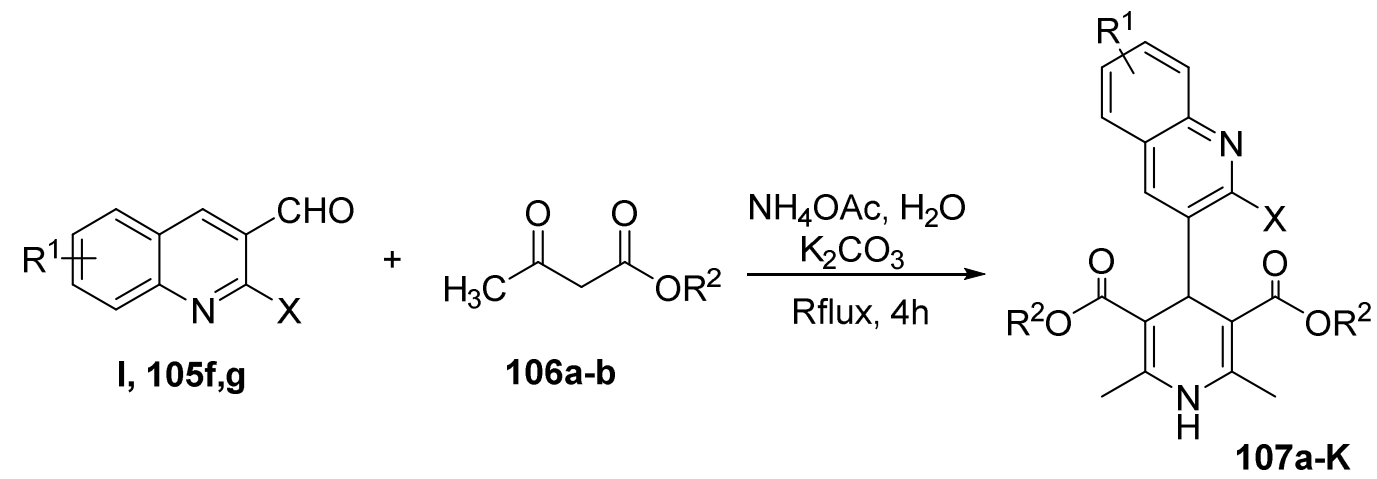

\begin{tabular}{|c|c|c|c|c|c|c|c|c|}
\hline \multirow{12}{*}{$\begin{array}{l}\text { I: } \\
\text { I: } \\
\text { I: } \\
\text { I: } \\
\text { I: } \\
105 f \\
105 g\end{array}$} & $\mathrm{R}^{1}$ & \multirow[t]{2}{*}{$x$} & \multirow{2}{*}{$\begin{array}{l}\text { 106a: Et } \\
\text { 106b: Me }\end{array}$} & \multirow{3}{*}{$\begin{array}{l}107 a \\
107 b\end{array}$} & $\mathrm{R}^{1}$ & \multirow{2}{*}{$\mathrm{R}^{2}$} & \multicolumn{2}{|c|}{$X$ Yield $(\%)$} \\
\hline & & & & & $\mathrm{H}$ & & $\mathrm{Cl}$ & 78 \\
\hline & G-Me & $\mathrm{Cl}$ & & & 6-Me & $\mathrm{Me}$ & $\mathrm{Cl}$ & 79 \\
\hline & 6-Me & $\mathrm{Cl}$ & & $107 c$ & $\mathrm{H}$ & $\mathrm{Me}$ & $\mathrm{Cl}$ & 82 \\
\hline & $\begin{array}{l}\text { o-Uivie } \\
\text { 8-Me }\end{array}$ & $\mathrm{Cl}$ & & $107 d$ & $6-\mathrm{OCH}_{3}$ & $\mathrm{Et}$ & $\mathrm{Cl}$ & 85 \\
\hline & 7-OMe & CI & & $107 e$ & $6-\mathrm{OCH}_{3}$ & $\mathrm{Me}$ & $\mathrm{Cl}$ & 88 \\
\hline & $\mathrm{H}$ & 1 & & $107 f$ & 8-Me & ET & $\mathrm{Cl}$ & 75 \\
\hline & & 1 & & $107 \mathrm{~g}$ & 8-Me & $\mathrm{Me}$ & $\mathrm{Cl}$ & 78 \\
\hline & & & & $107 \mathrm{~h}$ & $7-\mathrm{OCH}_{3}$ & $\mathrm{Me}$ & $\mathrm{Cl}$ & 84 \\
\hline & & & & $107 i$ & $\mathrm{H}$ & $\mathrm{Et}$ & I & 85 \\
\hline & & & & $107 j$ & $\mathrm{H}$ & $\mathrm{Me}$ & I & 83 \\
\hline & & & & $107 \mathrm{k}$ & $\mathrm{H}$ & $\mathrm{Me}$ & $\mathrm{O}$ & 87 \\
\hline
\end{tabular}

Scheme 38 
The synthesis of a novel series of substituted 1,4-dihydropyridines 107a-k was achieved in aqueous media by a base-catalyzed Hantzsch reaction of 2-chloroquinoline-3-carbaldehydes (I and 105f,g), ammonium acetate, and alkyl acetoacetate 106a-b in good to high yields was achieved (Scheme 38). ${ }^{60}$

Functionalized 2-amino-4H-benzo[b]pyran 42c and 108, dihydropyridine 107a, polyhydroquinoline 109, derivatives were synthesized at ambient temperature in aqueous medium, in the presence of $\mathrm{Bi}_{2} \mathrm{WO}_{6}(5 \mathrm{~mol}$ \%) as a catalyst. $\mathrm{Bi}_{2} \mathrm{WO}_{6}$ nanoparticle mediated multicomponent reactions (at $\mathrm{RT}$, in aq. medium) afforded good yields in a short period of time (10-45 min; 5 mol\% of catalyst) (Scheme 39). ${ }^{61}$

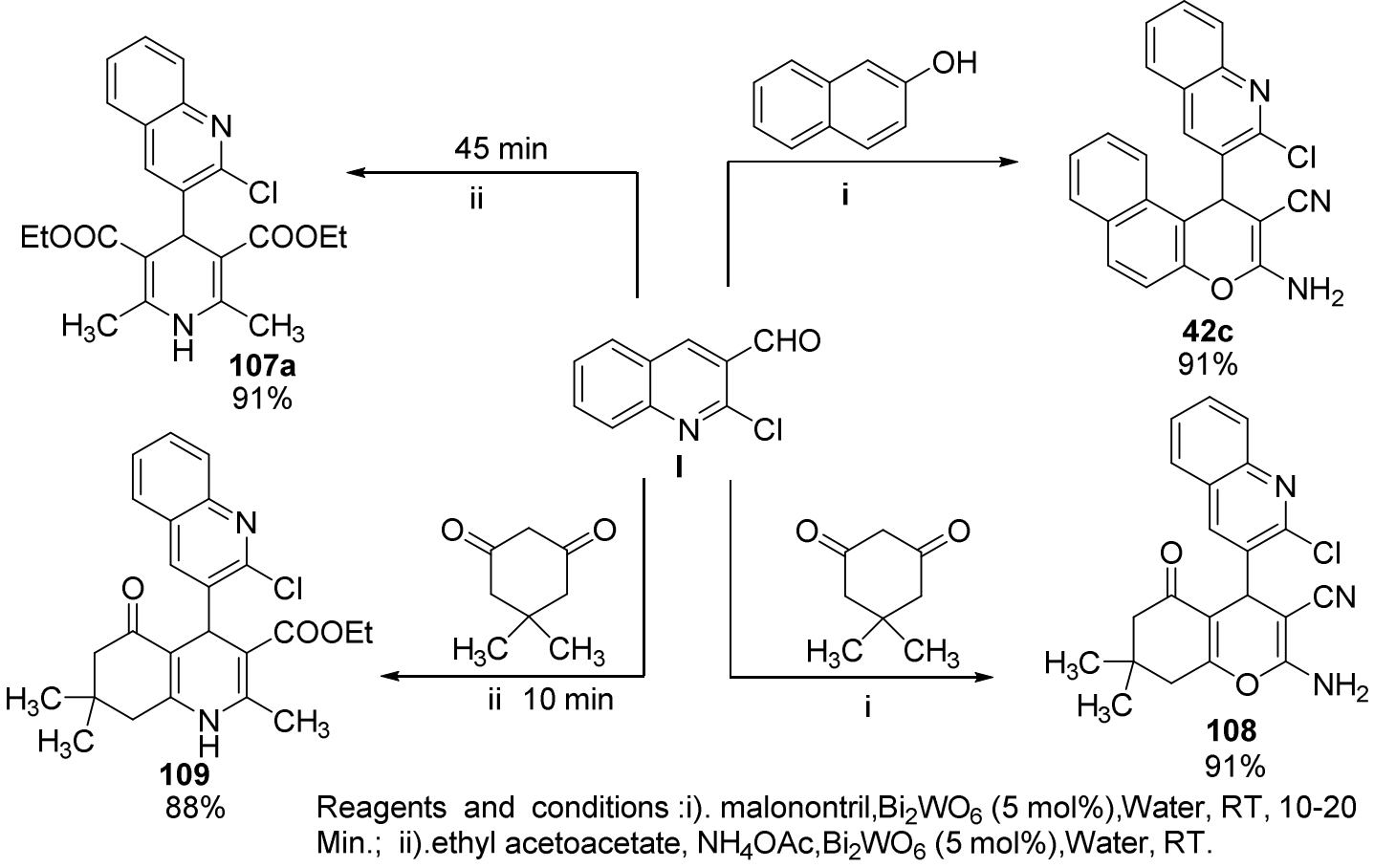

\section{Scheme 39}

Herein, thirteen new racemic, diversely functionalized 2-chloroquinolin-3-yl substituted Pyrano Tacrines (PTs) 111a-m were synthesized from the corresponding readily available $4 H$-pyrans and diverse commercial cycloalkanones, by using a Friedländer-type reaction ${ }^{62}$, under standard reaction conditions (Scheme 40). ${ }^{63}$ 

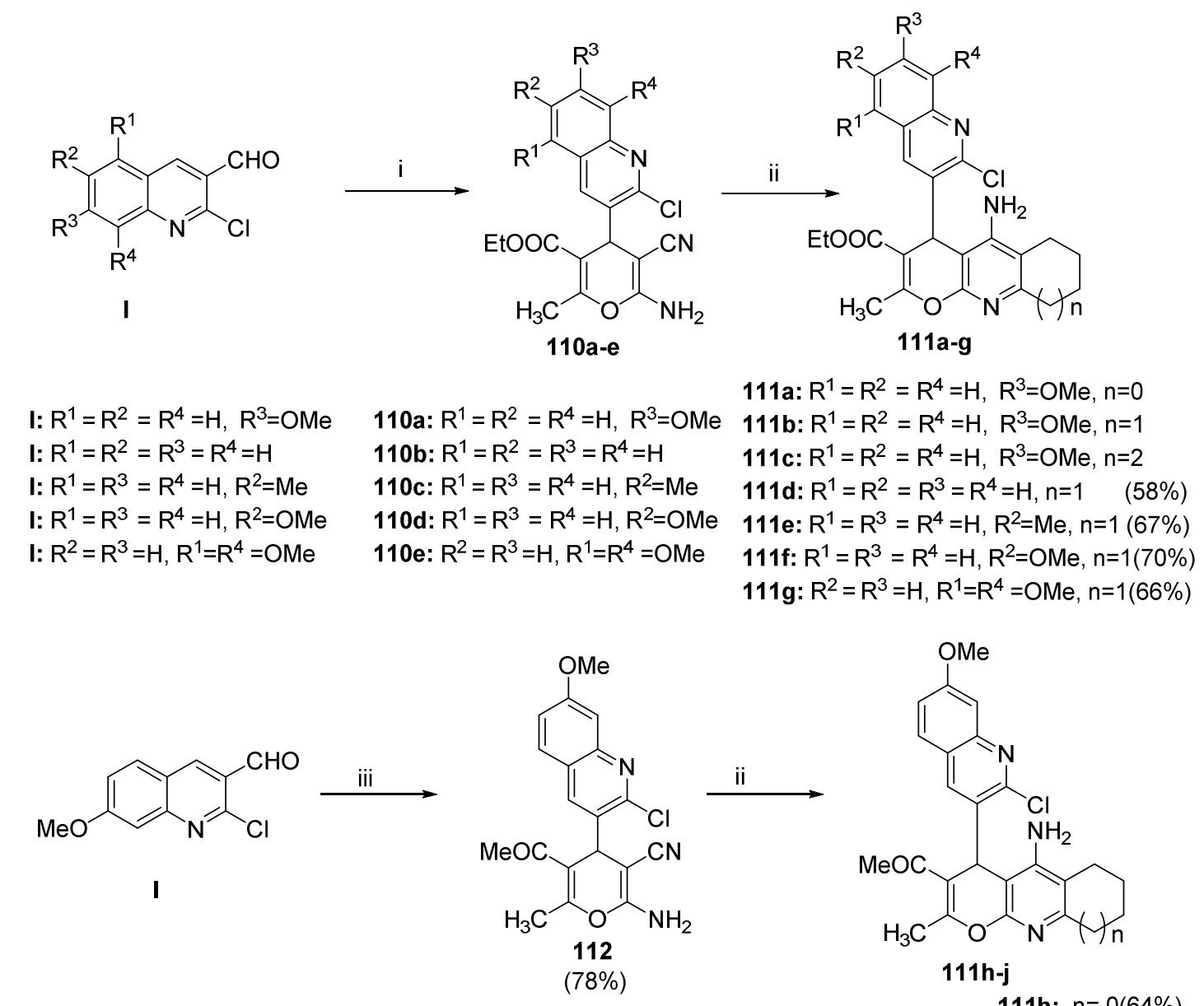

111h: $n=0(64 \%)$

111i: $n=1(66 \%)$
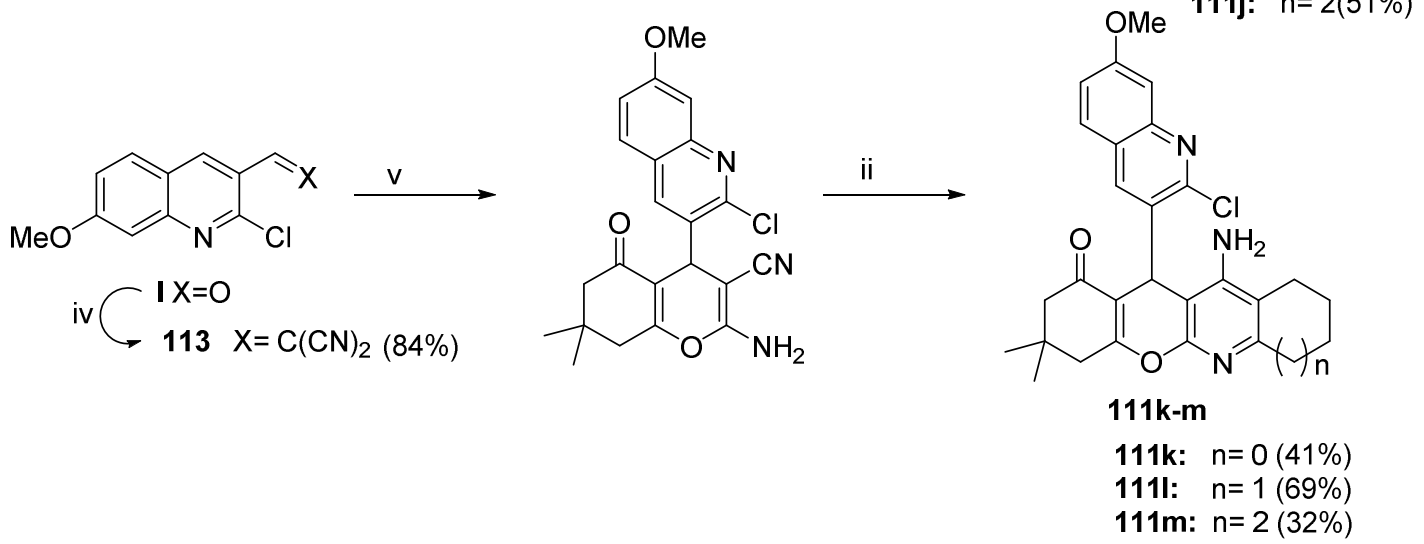

Reagents and conditions: i: a. $\mathrm{CNCH}_{2} \mathrm{CN}$, piperidine (cat.), $\mathrm{EtOH}, \mathrm{rt}, 20 \mathrm{~min}$; b. ethyl acetoacetate, piperidine(cat.); ii. Cycloalkanone, 1,2-dichloroethane, $\mathrm{AlCl}_{3}$, reflux; iii: a. $\mathrm{CNCH}_{2} \mathrm{CN}$, piperidine (cat.), $\mathrm{EtOH}$, rt, $20 \mathrm{~min}$; b. 2,4-pentanedione, piperidine (cat.); iv. $\mathrm{CNCH}_{2} \mathrm{CN}$, piperidine (cat.), EtOH, rt, 20 min; v: dimedone, $\mathrm{Et}_{3} \mathrm{~N}$ (cat.), $\mathrm{EtOH}, \mathrm{rt}, 1 \mathrm{~h}$.

\section{Scheme 40}

3.4.2. Reactions with hydrazine, hydroxylamine, hydrazides, (thio)semicarbazide, and urea. The synthesis of new series of quinolinyl Schiff's bases and azetidinones was reported. The aldehydes I, upon treatment with methanol in the presence of $\mathrm{KOH}$ furnished the corresponding 2-methoxyquinoline-3-carbaldehydes 114a-d. The Schiff's bases 115a-d and 116-d were prepared by reacting 114a-d with isoniazid and 4-(1H-pyrrol-1yl)benzohydrazide in the presence of glacial acetic acid, respectively. While the Schiff's bases 117a-d were 
prepared by reacting I with isoniazid in ethanol in the presence of glacial acetic acid. Compounds 115a-d, 116a-d, and 117a-d upon treatment with monochloroacetyl chloride in the presence of TEA in dry benzene gave 118a-d, 119a-d, and 120a-d, respectively (Scheme 41). ${ }^{64}$

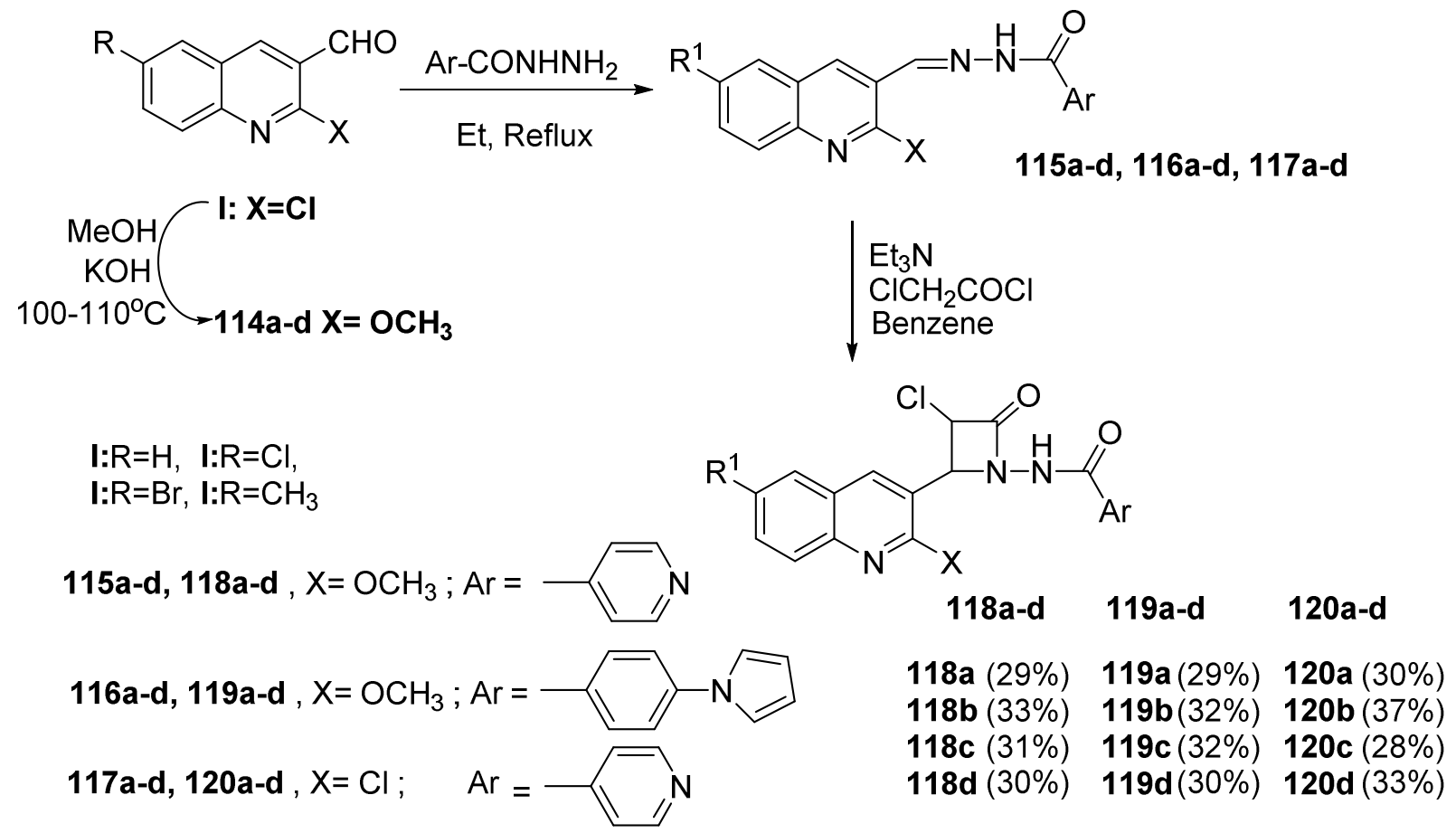

\section{Scheme 41}

A new series of quinoline derivatives 122a-g were synthesized by refluxing a mixture of 2chloroquinoline-3-carbaldehydes I, 1,1-dimethylhydrazine 121, few drops of glacial acetic acid in EtOH for $4 \mathrm{~h}$. After completion of the reaction, distilled water was added to the reaction mixture, the resulting solid was separated by filtration, and recrystallized from ethanol to afford pure products (Scheme 42). ${ }^{65}$<smiles>O=Cc1cc2ccccc2nc1Cl</smiles>

I<smiles>[R10][R10]([H])(OC)C(=O)O</smiles>

121<smiles>CN(C)/N=C/c1cc2cc[R]cc2nc1Cl</smiles>

122a-g

\begin{tabular}{|c|c|c|}
\hline & $\mathrm{R}$ & Yield (\%) \\
\hline $122 a$ & & 70 \\
\hline $122 b$ & 6-Me & 72 \\
\hline $122 c$ & 6-OMe & 75 \\
\hline 122d & 6-Cl & 69 \\
\hline $122 \mathrm{e}$ & 7-Me & 80 \\
\hline $122 f$ & 6-isopropyl & yl 70 \\
\hline $122 \mathrm{~g}$ & $7-\mathrm{Cl}$ & 69 \\
\hline
\end{tabular}

\section{Scheme 42}

The synthesis of 2-chloroquinoline-3-carbaldehyde phenyl hydrazone derivatives 124a-k by two methods was described. The first method is in solution, by stirring substituted aldehyde I and substituted 
phenyl hydrazine 123a-c in $\mathrm{MeOH}$ at room temperature over 2-15 h, while the second method is in solid state by grinding reactants to form products in short time (Scheme 43 ). ${ }^{66}$

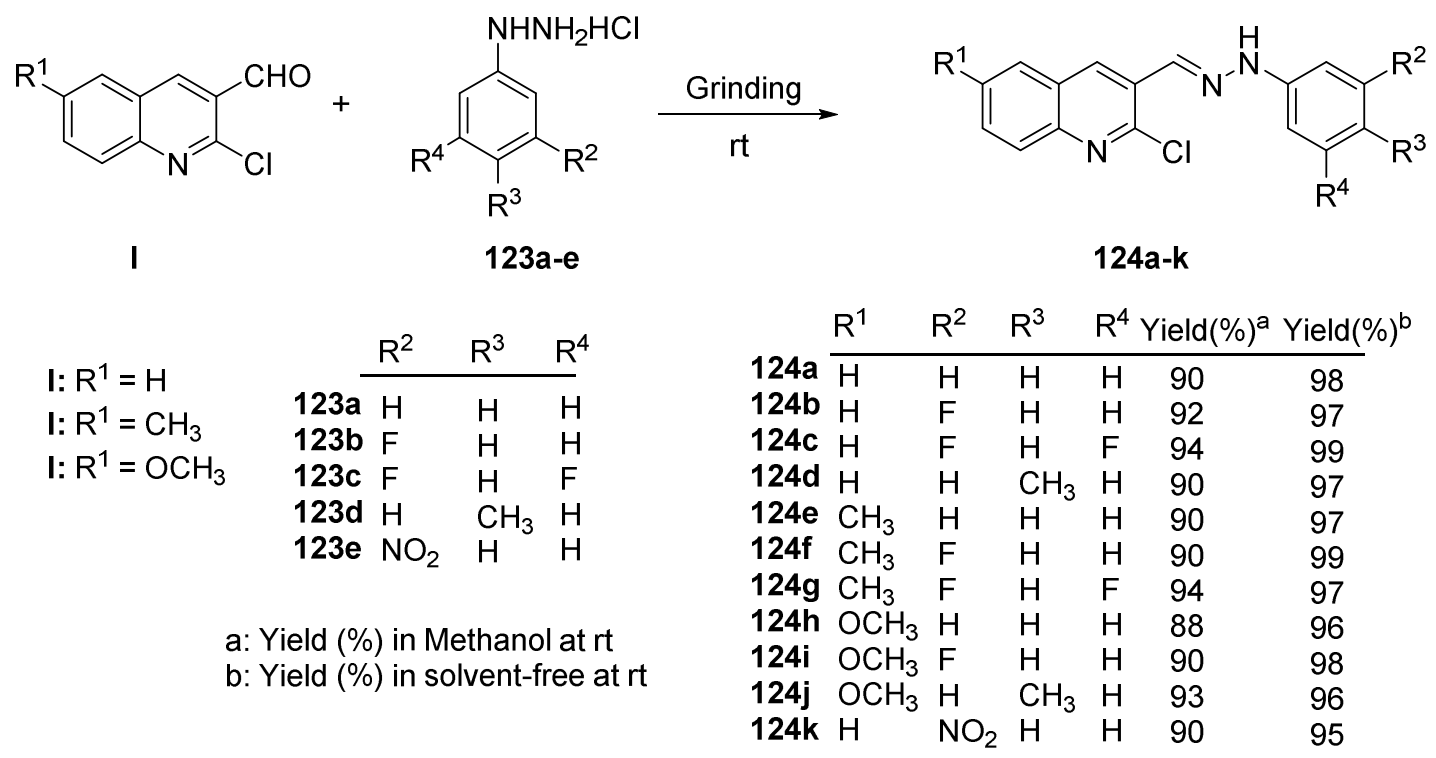

\section{Scheme 43}

Dry grinding of a mixture of the aldehydes I and 4-methylphenylhydrazinium chloride 123d afforded the hydrazone derivative $\mathbf{1 2 5}(\mathbf{A}, \mathbf{B})$, while the same reagents in methanol in the presence of sodium cyanoborohydride gave the $1 H$-pyrazolo[3,4-b]quinoline 127 a (Scheme 44$).{ }^{66,67}$
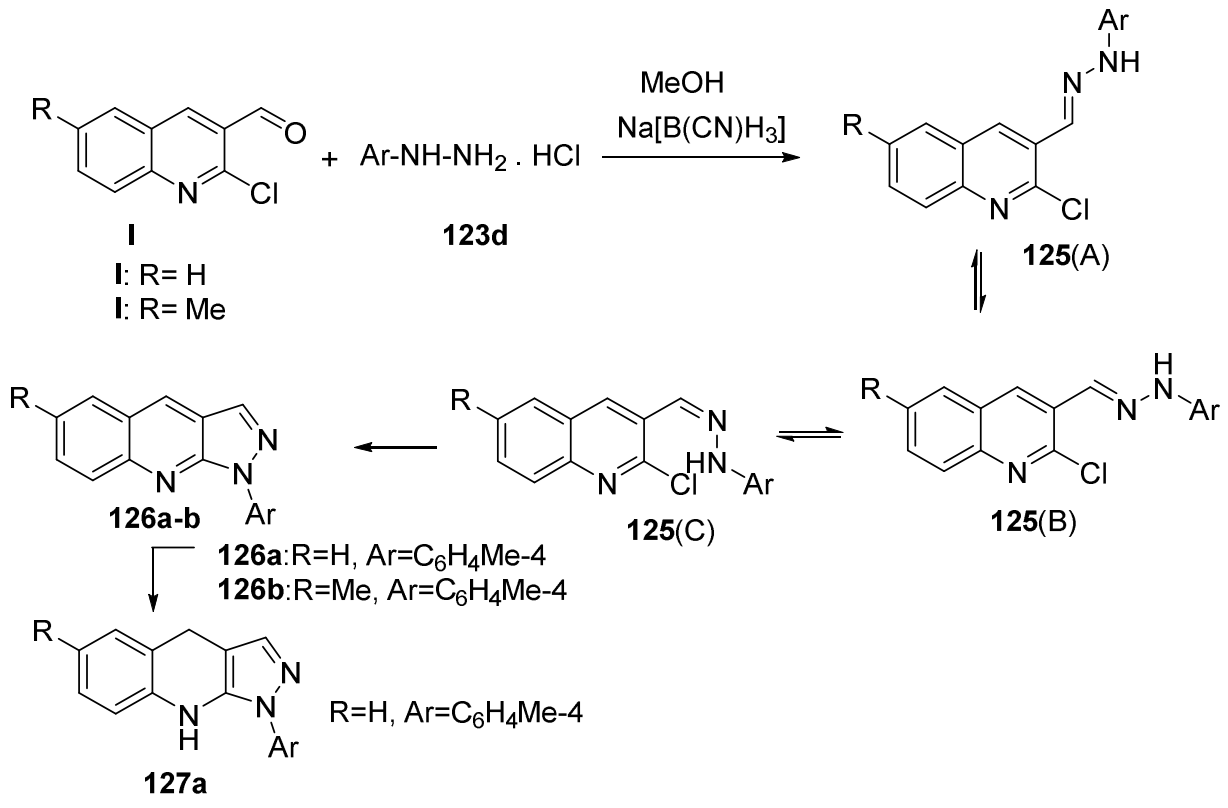

\section{Scheme 44}

On refluxing the benzimidazolylacetohydrazides $128 \mathrm{a}-\mathrm{c}$ with I in ethanol, the acetohydrazides $129 \mathrm{a}-\mathrm{c}$ were afforded. 1,3,4-Oxadiazoles 130a-c were prepared by refluxing $N^{\prime}$-((2-chloroquinolin-3-yl)methylene)-2(2-substituted-1H-benzo[d] imidazol-1-yl)acetohydrazide 129a-c with chloramine-T in ethanol (Scheme 45). ${ }^{8,68}$ 


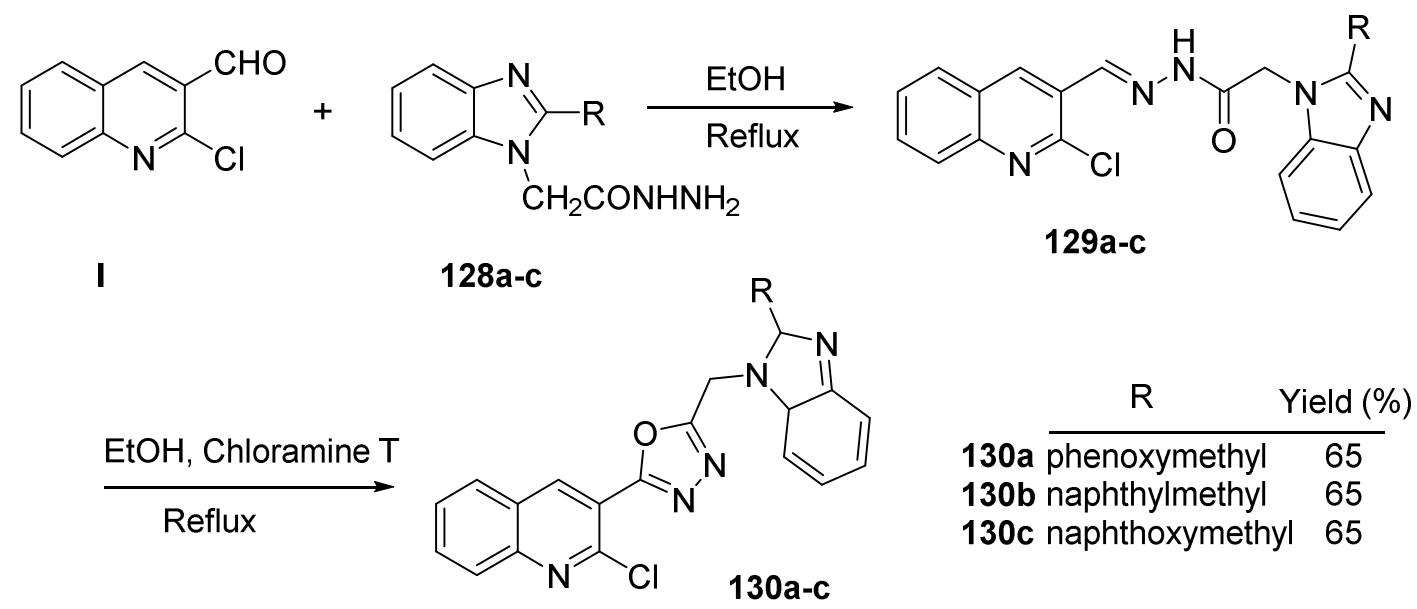

\section{Scheme 45}

Similarly, the different hydrazides 132a-i were synthesized starting from aromatic acids 131a-i. The benzohydrazide derivatives $\mathbf{1 3 3 a - i}$ were synthesized by reacting the aldehyde I and the appropriate hydrazide 132a-i. In the final step, the oxadiazol-2-ylquinolines 134a-i were synthesized with chloramine-T as aforementioned (Scheme 46). ${ }^{68,69}$

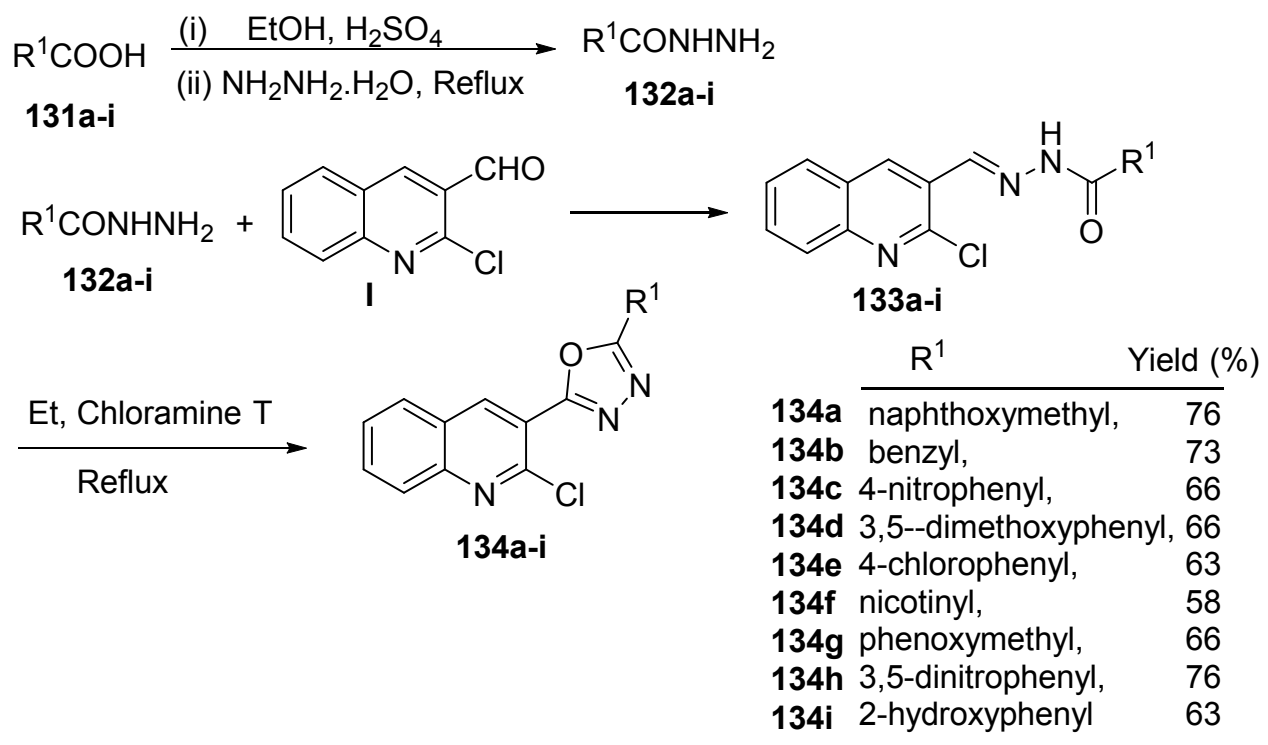

\section{Scheme 46}

2-Oxo-1,2-dihydroquinoline-3-carbaldehyde 135 was obtained by refluxing 2-chloroquinoline-3carbaldehyde $\mathbf{I}$ in $70 \%$ acetic acid. While 2-(p-tolyloxy)quioline-3-carbaldehyde 136 was prepared by refluxing 135 with $p$-cresol in DMF and using catalytic amount of $\mathrm{K}_{2} \mathrm{CO}_{3}$ (Scheme 47$){ }^{8}$<smiles>O=Cc1cc2ccccc2nc1Cl</smiles>

I

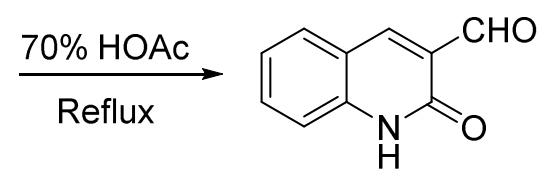

135

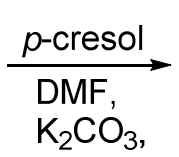

Reflux<smiles>Cc1ccc(OC2Nc3ccccc3C=C2C=O)cc1</smiles> 
2-Oxo-1,2-dihydroquinoline-3-carbaldehyde 135 was used for preparation of the series of 2,5disubstituted 1,3,4-oxadiazoles 139-140 and 149-156. This series were synthesized in search of potential therapeutics for cancer. The corresponding 1,3,4-oxadiazoles has been synthesized by chloramine-T and refluxing in ethyl alcohol. (Scheme 48$).^{8}$

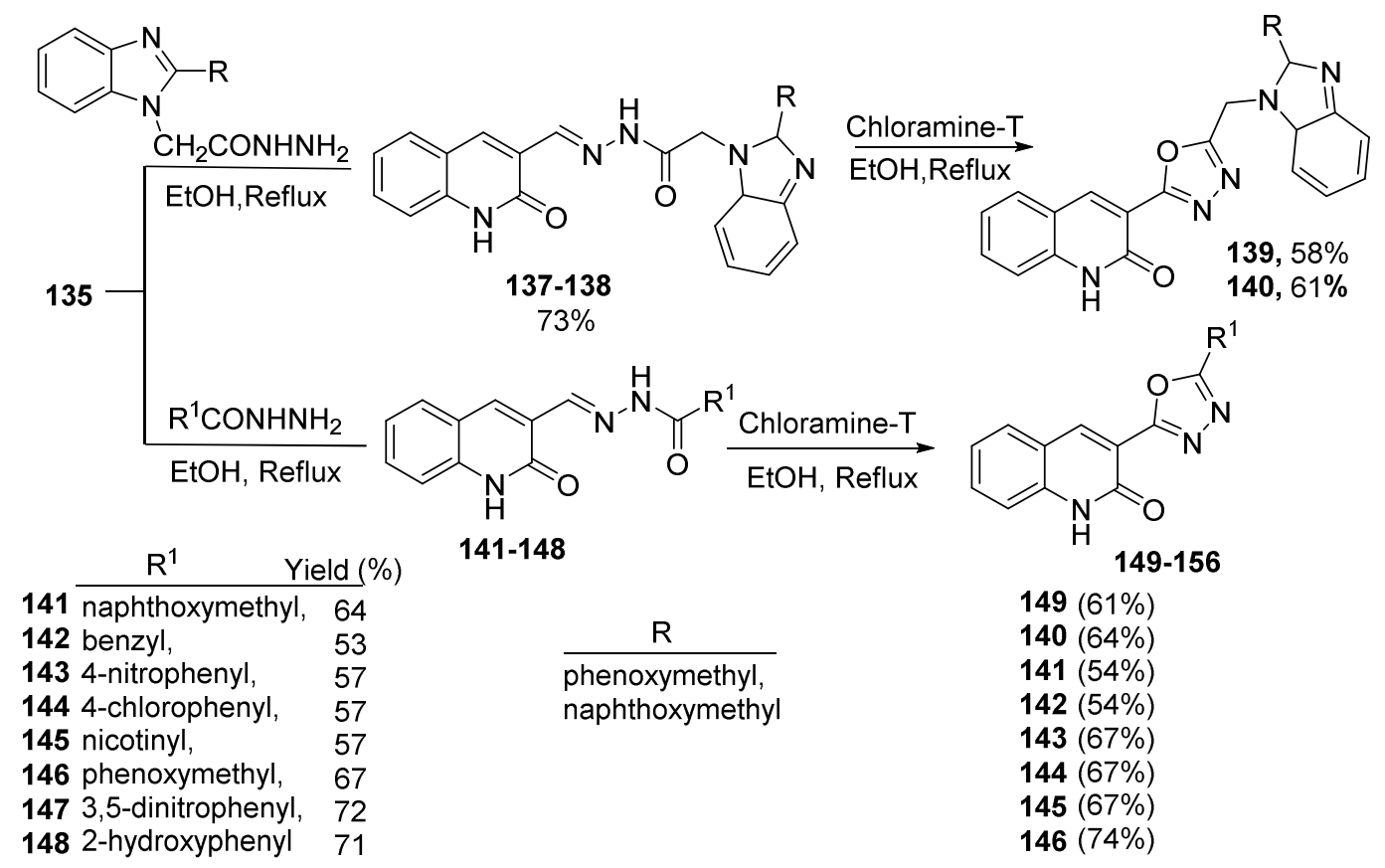

\section{Scheme 48}

For the synthesis of the second series of 2,5-disubstituted 1,3,4-oxadiazoles 159-160, 169-176, 2-(ptolyloxy)quioline-3-carbaldehyde 136 was used as a starting aldehyde. This series had also been synthesized using chloramine-T and refluxing in ethanol. The study revealed that compound $\mathbf{1 5 9}$ is a potent lead compound for anticancer drug discovery (Scheme 49). ${ }^{8}$

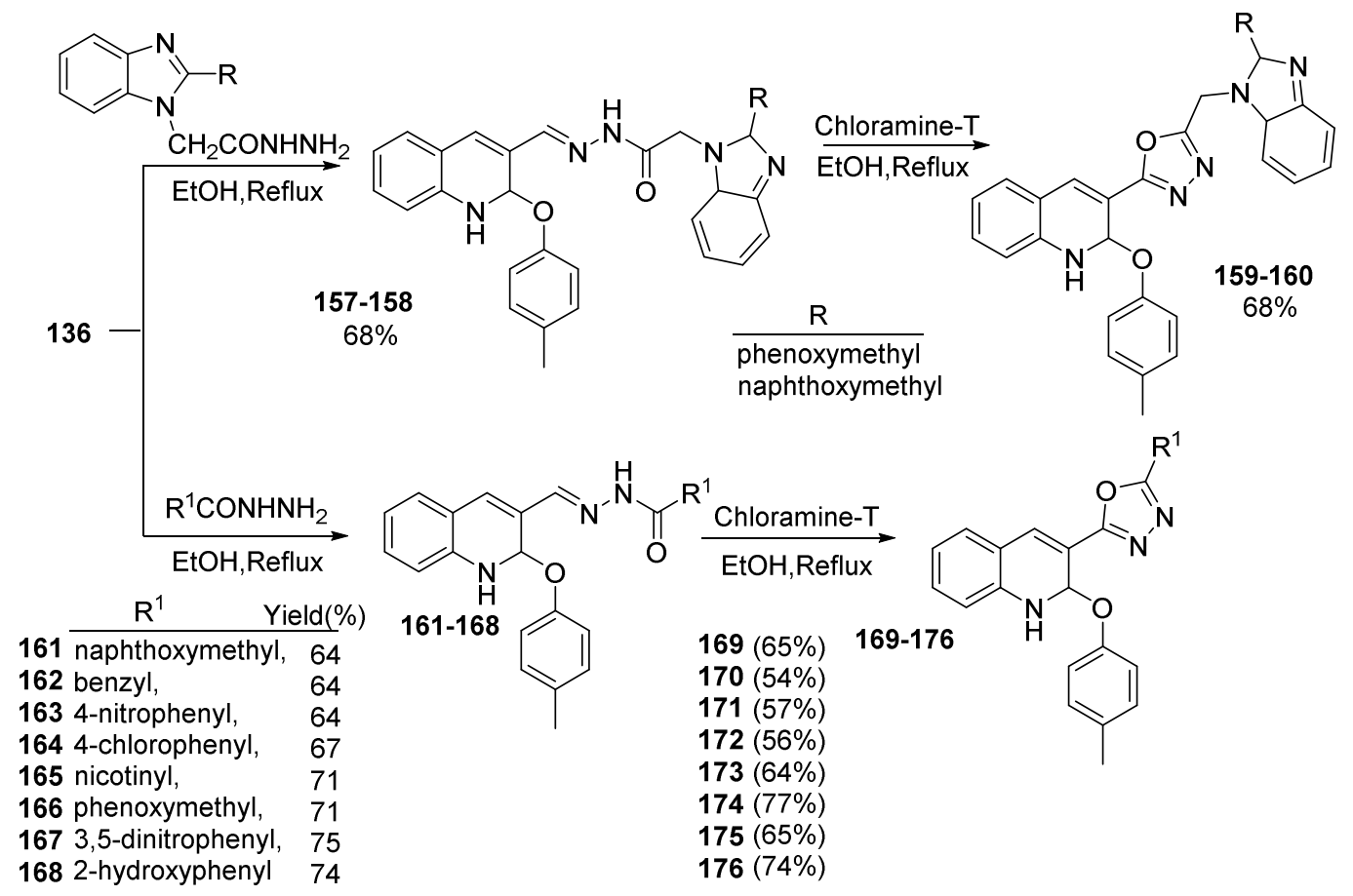

\section{Scheme 49}


A procedure was described for the synthesis of 3-(2-chloroquinolin-3-yl)-5-phenylisoxazoles 178a-j. The appropriate aldehyde I was added to a hydroxylamine solution in ethanol to afford the oxime 177a-g, which upon reaction with chloramine- $\mathrm{T}$ trihydrate, $\mathrm{CuSO}_{4} .5 \mathrm{H}_{2} \mathrm{O}, \mathrm{Cu}$ and phenylacetylene afforded the corresponding phenylisoxazoles 178a-j (Scheme 50). ${ }^{7}$

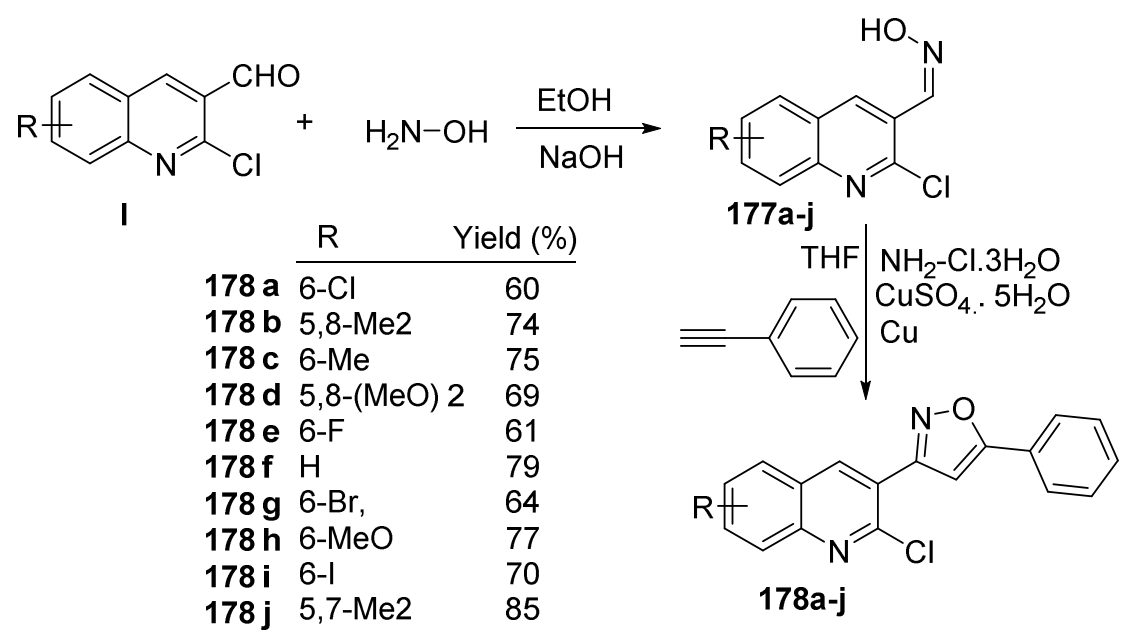

\section{Scheme 50}

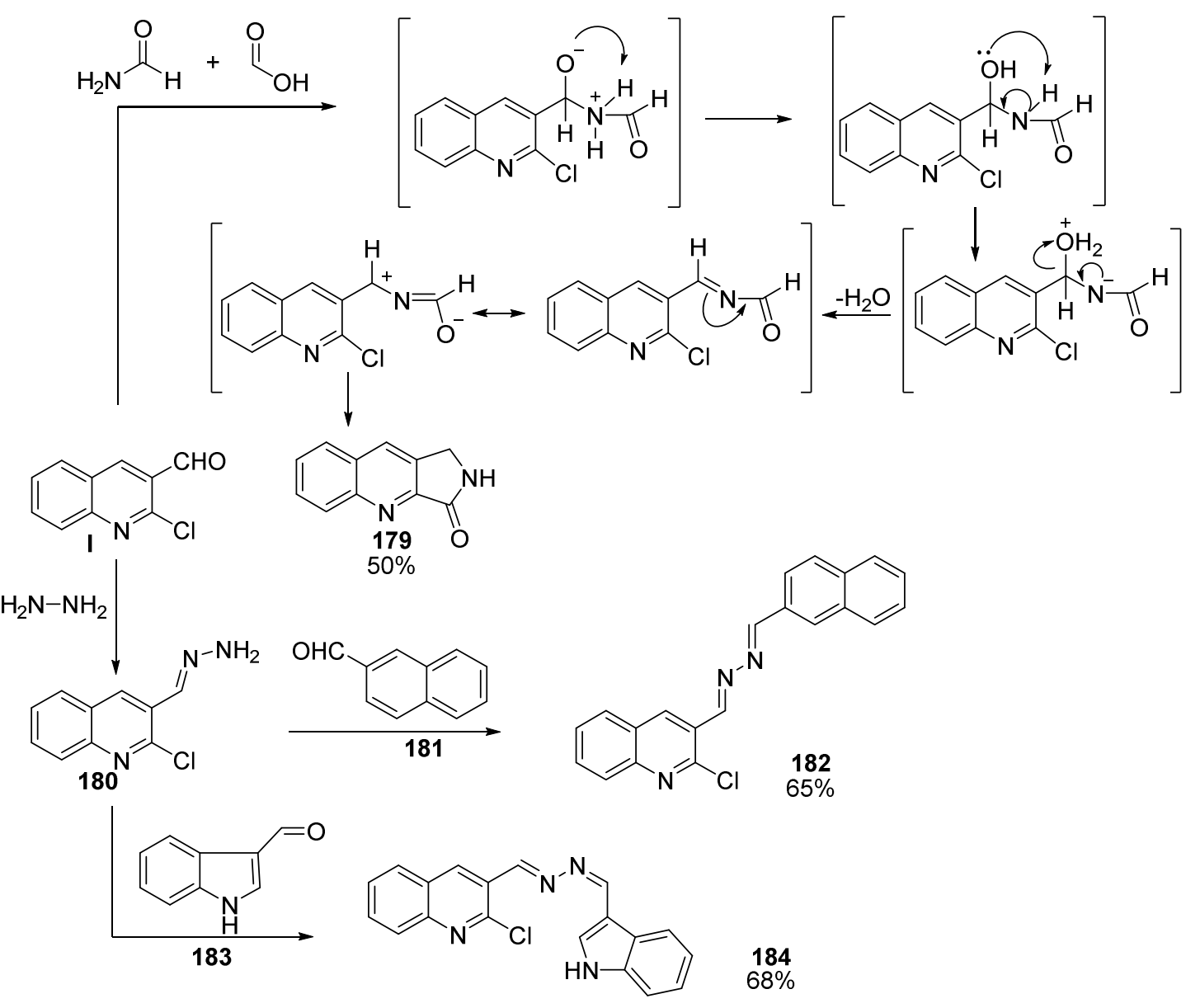

\section{Scheme 51}


When carbaldehyde I was treated with formamide and formic acid, the pyrrolo[3,4- $b]$ quinolin-3-one 179 was obtained, the reaction occurs via Leuckart reaction ${ }^{70}$ by using formamide as a formylating agent. On treating I with hydrazine hydrate, the hydrazone $\mathbf{1 8 0}$ was afforded, which then treated with aromatic aldehydes 181 and 183 to give 3-((naphthalen-2-yl/1H-indol-3-yl)methylene)hydrazono)methyl)quinoline 182 and 184, respectively (Scheme 51). ${ }^{40}$

Furthermore, the carbaldehyde group in I was transformed to a nitrile group via condensation reaction with hydroxylamine hydrochloride and sodium acetate to afford oxime $\mathbf{1 8 5}$. Dehydration of the aldoxime 185 with thionyl chloride gave the cyanoquinoline 186. The cyanoquinoline 186 was reacted with lithium aluminium hydride and potassium sodium tartarate in THF afforded the corresponding amine 187. In addition, cyclization of 186 with hydrazine hydrate afforded 188. Compound 190 was obtained from the reaction of 188 with benzoylisocyanate 189. In the same manner pyrazoloquinoline Schiff's base derivatives 191 and 192 were obtained in moderate yield by condensation reaction of 188 with naphthaldehyde 181 or indol-3-aldehyde 183 (Scheme 52). ${ }^{40}$

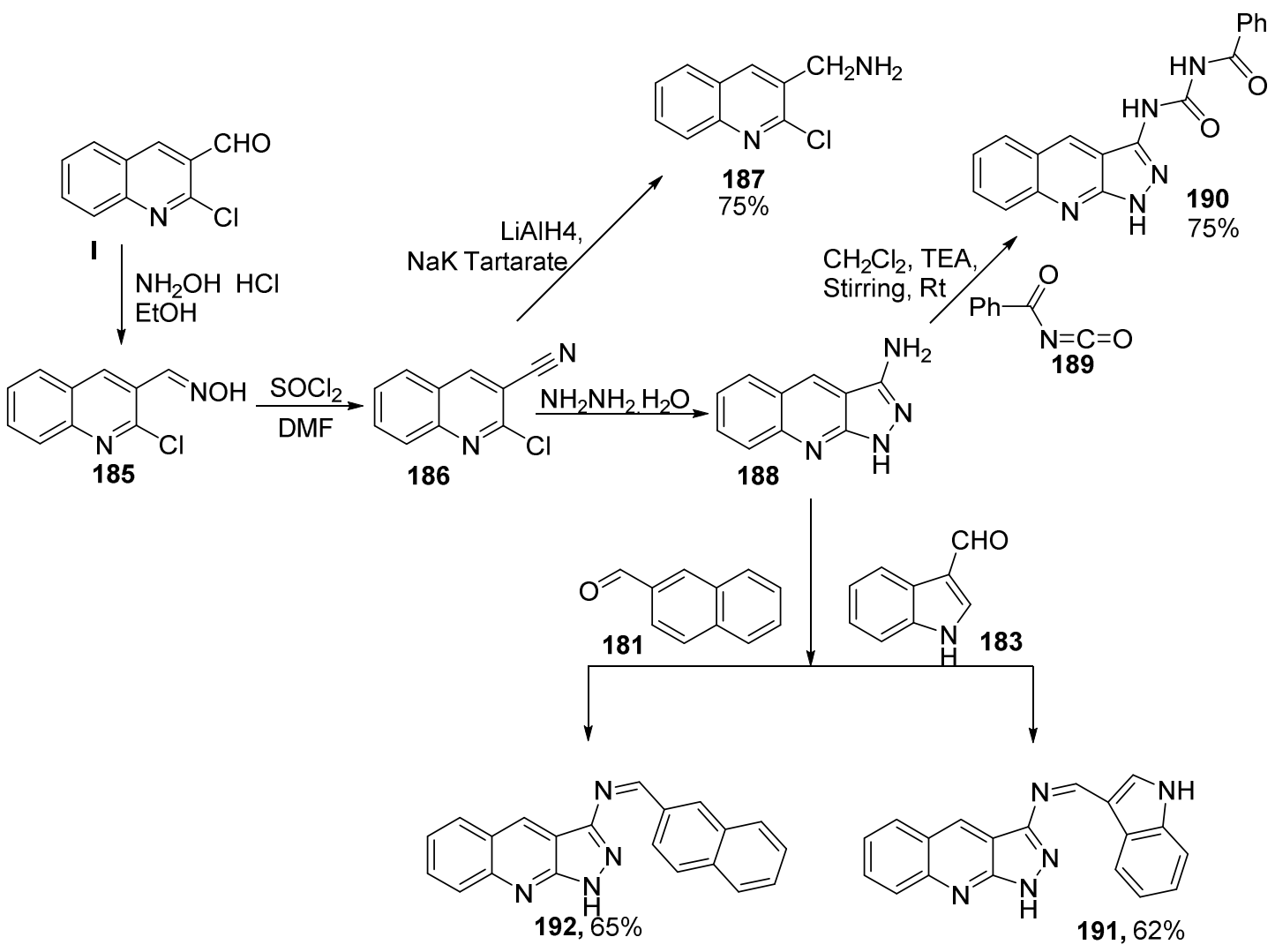

Scheme 52

3.4.3. Reactions with amines and amides. 2-Chloroquinoline-3-carbaldehyde I was reacted with amines 193ac to form an imine intermediate, which was subsequently cyclized to afford tetracyclic thio- and oxazepino derivatives 194a-d. ${ }^{71-73}$ While benzo[2,3][1,4]thia- or oxazepino[7,6- $b$ ]quinolones 195a-i was afforded by reacting carboxylic acid, isocyanide and 194a-d in methanol. The reaction mixture was stirred for $48 \mathrm{~h}$ at room temperature (Scheme 53). ${ }^{74}$ 


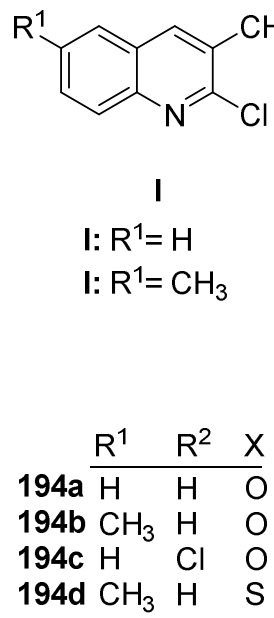<smiles>[X]c1ccc([R])c(N)c1</smiles>

193a-c

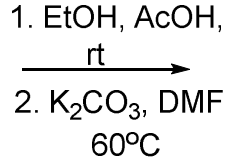

$60^{\circ} \mathrm{C}$<smiles>[R]c1ccc([X])c(/N=C\c2cc3cc([R1])ccc3nc2[X])c1</smiles>

194a-d

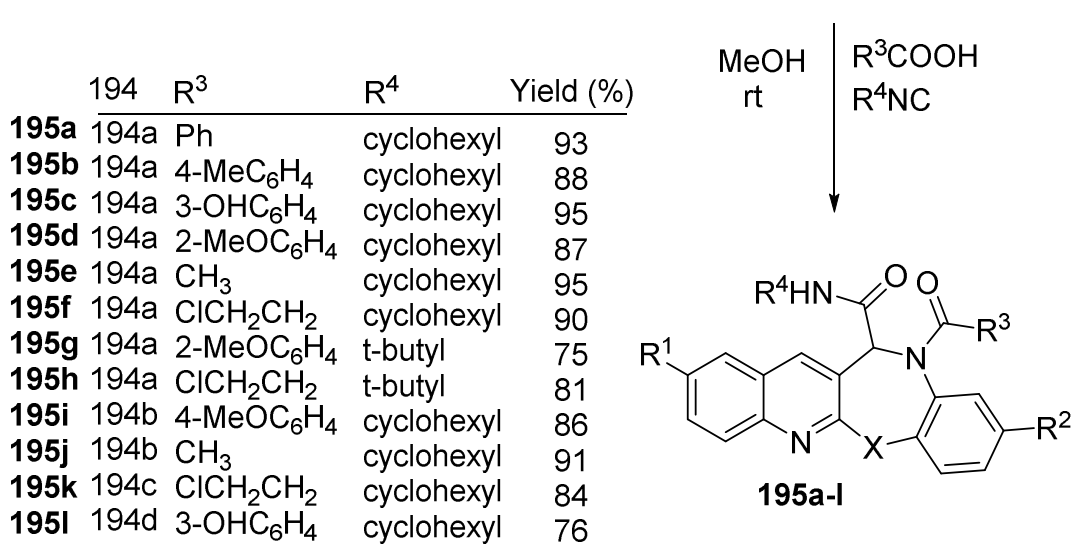

\section{Scheme 53}

Formation of mono and di Schiff's bases derivatives 197a-i derivatives was reported. The reaction took place between 2-chloroquinoline-3-carbaldehydes I and benzene-1,4-diamine 196 in ethanol under reflux. The reaction was catalyzed with acetic acid (Scheme 54$).^{1}$<smiles>CCc1cc2c(nc1Cl)C=C[R]C=C2</smiles>

I<smiles>Nc1ccc(N)cc1</smiles>

196

\begin{tabular}{|c|c|c|c|c|c|}
\hline & $\mathrm{R}$ & ield $(\%)$ & & $\mathrm{R}$ & $\operatorname{rield}(\%)$ \\
\hline $197 \mathbf{a}$ & $\mathrm{H}$ & 72 & $197 f$ & 7-Me & 70 \\
\hline 197b & 6-Me & 70 & $197 \mathrm{~g}$ & $6-\mathrm{Et}$ & 69 \\
\hline 197c & 6-OMe & 69 & $197 \mathrm{~h}$ & 6-Isopropyl & 68 \\
\hline 197d & 6-OEt & 68 & $197 \mathbf{i}$ & $7-\mathrm{Cl}$ & 75 \\
\hline $197 \mathrm{e}$ & $6-\mathrm{Cl}$ & 74 & & & \\
\hline
\end{tabular}

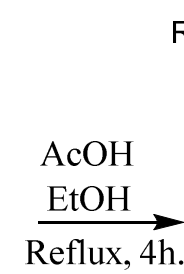

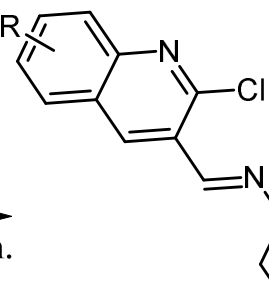

$197 \mathbf{a}-\mathbf{i}$<smiles>[R]c1ccc2nc(Cl)c(/C=N/c3ccccc3)cc2c1</smiles>

\section{Scheme 54}

Derivatives of novel pyrimido $\left[4^{\prime}, 5^{\prime}: 2,3\right][1,4]$ thiazepino[ $[7,6-b]$ quinoline ring 203a-f system have been synthesized through cyclocondensation of 5-amino-6-methylpyrimidine-4-thiols 202a,b and 2-chloroquinoline3-carbaldehydes I in the presence of $\mathrm{K}_{2} \mathrm{CO}_{3}$ in DMF (Scheme 55). ${ }^{75}$

Synthesis of a series of 23 novel unsymmetrical bis-heterocycles having either imidazo[2,1-b]thiazoles 207a-k or benzo[ $d$ ] imidazo[2,1-b]thiazole 209a-I frameworks bound with chromone, quinoline or julolidine in good to excellent yields by an acid-free Groebke-Blackburn-Bienaymé reaction (GBBR) under microwaveheating conditions, was reported (Scheme 56). ${ }^{76}$ 
<smiles>CC#CCNc1nc2c([N+](=O)[O-])nc(Cl)nc2s1</smiles>

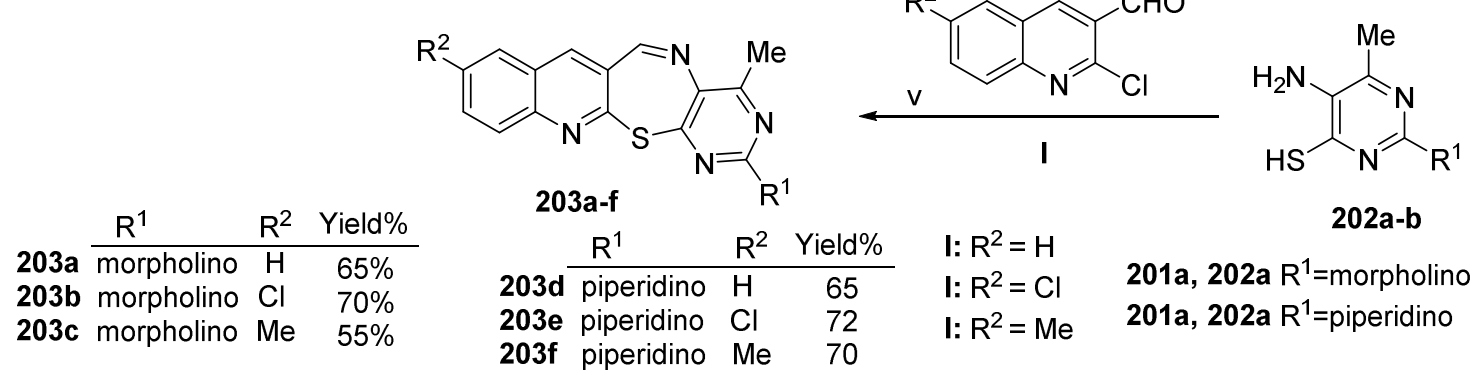

Reagents and conditions: (i) Fe powder, HOAc, rt, $2 \mathrm{~h}$; (ii) $\mathrm{KSCN}$, DMF, reflux, $3 \mathrm{~h}$; (iii) morpholine or piperidine, $\mathrm{EtOH}$, reflux, $6 \mathrm{~h}$; (iv) $\mathrm{KOH}(\mathrm{aq})$, reflux, $10 \mathrm{~h}$; (v) $\mathrm{K}_{2} \mathrm{CO}_{3}$, DMF, reflux, 8-12 h.

\section{Scheme 55}<smiles></smiles>

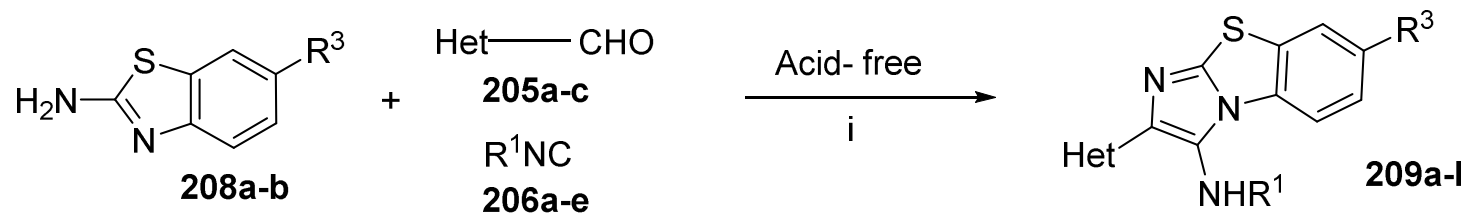

$\mathrm{i}=\mathrm{PhMe}, \mathrm{MW}\left(150 \mathrm{~W}, 150^{\circ} \mathrm{C}\right), 10 \mathrm{~min}$

$\mathrm{R}^{1}=t-$ But, c-Hex, Bn, 4-OMeBn, 3,4-diOMePhEt; $\mathrm{R}^{2}=\mathrm{H}, \mathrm{CN}, \mathrm{R}^{3}=\mathrm{H}, \mathrm{F}$

Het $=$<smiles>Cc1coc2ccccc2c1=O</smiles><smiles>Cc1cc2ccccc2nc1Cl</smiles><smiles>CC1=CC2CCCN3CCCC(=C1)C23</smiles>

$204 R^{2}$ 205(Het) 206 ${ }^{2}$

207a $\mathrm{H}$ Chromone $t$-Bu

207b H Chromone c-Hex

\section{7c H Chromone Bn}

207d H

207e H

$207 f \quad H$

$207 \mathrm{~g} \mathrm{H}$
Chromone 4-OMeBn

Chromone 3,4-diOMePhEt - 92

Quinoline $t-B u$

Quinoline c-Hex

$t$-Bu

$\mathrm{C}-\mathrm{Hex}$

$t-\mathrm{Bu}$

c-Hex
208R $\mathrm{R}^{3}$ Yield(\%)

- 96

- 95

- 93

$-91$

\begin{tabular}{l}
-92 \\
$-\quad 96$ \\
\hline
\end{tabular}

- $\quad 95$

- 92

- 90

- 84

- 82
$204 R^{2}$ 205(Het) 206 $R^{2}$

208R $\mathrm{R}^{3}$ Yield(\%)

209a - Chromone $t$-Bu

209b - Chromone c-Hex

$\mathrm{H} \quad 97$

209c - Chromone Bn

H 96

209d - Chromone 4-OMeBn

$\mathrm{H} \quad 92$

209e - Chromone 3,4-diOMePhEt H 90

$209 f$ - Chromone $t$-Bu

209g - Chromone c-Hex

209h - Quinoline $t$-Bu

209i - Quinoline c-Hex

209j - Quinoline $t$-Bu

209k - Quinoline c-Hex

209l - Julolidine c-Hex

\section{Scheme 56}


The benzene-1,2-diamine $\mathbf{2 1 0}$ was added to the solution of 2-chloro-6,7-substituted quinoline-3carbaldehydes I in methanol. Ceric ammonium nitrate as a catalyst and hydrogen peroxide as an oxidant were then added to this solution. After completion of the reaction, the reaction mixture was cooled at room temperature and poured into crushed ice. The separated solid product was filtered and recrystallized from methanol to afford 59-79\% yield of The benzo[d] imidazole ligands 211a-g (Scheme 57). ${ }^{77}$

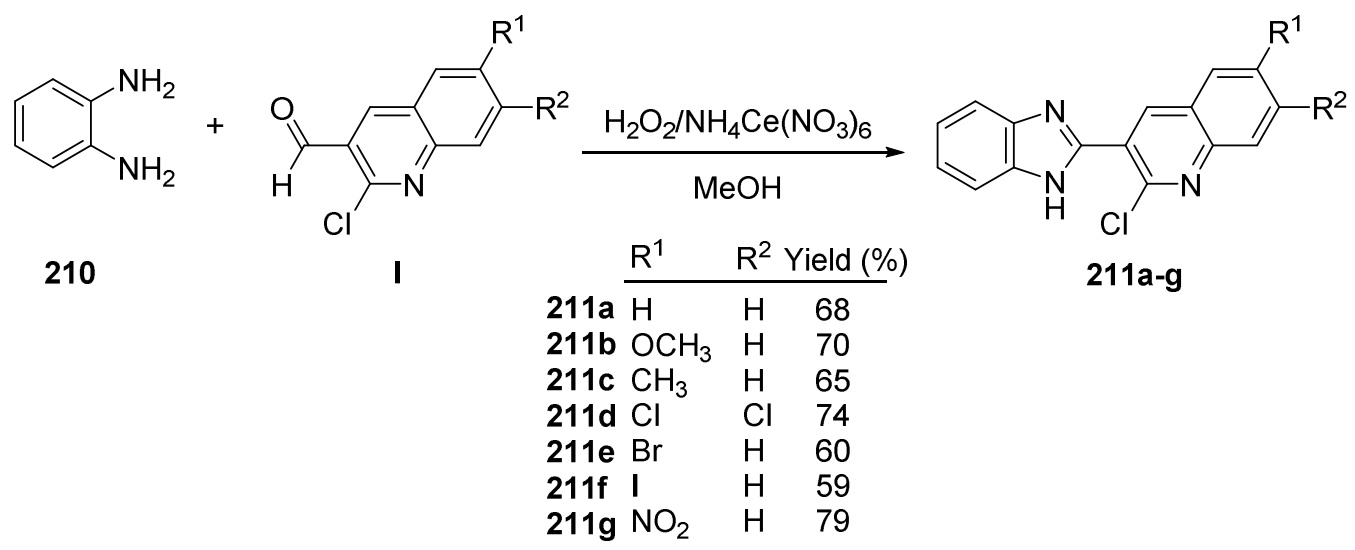

\section{Scheme 57}

An efficient and high yielding protocol is reported for the synthesis of new class of 4-anilinoquinolinoquinazoline hybrids 216a-I, 217a-h. The target compounds were prepared first by the reaction of 2aminobenzamide $\mathbf{2}$ with 2-chloroquinoline-3-carbaldehydes I. After oxidation and chlorination, the key 2quinolyl-4-chloroquinazolines 215a-b were converted to the corresponding 2-(2-arylaminoquinolyl)-4arylaminoquinazolines $\mathbf{2 1 6 a - I}$ and $\mathrm{N}$-heteroaryl-2-(2-(heteroarylamino)quinolin-3-yl)quinazolin-4-amines 217ah (Scheme 58). ${ }^{78}$ 


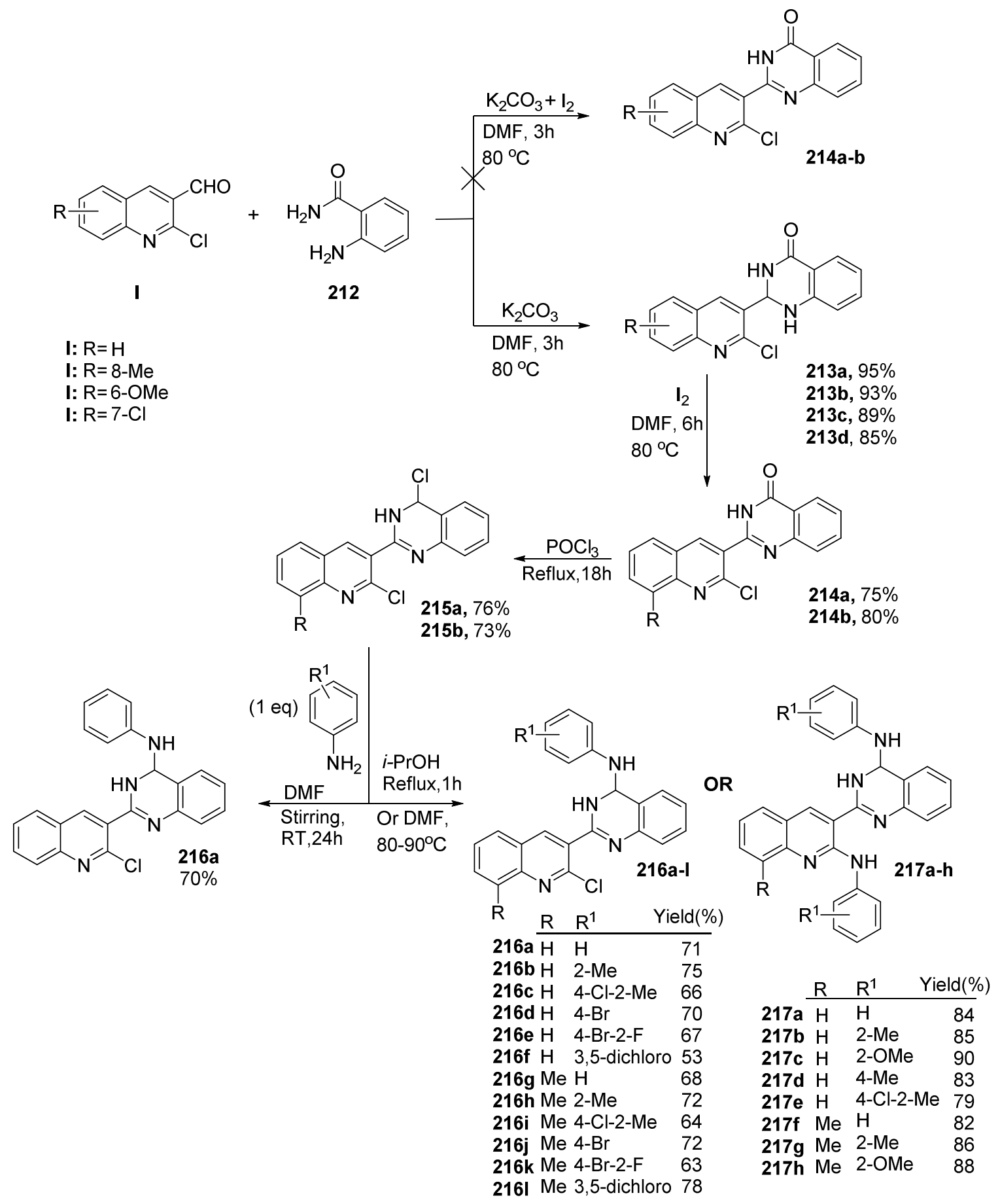

\section{Scheme 58}

3.4.4. Miscellaneous reactions. Condensation of 2-chloroquinoline-3-carbaldehyde I with some selected stabilized phosphonium ylides (Wittig reagent) 218a-d yielded a mixture of the corresponding $E$ and $Z$ olefins in each case along with triphenylphosphine oxide (TPPO), via Wittig carbonyl olefination reaction. However, the reaction of I with $\mathbf{2 1 8 d}$ produced the $(E)$ form olefin $\mathbf{2 1 9 d}$ together with acridin-3-ol $\mathbf{2 2 1}$ (Scheme 59). ${ }^{79}$ 


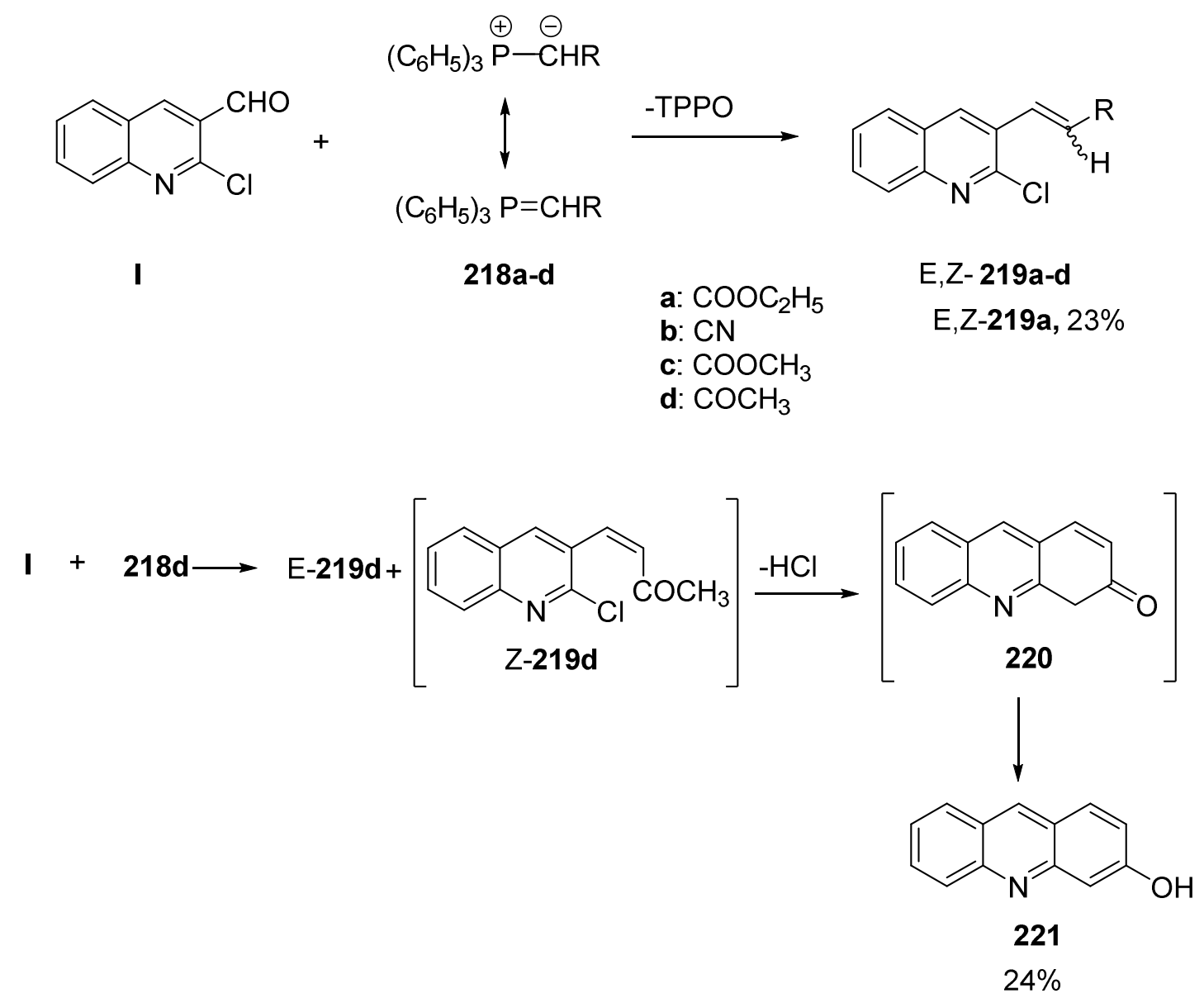

\section{Scheme 59}

A mixture of 2-oxoquinoline-3-carbaldehyde $\mathbf{2 2 2}$ and stabilized ylides $\mathbf{2 1 8} \mathbf{b}$ - $\mathbf{d}$ in absolute ethanol was stirred at room temperature for $2 \mathrm{~h}$. The formed colorless precipitate was filtered and recrystallized from absolute ethanol to give the corresponding Z-223b-d isomers. Pure Z-223b-d were obtained by chromatography (Scheme 60). ${ }^{79}$

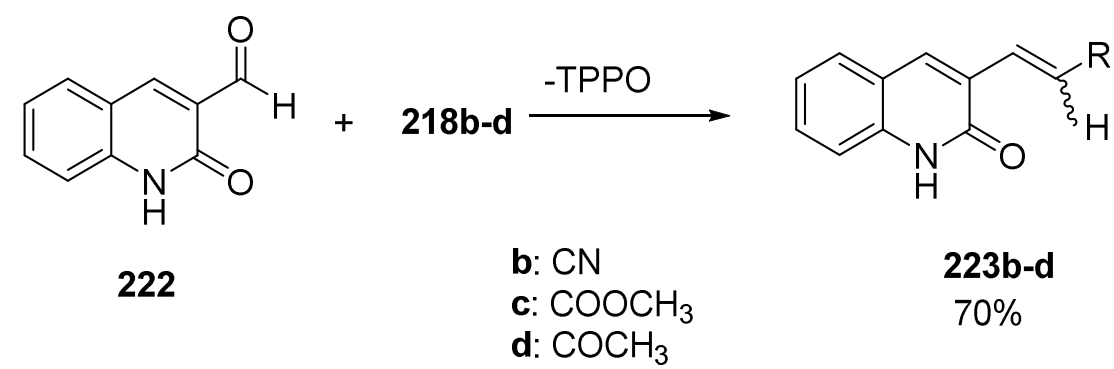

\section{Scheme 60}

The behavior of compounds E,Z-219c toward hydrazine hydrate was also investigated. In which hydrazine hydrate was added to a solution of compound Z-219c and/or E-219c in ethanol. The reaction mixture was heated under reflux for about $3 \mathrm{~h}$. The solvent was evaporated under reduced pressure and the residue was recrystallized from chloroform/n-hexane to give 3-(2-chloroquinolin-3-yl)propanehydrazonic acid 224 (Scheme 61). $^{79}$ 


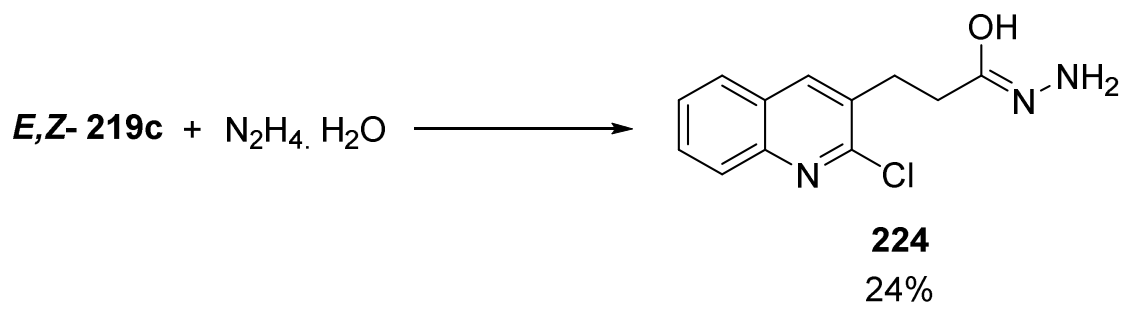

\section{Scheme 61}

On the other hand, heating under reflux a mixture of E-219a or E-219d with the appropriate secondary amine (morpholine $\mathbf{2 2 5 a}$ or piperidine $\mathbf{2 2 5 b}$ ) for 7-10 h, followed by evaporation of the volatile materials and triturating the residue with diethyl ether afforded colorless 226a-d (Scheme 62). ${ }^{79}$

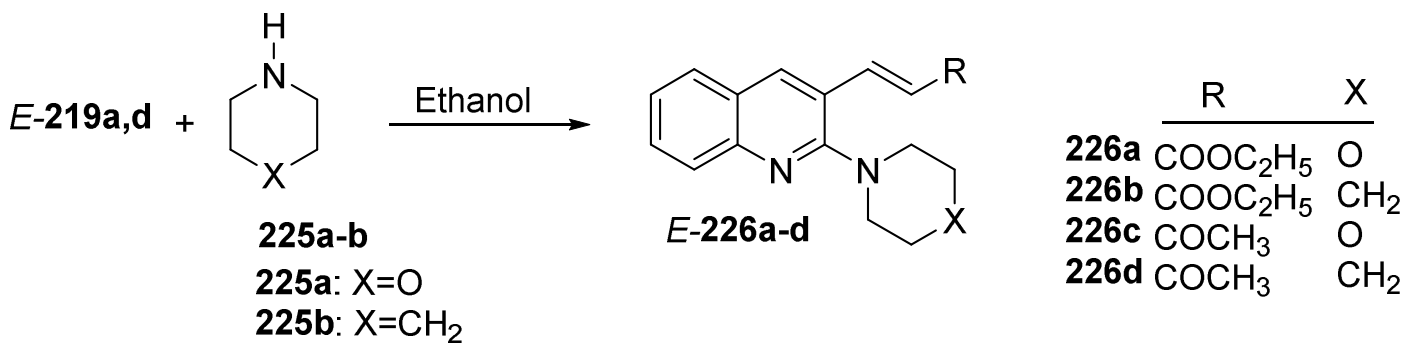

\section{Scheme 62}

Finally, a direct and efficient approach to the synthesis of benzo[ $g][1,8]$ naphthyridines 228a-d, 230a-d from simple synthons 2-chloroquinoline-3-carbaldehydes I, 1 H-Indazole-6-amine 227 and 2-chloroquinolne-4amines 229a-b, has been developed. The reaction proceed by simple condensation under basic medium conditions without any catalyst furnishing the naphthyridine derivatives 228a-d and 230a-d, respectively (Scheme 63)..$^{80}$

$\mathrm{I}: \mathrm{R}=\mathrm{H}$
$\mathrm{I}: \mathrm{R}=6-\mathrm{Me}$
$\mathrm{I}: \mathrm{R}=6-\mathrm{OMe}$
$\mathrm{I}: \mathrm{R}=8-\mathrm{OMe}$
$\mathrm{I}: \mathrm{R}=$ benzo[ $h]$<smiles>O=Cc1cc2cc[R1]cc2nc1Cl</smiles><smiles>[R10]c1ccc2c(N)cc(Cl)nc2c1</smiles><smiles>Nc1ccc2cn[nH]c2c1</smiles>

229a $6-\mathrm{Cl}$ 229b 8-Me<smiles></smiles>

228a-d

\begin{tabular}{ll}
\multicolumn{1}{l}{$\mathrm{R}$} & Yield(\%) \\
228a $\mathrm{H}$ & 60 \\
228b 6-Me & 54 \\
228c 6-OMe & 47 \\
228d benzo[h] & 56
\end{tabular}<smiles>[R16][R1]1ccc2cc3cc4c(Cl)nc5[R]([R])cccc5c4nc3nc2c1</smiles>

\begin{tabular}{lll} 
& \multicolumn{1}{c}{$\mathrm{R}^{1}$} & Yield(\%) \\
\hline 230a 6-Me & $6-\mathrm{Cl}$ & 43 \\
230b 6-OMe & $6-\mathrm{Cl}$ & 40 \\
230c 8-OMe & $6-\mathrm{Cl}$ & 49 \\
230d benzo[h] & 8-Me & 54
\end{tabular}

\section{Scheme 63}




\section{Conclusions}

2-Chloroquinoline-3-carbaldehydes were used extensively, as an interesting versatile intermediate, due to the presence of both chloro and aldehyde groups, for synthesis of many chemical compounds possessing diverse biological activities. This survey is an attempt to collect, summarize and organize the different synthetic methods and reactions of 2-chloroquinoline-3-carbaldehydes from 2012 through 2017. Most of 2chloroquinoline-3-carbaldehydes reactions are multi component reaction (MCR), either in a step-wise manner or in a one pot has been achieved successfully. Hence these protocols provide convenient strategies to annelate different heterocyclic nuclei with widespread bioactive pyrans and pyrimidines thereby extending the categories of heterocyclic systems. The strategies may also provide valuable information for further design and development of more active biological agents through various modifications and derivatizations.

\section{References}

1. Ghanei, S.; Lari, J.; Eshghi, H.; Saadatmandzadeh, M. Iran J. Pharm. Res. 2016, 15, 321.

2. Baruah, B.; Bhuyan, P. J. Tetrahedron 2009, 65, 7099.

https://doi.org/10.1016/j.tet.2009.06.036

3. Rajakumar, P.; Raja, R.; Selvam, S.; Rengasamy, R.; Nagaraj, S. Bioorg. Med. Chem. Lett. 2009, 19 (13), 3466.

https://doi.org/10.1016/i.bmcl.2009.05.019

4. Rajakumar, P.; Raja, R. Tetrahedron Lett. 2010, 51, 4365.

https://doi.org/10.1016/i.tetlet.2010.06.059

5. Domling, A. Chem. Rev. 2006, 106, 17.

https://doi.org/10.1021/cr0505728

6. Radini, I.; Elsheikh, T.; El-Telbani, E.; Khidre, R. Molecules 2016, 21, 909.

https://doi.org/10.3390/molecules21070909

7. Fernández-Galleguillos, C.; Saavedra, L. A.; Gutierrez, M. J. Braz. Chem. Soc. 2014, 25, 365.

8. Salahuddin; M. A.; Shaharyar, M. BioMed. Res. Int. 2014, 2014, Article ID 491492.

9. Musiol, R.; Jampilek, J.; Buchta, V.; Silva, L.; Niedbala, H.; Podeszwa, B.; Palka, A.; Majerz-Maniecka, K.; Oleksyn, B.; Polanski, J. Bioorg. Med. Chem. 2006, 14, 3592.

https://doi.org/10.1016/i.bmc.2006.01.016

10. Rossiter, S.; Péron, J.-M.; Whitfield, P. J.; Jones, K. Bioorg. Med. Chem. Lett. 2005, 15, 4806. https://doi.org/10.1016/i.bmcl.2005.07.044

11. Inoue, M.; Hori, M.; Nakajima, S.; Kitakaze, M.; Fukunami, M.; Ishida, Y.; Abe, H. Arzneimittel-Forschung 1983, 34, 355.

12. Muruganantham, N.; Sivakumar, R.; Anbalagan, N.; Gunasekaran, V.; Leonard, J. T. Biol. Pharm. Bull. 2004, 27, 1683.

https://doi.org/10.1248/bpb.27.1683

13. Marella, A.; Tanwar, O. P.; Saha, R.; Ali, M. R.; Srivastava, S.; Akhter, M.; Shaquiquzzaman, M.; Alam, M. M. Saudi Pharmaceutical Journal 2013, 21 (1), 1-12.

https://doi.org/10.1016/j.jsps.2012.03.002

14. Solomon, V. R.; Lee, H. Curr. Med. Chem. 2011, 18, 1488.

https://doi.org/10.2174/092986711795328382

15. Abdel-Wahab, B. F.; Khidre, R. E. Journal of Chemistry 2013, 2013, 13. 
16. Abdel-Wahab, B. F.; Khidre, R. E., Farahat, A. A.; El-Ahl, A. S., Arkivoc 2012, i, 211. http://dx.doi.org/10.3998/ark.5550190.0010.728

17. Meth-Cohn, O.; Narine, B.; Tarnowski, B., J. Chem. Soc., Perkin Trans 1 1981, 5, 1520. https://doi.org/10.1039/p19810001520

18. Wang, G.-B.; Wang, L.-F.; Li, C.-Z.; Sun, J.; Zhou, G.-M.; Yang, D.-C. Res. Chem. Intermed. 2012, $38,77$. https://doi.org/10.1007/s11164-011-0327-6

19. Gaidhane, M. K.; Ghatole, A. M.; Lanjewar, K. R. Int. J. Pharm. Pharm. Sci. 2013, 5, 421.

20. Romero, A. H. Synth. Commun. 2016, 46, 287.

https://doi.org/10.1080/00397911.2015.1135956

21. Cao, H. T.; Grée, R. Tetrahedron Lett. 2009, 50, 1493. https://doi.org/10.1016/j.tetlet.2009.01.080

22. Zhang, Y. C.; Liu, Z. C.; Yang, R.; Zhang, J. H.; Yan, S. J.; Lin, J. Org. Biomol. Chem. 2013, $11,7276$. https://doi.org/10.1039/c3ob41200h

23. Alam, M. M.; Marella, A.; Akhtar, M.; Husain, A.; Yar, M. S.; Shaquiquzzaman, M.; Tanwar, O. P.; Saha, R.; Khanna, S.; Shafi, S. Acta Pol. Pharm. 2013, 70, 435.

24. Bhanja, C.; Jena, S.; Nayak, S.; Mohapatra, S. Beilstein J. Org. Chem. 2012, 8, 1668. https://doi.org/10.3762/bjoc.8.191

25. Wang, W.; Li, H.; Wang, J.; Zu, L. J. Am. Chem. Soc. 2006, 128, 10354. https://doi.org/10.1021/ja063328m

26. Li, H.; Wang, J.; T, E. N.; Zu, L.; Jiang, W.; Wei, S.; Wang, W. Chem. Commun. 2007, 7, 507. https://doi.org/10.1039/B611502K

27. Govender, T.; Hojabri, L.; Moghaddam, F. M.; Arvidsson, P. I. Tetrahedron: Asymmetry 2006, $17,1763$. https://doi.org/10.1016/i.tetasy.2006.06.028

28. Brohmer MC, V. N., Bräse S. Synlett 2009, 9, 1383.

29. Volz, N; Bröhmer, M C; Toräng, J; Nieger, M; Bräse, S. Indian J. Chem. Sect B. 2009, 48B, 1699.

30. Parmar, N. J.; Labana, B. M.; Barad, H. A.; Kant, R.; Gupta, V. K. Monatsh. Chem. 2014, 145, 1179. https://doi.org/10.1007/s00706-014-1187-8

31. Mistry, B. M.; Jauhari, S. Med. Chem. Res. 2013, 22, 635. https://doi.org/10.1007/s00044-012-0060-8

32. Selva, K.; Subramanian, P. R.; Rayappan, R. Arch. Appl. Sci. Res. 2015, $7,60$.

33. Preveena, N.; Nagendrappa, G.; Kumara, T. S.; Kumar, A. Int. J. Pharm. Sci. Invent. 2015, 4, 53.

34. Fu, L.; Lin, W.; Hu, M.-H.; Liu, X.-C.; Huang, Z.-B.; Shi, D.-Q. ACS Comb. Sci. 2014, 16, 238. https://doi.org/10.1021/co4001524

35. Shiri, M.; Pourabed, R.; Zadsirjan, V.; Sodagar, E. Tetrahedron Lett. 2016, 57, 5435. https://doi.org/10.1016/i.tetlet.2016.10.057

36. Heravi, M. M.; Hosseinnejad, T.; Faghihi, Z.; Shiri, M.; Vazinfard, M. J. Iran. Chem. Soc. 2017, $14,823$. https://doi.org/10.1007/s13738-016-1032-6

37. Ghandi, M.; Zarezadeh, N.; Rahimi, S. J. Iran. Chem. Soc. 2015, 12, 1577. https://doi.org/10.1007/s13738-015-0630-z

38. Kappe, C. O. Eur. J. Med. Chem. 2000, 35, 1043. https://doi.org/10.1016/S0223-5234(00)01189-2

39. Wan, J.-P.; Liu, Y. RSC Advances 2012, 2, 9763. https://doi.org/10.1039/c2ra21406g 
40. Abd-El Maksoud, M. A.; ; Tawfik, H. A.; Maigali, S. S.; Soliman, F. M.; Moharam, M. E.; Dondeti, M. F. Der Pharma Chemica 2016, 8, 291.

41. Dianat, S.; Moghimi, S.; Mahdavi, M.; Nadri, H.; Moradi, A.; Firoozpour, L.; Emami, S.; Mouradzadegun, A.; Shafiee, A.; Foroumadi, A. J. Enzym. Inhib. Med. Chem. 2016, 31, 205. https://doi.org/10.1080/14756366.2016.1206087

42. Kumar, S.; Saunthwal, R. K.; Aggarwal, T.; Kotla, S. K.; Verma, A. K. Org. Biomol. Chem. 2016, $14,9063$. https://doi.org/10.1039/C6OB01539E

43. Ghandi, M.; Zarezadeh, N. Tetrahedron 2013, 69, 8668. https://doi.org/10.1016/i.tet.2013.08.009

44. Ghandi, M.; Zarezadeh, N.; Abbasi, A. Mol. Divers. 2016, 20, 483. https://doi.org/10.1007/s11030-015-9651-x

45. Shiri, M.; Heydari, M.; Zadsirjan, V. Tetrahedron 2017, 73, 2116. https://doi.org/10.1016/j.tet.2017.02.064

46. Ghandi, M.; Zarezadeh, N. J. Iran. Chem. Soc. 2015, 12, 1313. https://doi.org/10.1007/s13738-015-0596-x

47. Ladraa, S.; Chioua, M.; Belfaitah, A. J. Het. Chem. 2017, 54, 603.

48. Rajkumar U. Pokalwar, A. B. C. Chem. Biol. Interface 2013, 3, 339.

49. Tabassum, S.; Kumara, T. S.; Jasinski, J. P.; Millikan, S. P.; Yathirajan, H.; Ganapathy, P. S.; Sowmya, H.; More, S. S.; Nagendrappa, G.; Kaur, M. J. Mol. Struct.2014, 1070, 10. https://doi.org/10.1016/i.molstruc.2014.04.009

50. Ladani, N. K.; Patel, M. P.; Patel, R. G. Arkivoc 2009, vii, 292. http://dx.doi.org/10.3998/ark.5550190.0010.728

51. Kategaonkar, A. H.; Pokalwar, R. U.; Sadaphal, S. A.; Shinde, P. V.; Shingate, B. B.; Shingare, M. S. Heteroat. Chem. 2009, 20, 436.

52. Deshmukh, A. R.; Bhosle, M. R.; Khillare, L. D.; Dhumal, S. T.; Mishra, A.; Srivastava, A. K.; Mane, R. A. Res.Chem. Intermed. 2017, 43, 1107. https://doi.org/10.1007/s11164-016-2686-5

53. Rao, K. R.; Bhanumathi, N.; Sattur, P. B. J. Het. Chem. 1991, 28, 1339.

54. Hamama, W. S.; Ibrahim, M. E.; Gooda, A. A.; Zoorob, H. H. Synth. Commun. 2017, 47, 224. https://doi.org/10.1080/00397911.2016.1258580

55. Ramesh, V.; Ananda Rao, B.; Sharma, P.; Swarna, B.; Thummuri, D.; Srinivas, K.; Naidu, V. G.; Jayathirtha Rao, V. Eur. J. Med. Chem. 2014, 83, 569.

https://doi.org/10.1016/i.ejmech.2014.06.013

56. Hussain, S.; Jadhav, S.; Rai, M.; Farooqui, M. International Journal of Pharmaceutical, Chemical \& Biological Sciences 2014, 4, 126.

57. Shelke, K. F.; Khadse, R. E. Der Pharma Chemica, 2015, 7, 191.

58. Mirjafary, Z.; Saidian, H.; Sahandi, M.; Shojaei, L. J. Braz. Chem. Soc. 2014, 25, 1253.

59. Fu, L.; Feng, X.; Wang, J.-J.; Xun, Z.; Hu, J.-D.; Zhang, J.-J.; Zhao, Y.-W.; Huang, Z.-B.; Shi, D.-Q. ACS Com. Sci. 2014, 17, 24.

60. Shiri, M.; Nejatinezhad-Arani, A.; Faghihi, Z. J. Het. Chem. 2017, 54, 131.

61. Paplal, B.; Nagaraju, S.; Veerabhadraiah, P.; Sujatha, K.; Kanvah, S.; Kumar, B. V.; Kashinath, D. RSC Advances 2014, 4, 54168.

https://doi.org/10.1039/C4RA07708C 
62. Marco-Contelles, J.; Pérez-Mayoral, E.; Samadi, A.; Carreiras, M. d. C.; Soriano, E. Chem. Rev. 2009, 109, 2652.

https://doi.org/10.1021/cr800482c

63. Garcia-Font, N.; Hayour, H.; Belfaitah, A.; Pedraz, J.; Moraleda, I.; Iriepa, I.; Bouraiou, A.; Chioua, M.; Marco-Contelles, J.; Oset-Gasque, M. J. Eur. J. Med. Chem. 2016, 118, 178.

https://doi.org/10.1016/j.ejmech.2016.04.023

64. Joshi, S. D.; More, U. A.; Parkale, D.; Aminabhavi, T. M.; Gadad, A. K.; Nadagouda, M. N.; Jawarkar, R. Med. Chem. Res. 2015, 24, 3892.

https://doi.org/10.1007/s00044-015-1432-7

65. Ghanei, S.; Eshghi, H.; Lari, J.; Saadatmandzadeh, M. Journal of Chemical and Pharmaceutical Research 2015, 7, 428.

66. Chandrika, N. K., T. H. Suresha; Nagendrappa, Gopalpur; Chetana, P. R.; Sowmya, H. B. V.; Rashmi, S. K.;Dileep, R.; Sandeep, C. J. Applicable. Chem. 2013, 2, 1535.

67. Kumara, T. H. S.; Nagandrappa, G.; Chandrika, N.; Sowmya, H. B.; Kaur, M.; Jasinski, J. P.; Glidewell, C. Acta Crystallogr. Sec. C 2016, 72, 670.

https://doi.org/10.1107/S205322961601278X

68. Salahuddin, S.; Mazumder, A.; Shaharyar, M. Med. Chem. Res. 2015, 24, 2514.

https://doi.org/10.1007/s00044-014-1308-2

69. Jha, K. K.; Samad, A.; Kumar, Y.; Shaharyar, M.; Khosa, R. L.; Jain, J.; Kumar, V.; Singh, P. Eur. J. Med. Chem. 2010, 45, 4963.

https://doi.org/10.1016/i.ejmech.2010.08.003

70. Alexander, E. R.; Wildman, R. B. J. Am. Chem. Soc. 1948, 70, 1187.

https://doi.org/10.1021/ja01183a091

71. Nasielski-Hinkens, R.; Levêque, P.; Castelet, D.; Nasielski, J. Heterocycles 1987, 26, 2443. https://doi.org/10.3987/R-1987-09-2433

72. Torrini, I.; Zecchini, G. P.; Paradisi, M. P.; Francesca, S. Heterocycles 1990, 31, 1687. https://doi.org/10.3987/COM-90-5492

73. Shinde, A. D.; Sonar, S. S.; Shingate, B. B.; Shingare, M. S. Phosphorus Sulfur Silicon Relat. Elem. 2010, 185, 1594.

https://doi.org/10.1080/10426500903136913

74. Hamidi, H.; Heravi, M. M.; Tajbakhsh, M.; Shiri, M.; Oskooie, H. A.; Shintre, S. A.; Koorbanally, N. A. J. Iran. Chem. Soc. 2015, 12, 2205.

https://doi.org/10.1007/s13738-015-0698-5

75. Karimian, A.; Eshghi, H.; Bakavoli, M.; Shiri, A. Heterocycl. Commun. 2014, 20, 275.

https://doi.org/10.1515/hc-2014-0095

76. Kishore, K. G.; Islas-Jácome, A.; Rentería-Gómez, A.; Conejo, A. S.; Basavanag, U. M. V.; Wrobel, K.; Gámez-Montaño, R. Tetrahedron Lett. 2016, 57, 3556.

https://doi.org/10.1016/i.tetlet.2016.06.120

77. Maru, M. S.; Shah, M. K. Orbital: Electron. J. Chem. 2015, 7, 108.

78. Derabli, C.; Boulcina, R.; Kirsch, G.; Debache, A. Tetrahedron 2017, 73, 351.

https://doi.org/10.1016/j.tet.2016.12.009

79. El-Samahy, F. A.; Ibrahim, N. M.; Mahran, M. R. H. Phosphorus Sulfur Silicon Relat. Elem. 2016, $191,59$. https://doi.org/10.1080/10426507.2015.1085042 
80. Magesh Selva Kumar, A. M.; Vijaya Pandiyan, B.; Mohana Roopan, S.; Rajendran, S. P., J.Photochem.Photobiol. A 2017, 332, 72.

https://doi.org/10.1016/i.jphotochem.2016.08.014

\section{Authors Biographies}

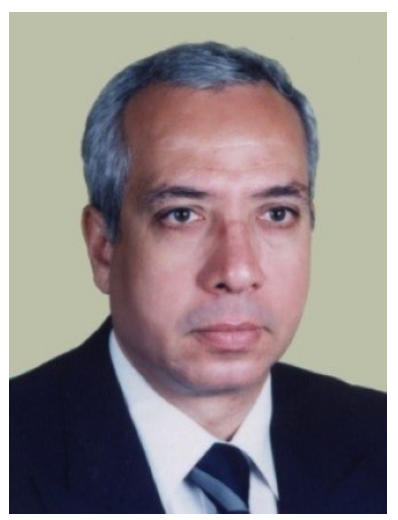

Mohamed A. M. Massoud was born in 1949 in Cairo, Egypt. Now he is a professor emeritus in Pharmaceutical Organic Chemistry Department, Faculty of Pharmacy, Mansoura University, Mansoura, Egypt. He received his M.Sc. in 1975 from Pharmaceutical Organic Chemistry Department, Faculty of Pharmacy, Cairo University. He has awarded his Doctor degree, under supervision of Professor J. M. J. Tronchet, in 1982 from Faculty of Pharmacy, Geneva University, Switzerland in the synthesis of Lincomycin analogues. Since 1982, he has been a lecturer at the University of Mansoura, Egypt in the Faculty of Pharmacy, Mansoura University, Mansoura, Egypt. Then graduated until occupied the Chairman, Department of Pharmaceutical Organic Chemistry, University of Mansoura, Egypt (2003-2008). In addition, he acted as professor visitor and external examiner in several Egyptian and Arabic Universities. His research interest is the synthesis of new biologically active heterocyclic compounds with pharmaceutical interests.

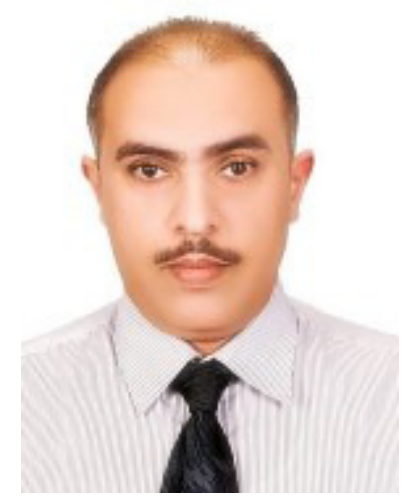

Waleed A. Bayoumi was born in 1972 in Mansoura, Egypt. In 1995, He graduated from Faculty of Pharmacy, Mansoura University, Egypt. He obtained his M.Sc. in Pharmaceutical Organic Chemistry in 2001 from Medicinal Chemistry Department, Faculty of Pharmacy, Mansoura University, Egypt. He was awarded his Ph.D. in Pharmaceutical Organic Chemistry in 2007 from Faculty of Pharmacy, Mansoura University. He teaches the courses of Pharmaceutical Organic Chemistry in several Universities. His main research area is the design and synthesis of heterocyclic compounds of pharmaceutical interests. He also has interests in the fields of elearning and quality assurance in education. 


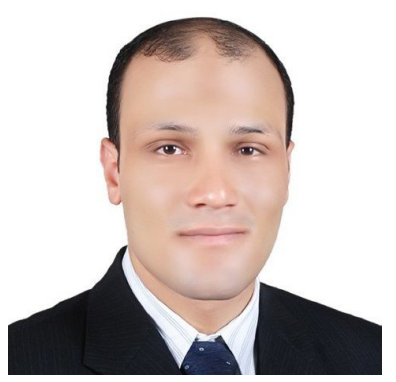

Abdelbasset A. Farahat: was born in 1980 in Mansoura, Egypt. He is a research scientist of Medicinal Chemistry at Georgia State University, Atlanta, Georgia, USA. He received his B.Sc. in 2002 from Faculty of Pharmacy, Mansoura University, Egypt. He received his M.Sc. in 2006 from Pharmaceutical Organic Chemistry Department, Faculty of Pharmacy, Mansoura University, Egypt. He has awarded his Ph.D. degree in 2010 after a joint program between Mansoura University and Georgia State University, Atlanta, Georgia, USA, under the supervision of Professor David W. Boykin. He is working now on A Gates and NIH funded projects titled" Drug discovery for parasitic diseases" and "Synthesis of G-Recognition Units for DNA Minor-groove Recognition".

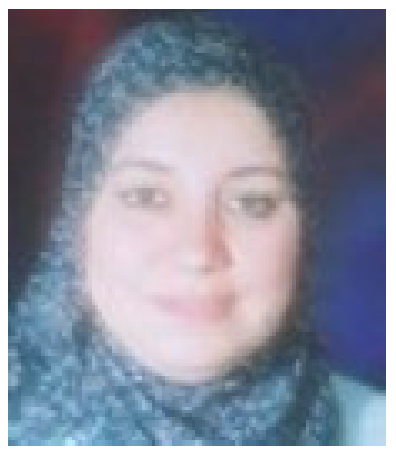

Magda Abdel-Aziz El-Sayed was born in 1972 in Mansoura, Egypt. She has got her B.Sc. in 1995 from Faculty of Pharmacy, Mansoura University, Egypt. She received her M.Sc. in 2001 from Pharmaceutical Organic Chemistry Department, Faculty of Pharmacy, Mansoura University, under the supervision of Professor Ali A. ElEmam. She performed her thesis on model studies for synthesis of certain 6-(arylthio)uracils and related derivatives as potential antiviral agents. She has awarded her Ph.D. degree in 2007 under the supervision of Professor Mohamed A. M. Massoud. She performed her Ph.D. thesis on model studies for synthesis and biological evaluation of new unsaturated derivatives of cyclic compounds as potent antioxidant agent. Her research interest is the design and synthesis of heterocyclic compounds with pharmaceutical interests.

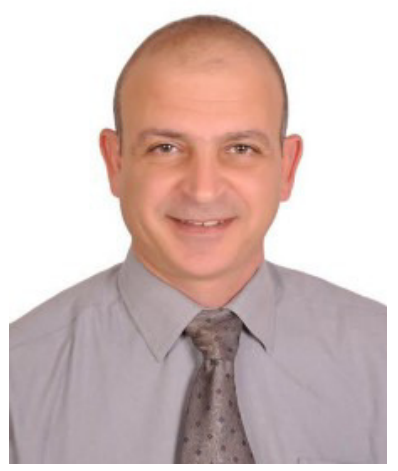

Basem A. Mansour was born in 1973, in Dikirness, Daqahlya, Egypt. He is an assistant lecturer of pharmaceutical organic chemistry, faculty of pharmacy, Delta University for science and technology, Gamasa, 
Egypt. He has got his B.Sc. in May 1996 from faculty pharmacy, Mansoura University, Egypt. Conferred his Master degree in Pharmaceutical Organic Chemistry at the same college in March 2014. Master thesis was entitled "Design and synthesis of novel benzimidazoles of potential anthelmintic activity". Enrolled to PhD program since 2014 through now at the same department he had granted his master from. PhD thesis entitled "Synthesis of certain Quinoline derivatives as antitumor agents". 